\title{
DEVELOPMENT AND CHARACTERIZATION OF REAGENT PENCILS FOR MICROFLUIDIC PAPER BASED ANALYTICAL DEVICES
}

\author{
A Thesis \\ presented to \\ the Faculty of California Polytechnic State University, \\ San Luis Obispo

\begin{abstract}
In Partial Fulfillment
of the Requirements for the Degree
\end{abstract} \\ Master of Science in Polymers and Coatings Science
}

by

Cheyenne H. Liu

June 2016 
(C) 2016

Cheyenne H. Liu

ALL RIGHTS RESERVED 
TITLE:

AUTHOR:

DATE SUBMITTED:

COMMITTEE CHAIR:

COMMITTEE MEMBER:

COMMITTEE MEMBER:
Development and Characterization of Reagent Pencils for Microfluidic Paper Based Analytical Devices Cheyenne H. Liu June 2016

Philip J. Costanzo, Ph.D. Associate Professor of Chemistry and Biochemistry Andres W. Martinez, Ph.D. Assistant Professor of Chemistry and Biochemistry Chad Immoos, Ph.D. Professor of Chemistry and Biochemistry 


\begin{abstract}
Development and Characterization of Reagent Pencils for Microfluidic Paper Based Analytical Devices

Cheyenne H. Liu
\end{abstract}

Microfluidic paper based analytical devices (microPADs) are a novel platform for point of care (POC) diagnostics. Limitations of reagent shelf life have been overcome with the introduction of reagent pencils as a method for solid-based reagent deposition. While useful, little work has been reported on the characterization and optimization of reagent pencils. Herein, an investigation on reagent pencil composition and efficiency is conducted via colorimetric release profile tests utilizing an erioglaucine disodium salt that yields a quantifiable blue colored product in the presence of water. Within this work, an investigation on the molecular weight dependence, polymer chain end functionality, and polymer-graphite ratio was conducted to determine the most desirable parameters in reagent pencil composition. Further, the effects of enzyme stability in the presence of poly(ethylene glycol) (PEG) is investigated.

To show the versatility of reagent pencils, a novel reagent pencil incorporating a stimuli responsive polymer, poly(N-isporopylacrylamide) (PNIPAM) was developed. In this work, PNIPAM's lower critical solution temperature (LCST) was manipulated with various salt solutions to control fluid flow both laterally and vertically through various microPAD designs. It was found that, while PNIPAM successfully blocked or retarded fluid 
flow in microPADs, the effect was limited when $\mathrm{DI}_{2} \mathrm{O}$ wash solutions were run prior to salt solutions. To counteract this, PNIPAM was successfully covalently bound to alkene modified chromatography paper via thiolene click chemistry to reinforce solution wash tolerance. 


\section{ACKNOWLEDGMENTS}

I would like to thank members of my committee for their guidance not only throughout this project, but also for their guidance throughout my time at Cal Poly.

In particular, I would like to thank Dr. Philip Costanzo for your unwavering support and, for lack of better description, gung-ho attitude. I can say with absolute confidence that your particular mindset of going all-in has motivated and benefited all students who have had the privilege of working with you in aspects beyond simply academia. Thank

you especially to Dr. Andres Martinez and Dr. Chad Immoos for allowing me the opportunity to work in your labs in an open, relaxed environment. Both of your guidance throughout my time at Cal Poly has pushed me to be successful in my work.

Finally, I would like to thank my past and present confidantes in lab - Belle Noxon, Haydn Mitchell, Daisy Jauregui, Kirsten Ganaja, Kyle Chan, and Cody Yokubaitis. Your collective presence in lab has kept me humble, sane, and productive. Most importantly, working in lab with this specific group has inspired the next step in my career and refined my taste in music.

Without a doubt, all of the people aforementioned and many of whom could not possibly fit on this page have allowed me to grow as a person, and I will be leaving Cal Poly undoubtedly as a better worker, scientist, and human being. Thank you! 


\section{TABLE OF CONTENTS}

Page

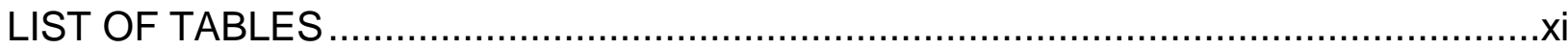

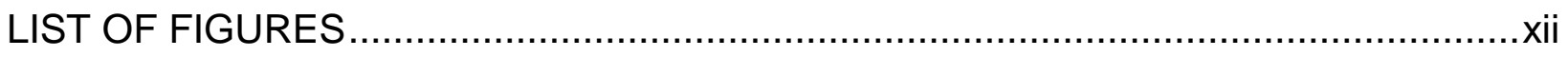

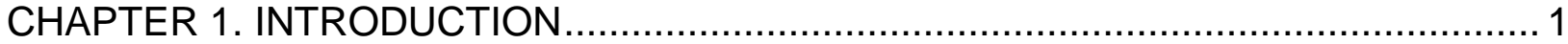

1.1. Microfluidic Paper-based Analytical Devices ................................................. 1

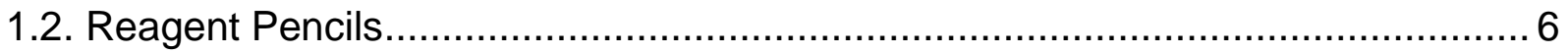

1.3. Horseradish Peroxidase (HRP) Structure, Function and Applications ................. 9

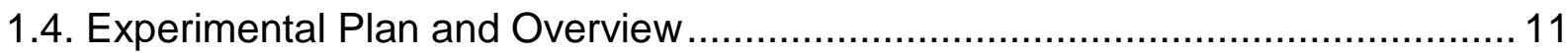

CHAPTER 2. CHARACTERIZATION OF POLYMER COMPOSITION, REAGENT TYPE, AND RELEASE EFFICIENTY FOR REAGENT PENCILS ............................. 13

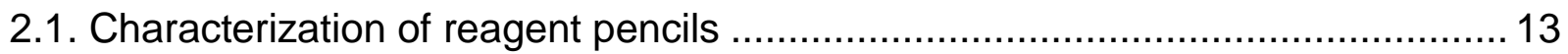

2.1.1. Characterization of PEG and PEG derivative reagent pencil systems ........ 13

2.1.2. Characterization of erioglaucine disodium salt reagent pencils ................... 16

2.1.3. Colorimetric release profile for blue dye reagent pencil systems ................. 19

2.1.4. Characterization of blue dye, HRP, and glucose PEGdiME reagent pencils

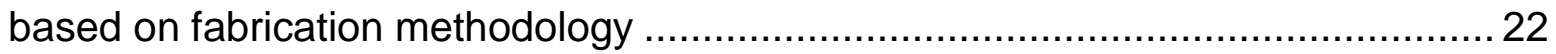

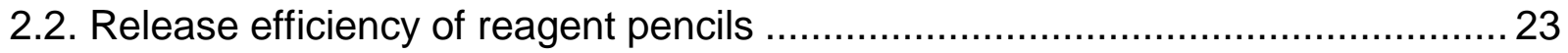

2.3. Solution and Solid Polymer Stabilization of HRP and ALP .............................. 28

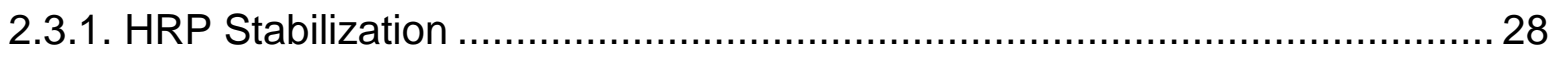

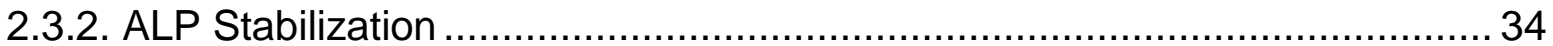

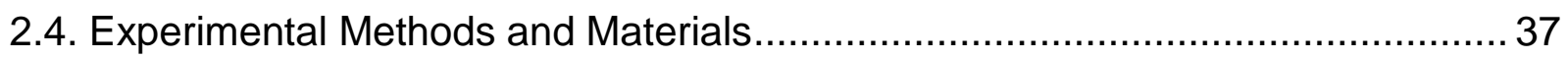

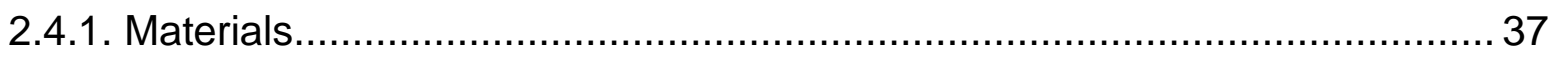

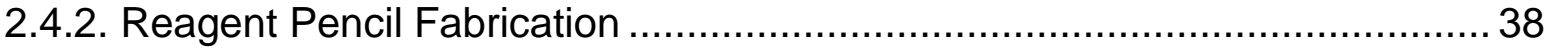

2.4.2.1. Fabrication of reagent pencils via rotary evaporation .......................... 38

2.4.2.2. Fabrication of reagent pencils via lyophilization................................... 39

2.4.3. Fabrication of paper-based devices ........................................................ 39 
2.4.4. Differential Scanning Calorimetry

2.4.5. Determination of wear for PEG-graphite systems at varying pellet diameters 41

2.4.6. Characterization of blue dye PEG-graphite colorimetric release profile ....... 42

2.4.7. Solution deposition calibration.............................................................. 43

2.4.7.1. Solution deposition calibration of blue dye ........................................ 43

2.4.7.2. Solution deposition calibration of glucose ........................................ 43

2.4.7.3. Solution deposition calibration of horseradish peroxidase ................... 44

2.4.8. Reagent Pencil Release Efficiency ....................................................... 44

2.4.8.1. Blue dye and glucose reagent pencil efficiency ................................. 44

2.4.8.2. Horseradish peroxidase reagent pencil efficiency. .............................. 45

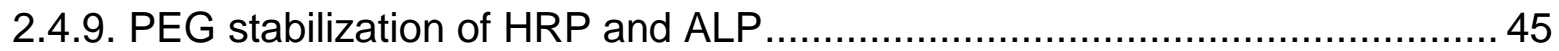

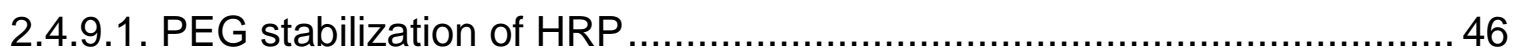

2.4.9.2. PEG stabilization of ALP................................................................... 47

CHAPTER 3. MANIPULATION OF PNIPAM LCST FOR FLUID FLOW

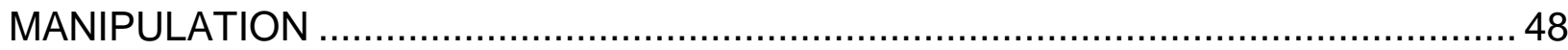

3.1. Thermoresponsive Polymers and Click Chemistry ....................................... 48

3.1.1. Characteristics of Thermoresponsive Polymers and Poly $(\mathrm{N}-$

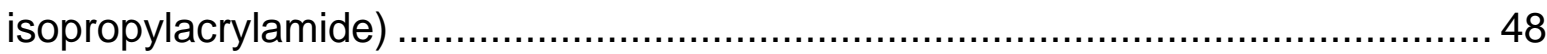

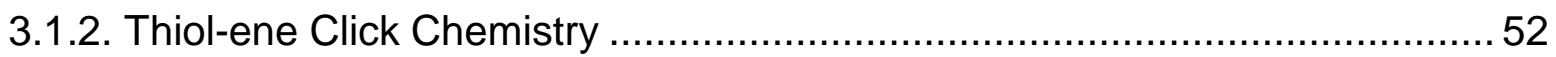

3.1.3. Experimental Plan and Overview .......................................................... 53

3.2. Characterization of PEG/PNIPAM Reagent Pencil Systems ............................ 55

3.2.1. Effect of graphite variance and polymer on wear, colorimetric release

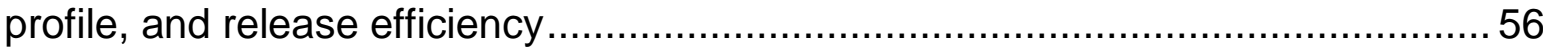

3.2.2. Effect of PEG on PNIPAM for wear, colorimetric release profile, and release efficiency 59 
3.3. Use of PNIPAM as a stimuli responsive tool for microPADs............................. 61

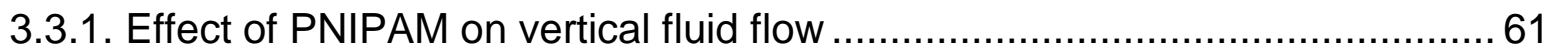

3.3.1.1. Determination of salt effects on PNIPAM LCST for simple vertical

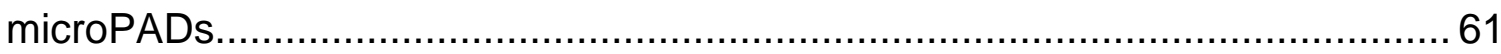

3.3.1.2. Manipulation of PNIPAM LCST in complex vertical flow microPADs ..... 64

3.3.1.3. Contact angle studies for complex vertical flow microPAD ....................66 66

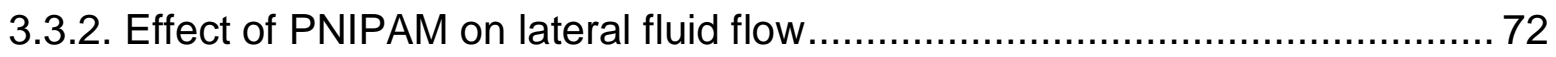

3.4. Reagent Condition tolerance in preparation for photoinitiated click chemistry .... 78

3.4.1. Solvent and UV irradiation tolerance of HRP ............................................ 79

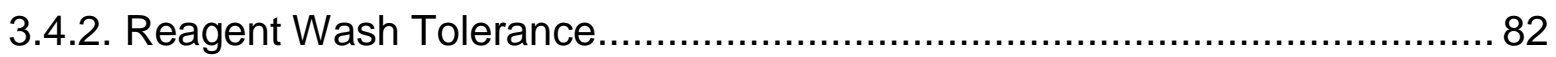

3.4.2.1. Reagent Lateral Flow Wash Tolerance............................................ 82

3.4.2.2. Reagent Vertical Flow Wash Tolerance........................................... 85

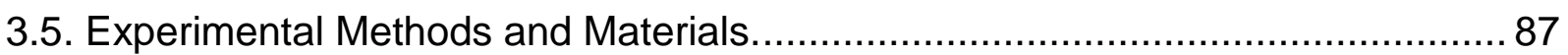

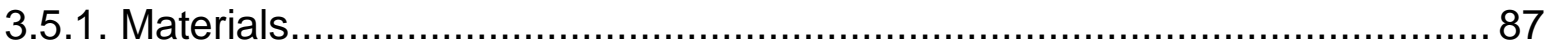

3.5.2. PNIPAM Synthesis of dithiobenzene and nitrile terminated PNIPAM.......... 87

3.5.2.1. Synthesis of carboxylic acid terminated PNIPAM ................................. 87

3.5.2.2. Synthesis of thiol terminated PNIPAM .............................................. 87

3.5.3. Manipulation of fluid flow via PNIPAM LCST alteration.............................. 88

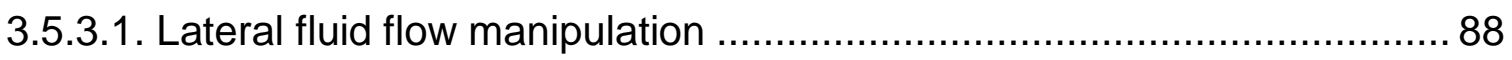

3.5.3.1.1. PNIPAM lateral flow wash tolerance ............................................. 89

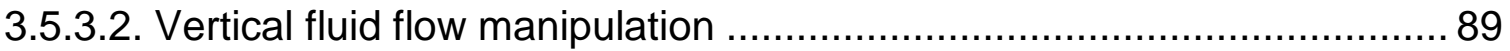

3.5.3.3. Contact angle studies on complex vertical flow microPADs ................. 91

3.5.4. Fabrication of alkene-functionalized chromatography paper....................... 91

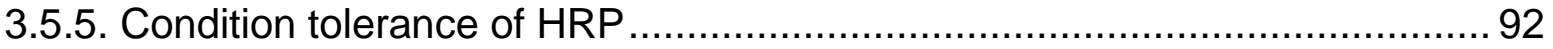

3.5.5.1. DMPA/DMF tolerance of HRP via solution deposition ......................... 92

3.5.5.2. UV tolerance of HRP via solution deposition ...................................... 92 
3.5.5.3. DMPA/DMF and UV tolerance of HRP via solution deposition ............. 92

3.5.6. A novel microPAD for HRP immobilization via thiolene click chemistry ....... 93

CHAPTER 4. CONCLUSIONS AND FUTURE WORK ............................................. 94

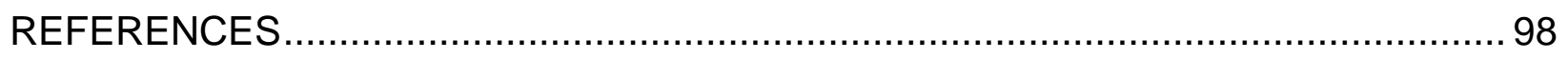




\section{LIST OF TABLES}

Table

Page

Table 1. Tm of PEG-graphite systems via DSC. …................................................... 14

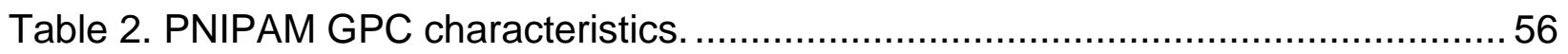




\section{LIST OF FIGURES}

Figure

Figure 1. Fabrication of reagent pencils via photolithography or wax printing ${ }^{11}$............ 3

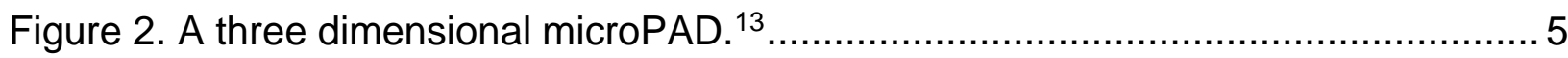

Figure 3. Shelf life of (A) HRP and (B) ALP in different formats, including pencil

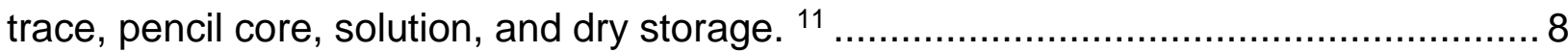

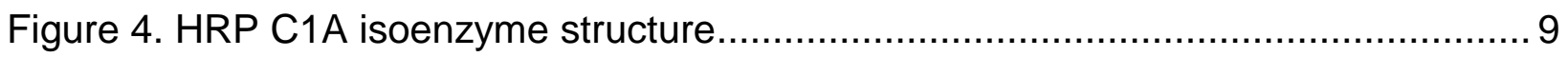

Figure 5. Conversion of ABTS in the presence of $\mathrm{H}_{2} \mathrm{O}_{2}$ by HRP yields a radical product that appears green and absorbs at $410 \mathrm{~nm}$ and $650 \mathrm{~nm}$.

Figure 6. Comparison of PEG-graphite pencil deposition at different diameters under a microscope where (A) PEG 2000, $6.35 \mathrm{~mm}$ visibly deposits less reagent than (B) PEG 2000, $3.00 \mathrm{~mm}$ and (C) PEG 6000, $3.00 \mathrm{~mm}$ reagent pencils.

Figure 7. Wear comparison of PEG-graphite pellets at varying compositions and pellet diameters.

Figure 8. Investigation of PEG functionalization as PEGME and PEGdiME on wear both with and without $15 \% \mathrm{w} / \mathrm{w}$ blue dye.

Figure 9. Wear measurements for PEG 2000, PEGdiME 2000, and PEG 6000 pencil cores with varying blue dye concentrations.

Figure 10. Comparison of 3mm 15\% blue dye PEG-graphite pencil core formulations via a colorimetric release profile test on microPADs.

Figure 11. Release profile signal intensities for PEG 2000, PEGdiME 2000, and PEG 6000 pencil cores with varying blue dye concentrations

Figure 12. Comparison of wear for PEGdiME rotary evaporated and lyophilized pencil cores with $5 \% \mathrm{w} / \mathrm{w}$ of blue dye, glucose, or horseradish peroxidase.

Figure 13. A) Colorimetric release profile and its B) reciprocal release profile for blue dye via solution deposition at known concentrations. Signal intensity for respective 
reagent pencils containing $10,7.5$, or $5 \%$ blue dye are compared release profile curve.

Figure 14. A) Colorimetric release profile and its B) reciprocal release profile for horseradish peroxidase via solution deposition at known concentrations on a 96 well microPAD

Figure 15. Colorimetric release profile for glucose via solution deposition at known concentrations 26

Figure 16. Efficiency of blue dye and glucose reagent pencils with PEGdiME 2000 as polymer component of pencil core.

Figure 17. Signal intensity of PEG and PEGdiME reagent pencils with varying glucose concentration.

Figure 18. Comparison of HRP activity stabilization in the presence of solid or solution based deposited PEG.

Figure 19. HRP activity in the presence of $A$ ) equivalent mass delivery of PEG and $B$ ) equivalent concentration of solution deposited PEG at varying molecular weights. 32 Figure 20. HRP activity in the presence of solution deposited (A) PEG 2000, (B) PEG 600, (C) PEG 400, and (D) PEG 200 at varying polymer concentrations 33 Figure 21. Comparison of ALP (A) wash tolerance and (B) PEG stabilization effects with either solution or solid deposition.

Figure 22. Fabrication of reagent pencils via a manual pellet press to create a 3 or $6.35 \mathrm{~mm}$ diameter pencil core.

Figure 23. Depiction of (A) simple and B) complex lateral flow device designs used for release profile testing.

Figure 24. Depiction of $(A)$ simple and $(B)$ complex vertical flow device designs.

Figure 25. Depiction of wear resistance testing. Pencil cores were loaded onto a pen plotter and deposited reagent at a constant velocity, load, and distance. 
Figure 26. Phase diagram for a polymer blend that has both a UCST and LCST. The Coexistence curve is also known as the binodal curve. ${ }^{16}$ 50

Figure 27. PNIPAM conformation from a molecular dynamic simulation at (A) $295 \mathrm{~K}$ and $(B) 310 K^{23}$

Figure 28. Scheme of chromatography paper functionalization via photoinitiated thiolene click chemistry.

Figure 29. GPC traces and corresponding structure for (A) nitrile and dithiobenzene terminated PNIPAM and (B) thiol terminated PNIPAM. 56

Figure 30. pNIPAM vs PEG variants in wear. no BD incorporated. 58

Figure 31. Trends in $(A)$ wear and $(B)$ release profile for varying graphite concentration at constant w/w 5 wt\% blue dye in PEGdiME 2000 and pNIPAM. (C) shows the release efficiency of pNIPAM samples with varying graphite concentration. 58 Figure 32. Trends in (A) wear, (B) release profile and (C) release efficiency for pNIPAM-PEG 2000 systems with $5 \% \mathrm{w} / \mathrm{w}$ blue dye. 60

Figure 33. Effect of salt composition and concentration on $0.125 \mathrm{mM} \mathrm{BD}$ signal on a 96 well plate with pre-deposited PNIPAM-graphite.

Figure 34. Effect of $\mathrm{Na2SO} 4$ and $\mathrm{NaCl}$ concentration on $0.125 \mathrm{mM} \mathrm{BD}$ signal on a 96 well plate with pre-deposited PNIPAM-graphite. 63 Figure 35. Effect of $\mathrm{Na2SO} 4$ concentration on $0.125 \mathrm{mM}$ BD signal on a 96 well plate with pre-deposited PNIPAM-graphite or PNIPAM solution. (PNIPAM solutions in DMF; all used PNIPAM-graphite DI wash control) 64 Figure 36. Complex vertical microPAD with various amount of PNIPAM doping 66 Figure 37. Contact angle measurements over time for vertical flow microPAD with 4 layers. (A) DI wash only; (B) Layer A with 26mM 4k PNIPAM and $1 \mathrm{M} \mathrm{Na}_{2} \mathrm{SO}_{4}$; (C) Layer A, B, C with 26mM 4k PNIPAM and $1 \mathrm{M} \mathrm{Na}_{2} \mathrm{SO}_{4}$ 68 
Figure 38. Contact angle measurements over a minute for (A) $9 \mathrm{mM}$ 16k PNIPAM, (B) $6 \mathrm{mM}$ 16k PNIPAM, (C) $26 \mathrm{mM} 4 \mathrm{k}$ PNIPAM with a $1 \mathrm{M} \mathrm{Na}_{2} \mathrm{SO}_{4}$ wash, and (D) DI solution with no PNIPAM. All PNIPAM samples were deposited onto Layer A.

Figure 39. Contact angle vs time for varying concentrations and molecular weights of PNIPAM with either $1 \mathrm{M} \mathrm{Na} 2 \mathrm{SO}_{4}$ or $\mathrm{DI} \mathrm{H}_{2} \mathrm{O}$ washes over (A) 60 seconds and (B) 30 seconds

Figure 40. Salt and PNIPAM concentration variance on BD fluid flow. 73

Figure 41. Solution and solid deposition of PNIPAM on lateral flow devices with $0.125 \mathrm{mM} \mathrm{BD}$ only $\left(A\right.$ and $\mathrm{D}$ ), with $1 \mathrm{M} \mathrm{Na}_{2} \mathrm{SO}_{4}$ and $0.125 \mathrm{mM} \mathrm{BD}$ ( $B$ and $E$ ), and with $4 \times 13 \mu \mathrm{l} \mathrm{DI} \mathrm{H}_{2} \mathrm{O}$ wash prior to $1 \mathrm{M} \mathrm{Na}_{2} \mathrm{SO}_{4}$ and $0.125 \mathrm{mM} \mathrm{BD}$ addition ( $\mathrm{C}$ and $\mathrm{F}$ ) 74

Figure 42. PNIPAM (A) solid and (B) solution deposition of 4k PNIPAM wash tolerance up to 5 times the device volume. After washing, $13 \mu \mathrm{l}$ of $1 \mathrm{M} \mathrm{Na}_{2} \mathrm{SO}_{4}$ and $0.125 \mathrm{mM}$ BD was pipetted into the sample addition zone.

Figure 43. IR spectroscopy of unmodified, alkene functionalized, and PNIPAM functionalized chromatography paper. 75 Figure 44. Comparison of $(A) 4 k$ and $(B, C)$ 16k PNIPAM efficiency when clicked onto alkene functionalized paper and not clicked on unmodified paper.

Figure 45. Comparison of HRP condition tolerance to (A) UV exposure time, (B) solvent/reagent order of addition, and (C) DMF and UV tolerance at varying concentrations of $\operatorname{HRP}(20,10,5,3,1,0.5$, and $0.1 \mathrm{U} / \mathrm{mL} \mathrm{HRP})$. 81

Figure 46. (A) Caterpillar lateral flow device used to determine reagent wash tolerance. The response profiles of (B) HRP and (C) $0.125 \mathrm{mM} \mathrm{BD}$ are shown. 84 Figure 47. Vertical flow wash tolerance of (A) $0.125 \mathrm{mM} \mathrm{BD}$ and (B) $20 \mathrm{U} / \mathrm{mL}$ HRP. ... 86 Figure 48. Novel microPAD design for HRP immobilization via thiol-ene click chemistry. 


\section{CHAPTER 1. INTRODUCTION}

\subsection{Microfluidic Paper-based Analytical Devices}

Paper-based diagnostic tests have been developed extensively for applications in both clinical and analytical chemistry because of their accessibility, versatility and simplicity. The need for paper-based diagnostic tests stems from the demand for simple and accurate tests that can be performed on-site or at the point of care. Ideally, paperbased diagnostic tests can become time efficient and cost effective tools that will eliminate the need for sophisticated equipment or trained personnel for conducting assays in resource-limited point-of-care locations (POC). Successful paper-based diagnostics have the potential to create a positive impact in both personal and global healthcare by increasing access to diagnostics, reducing cost and improving patient compliance. Several types of paper diagnostics have been developed, including paper chromatography, dipstick, and lateral-flow immunoassays. Additionally, many different analytical techniques, including chromatography ${ }^{1}$, immunoassays ${ }^{2,3}$, electrochemical probes $^{4,5}$, particulate metal testing ${ }^{6}$, and clinical chemistry assays ${ }^{7}$ have already been developed for paper-based devices. In some cases, paper-based tests provide qualitative results for routine organic and inorganic chemical analysis ${ }^{1}$. Other paperbased diagnostic tests have been combined with telemedicine-the use of existing communication infrastructure (eg. cell phones)—to perform quantitative tests and allow for experts to communicate results and treatment options to untrained users in the field. 
Microfluidic paper-based analytic devices (microPADs) are relatively novel, lowcost tools that combine features from microfluidic, lateral flow, and dipstick assays to conduct efficient paper-based POC diagnostic assays. Traditional microfluidic devices rely on devices made by etching channels into glass, silicon or poly(dimethylsiloxane) (PDMS) and typically require a pump to move fluids through the device. ${ }^{8}$ MicroPADs, on the other hand, are made by patterning hydrophilic porous membranes (e.g., paper) with hydrophobic barriers to create hydrophilic channels in which aqueous samples can wick via capillary action. Diagnostic assays are conducted on microPADs by wicking aqueous analytes into different patterned zones where they can react with other pre-deposited reagents. These devices effectively eliminate the need for an active pumping component generally associated with traditional microfluidic devices and are also much cheaper and easier to fabricate than conventional microfluidic devices.

Several methods of patterning microPADs exist, including photolithography and wax printing. ${ }^{9,10}$ Each method has its own trade-offs between time efficiency, cost efficiency and resolution. The concept of microPADs was founded upon the use of selective photolithographic techniques to create a hydrophobic pattern onto filter paper with photoresist. In comparison, microPADs prepared via wax printing require fewer steps for fabrication. Wax is simply printed on the surface of a piece of paper and then baked through the paper substrate. Although this method is simpler, wax printing suffers from lower resolution of the device as a result of lateral wax movement during the baking 
process. Figure 1 shows the two primary methods of microPAD preparation via photolithography or wax printing.

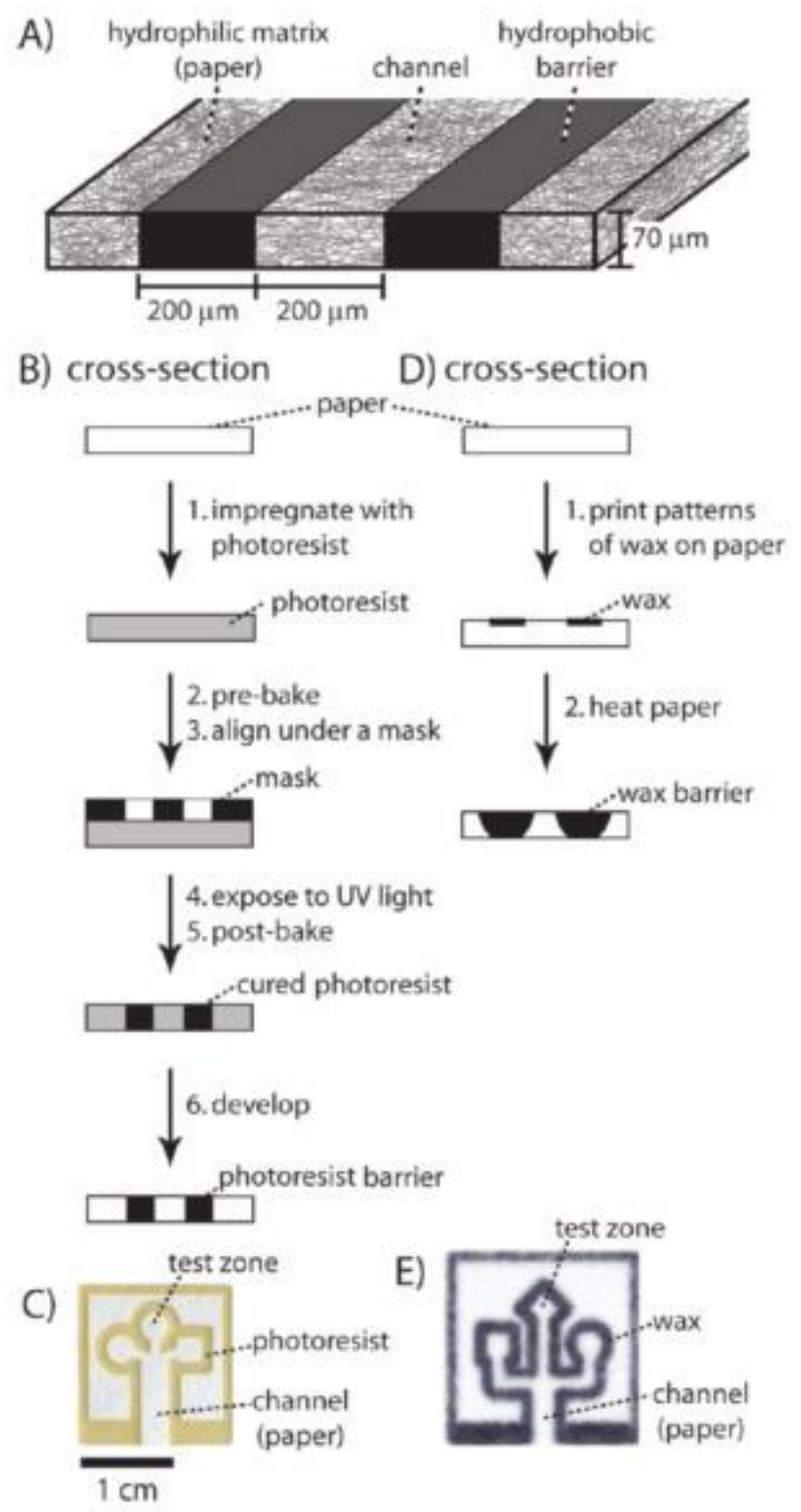

Figure 1. Fabrication of reagent pencils via photolithography or wax printing. ${ }^{11}$ 
The first microPADs were developed for detecting glucose and protein samples at relevant concentrations within the same range as commercially available assays. ${ }^{1}$ Since their conception, many other practical and quantitative uses of microPADs have been developed, including assays for blood typing ${ }^{12}$, urinalysis, and other bioanalyte detection. A recent surge of research focused on the development microPADs has established their versatility as a platform for conducting both qualitative and quantitative assays.

Some of the advantages of microPADs compared to other types of point-of-care diagnostic devices are that they can be quickly adapted to incorporate many different available technologies, the infrastructure required for their production is minimal (eg. printer for patterning) and prototyping microPADs from design to product is a fast process (less than a day). In this respect, successful applications of microPADs can be scaled up to large production quantities with both time and cost efficiency. Further, microPADs use small volumes of sample and have the potential to test detect multiple analytes simultaneously. As mentioned previously, patterns can be created with different established printing techniques including inkjet, photoresist, wax, and laser printing to create solid or permeable hydrophobic barriers. ${ }^{9}$ Additionally, paper itself is already available in a wide range of defined pore sizes and is engineered with different properties. While much of the work with microPADs uses filter paper or chromatography paper to fabricate the devices, it is also possible to fabricate microPADs using more sophisticated 
porous membranes such as nitrocellulose, or less sophisticated types of paper such as paper towels. ${ }^{11}$

Examples of three-dimensional (3D) microPADs have also been described, where sequential layers of patterned paper are stacked on top of each other to enable fluid flow in three dimensions. ${ }^{13}$ As a result, 3D microPADs have the capacity for higher throughput screening than 2D devices and can perform more sophisticated tests such as multi-step assays. The layers in a 3D device can either be permanently bonded together using permanent adhesives, or can be held together temporarily using a manifold or removable adhesives. The advantage of using a manifold or removable adhesives is that the layers of paper can be separated after an assay to obtain results from the middle layers of a device.$^{13}$ Figure 2 depicts an example of 3D microPAD where the layers were bonded together permanently using laser-printed toner as a thermal adhesive.

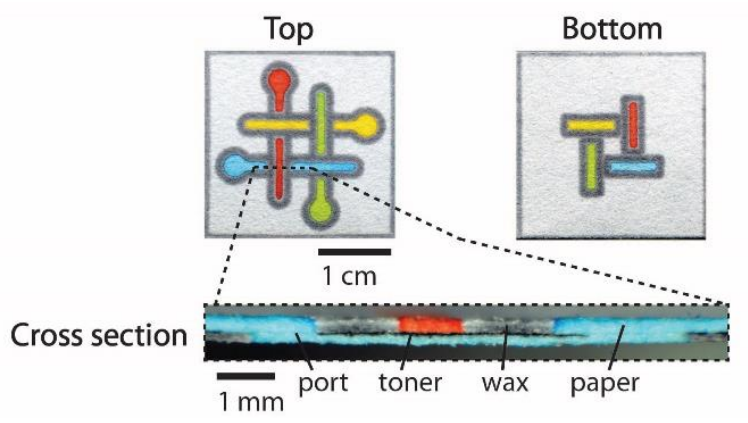

Figure 2. A three dimensional microPAD. ${ }^{13}$

It is important to note that the features of microPADs (eg. channels, deposition zones) are larger than classic microfluidic devices. Channels are often $\sim 1-2 \mathrm{~mm}$ in width 
and up to several centimeters in length, and sample volumes required for microPADs are typically in the range of 1-100 microliters. Therefore, in terms of the dimensions of the channels, microPADs are not microfluidic devices in the traditional sense. However, microPADs find relevance in the field of microfluidics for generally operating within the same laws of fluid physics as most microfluidic devices do. Surface chemistry effects that control fluid motion at small volumes are at play in both microfluidic devices and microPADs.

\subsection{Reagent Pencils}

Despite their simplicity, pencils are portable, low cost writing tools that have been developed for various applications beyond scripture. Typically, pencils are compressed mixtures of clay or polymeric binders and graphite. The ratio of graphite to clay or polymer determines the hardness of the pencil on a scale from $9 \mathrm{H}$ to $1 \mathrm{H}$ and $1 \mathrm{~B}$ to $9 \mathrm{~B}$, where $\mathrm{H}$ corresponds to a harder pencil, and B corresponds to softer pencils. Graphite incorporated into pencils can serve as electrical conductors when deposited onto a substrate. The use of pencils has led to the development of paper-based supercapacitors ${ }^{14}$, photoconductive UV sensors ${ }^{15}$, and electrokinetic-driven microfluidic systems ${ }^{16}$. The attractive qualities of pencil based applications for POC testing has led to a proliferation of research in the field. Based in their previous success, new applications of pencils have been developed for use with microPADs. 
In an effort to make microPADs even more accessible to POC locales, a novel method of storing sensitive reagents in pencils was developed. Although microPADs generally only require microgram quantities of reagents to perform an assay, the reagents must be stable for long periods of time at room temperature in order to be shipped to and used in remote settings. Specifically, enzymes involved in biochemical assays are prone to denature in suboptimal conditions, effectively limiting the potential of microPADs to be deployed to remote locations. As a way of improving the shelf life of reagents for use with microPADs, reagent pencils offer a solvent-free, simple method of storing and depositing sensitive reagents onto mciroPADs. ${ }^{11}$ To make reagent pencils, a particular reagent or set of reagents is combined at up to $15 \% \mathrm{w} / \mathrm{w}$ with a mixture of $75: 25$ polymer:graphite and compressed into a cylindrical pellet. Reagent pencils loaded into mechanical pencil holders then allow reagents to be deposited in a solid state onto microPADs.

Reagent pencils offer advantages in their rapid fabrication, solvent-free deposition, low production cost, and reagent stabilization. Previous work has shown that horseradish peroxidase (HRP) was able to undergo the fabrication process with minimal effect on its activity. Further, HRP retained activity even after 42 days stored in a pencil core. In comparison, HRP deposited on microPADs via solution deposition showed a decline in signal intensity after 3 days stored on paper. ${ }^{11}$ Similar results were found for tests conducted on alkaline phosphatase (ALP). The results of these experiments are shown in Figure 3. 

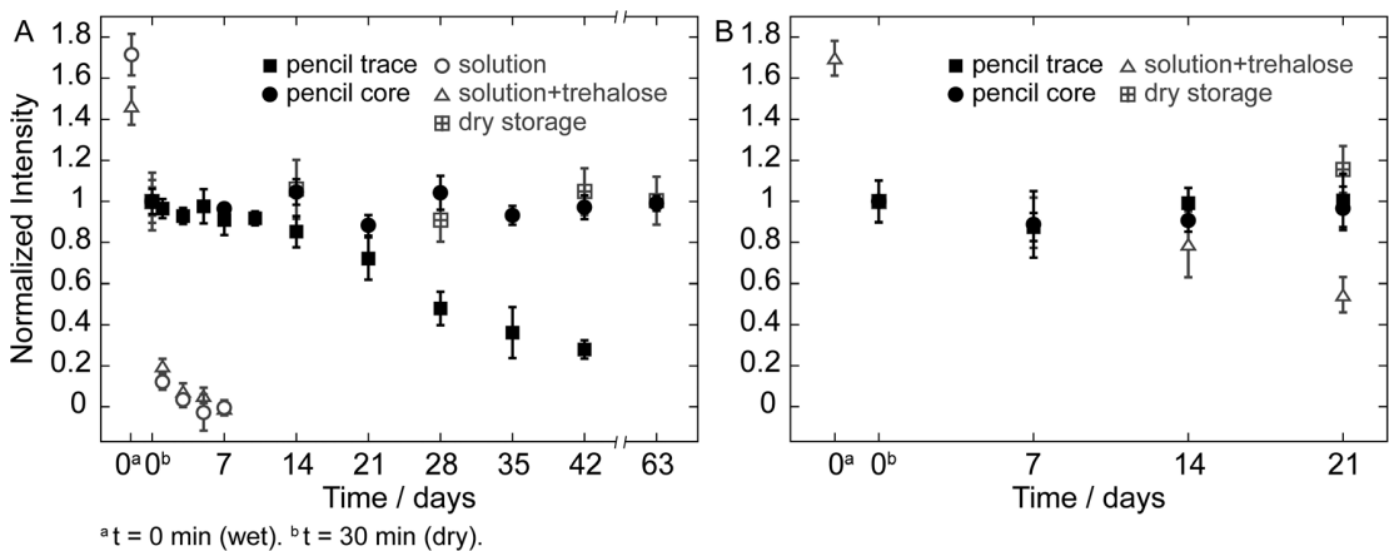

Figure 3. Shelf life of (A) HRP and (B) ALP in different formats, including pencil trace, pencil core, solution, and dry storage. ${ }^{11}$

The implications of these preliminary results suggest that reagent pencils show promise for overcoming challenges of reagent shelf life and stability. Their use in conjunction with microPADs increases their attractiveness for POC technology. Because reagent pencils are relatively novel in concept, further work must be done to refine the pencil system. Possible routes of investigation include improving the release efficiency of reagents, modifying the polymer matrix used, and characterization other small molecules and enzyme behavior in reagent pencils.

Because this work extensively uses HRP and ALP as a model enzyme loaded into reagent pencils, it is important to discuss the characteristics, function and applications of HRP and ALP before discussing their role in microPADs. 


\subsection{Horseradish Peroxidase (HRP) Structure, Function and Applications}

Horseradish peroxidase has been studied extensively in the realm of diagnostics and biochemistry for many decades and serves as the primary model enzyme for peroxidases. Peroxidases are found in a plethora of living organisms and catalyze the electron transfer from substrate molecules to peroxide species $\left(e g . \mathrm{H}_{2} \mathrm{O}_{2}\right)$. HRP in particular belongs to the ferroprotoporphyrin group of peroxidases. Structurally, HRP is a single chain polypeptide that contains four disulfide bridges. The majority of studies on HRP stem from the C1A isoenzyme, and its structure is shown below in Figure $4 .{ }^{17} \mathrm{HRP}$ is a globular molecule whose proximal and distal regions are separated by a coordinated heme group. Typically, the relative purity of HRP is evaluated based on the ratio of $A_{403}$ to $A_{280}$, or the absorbances of heme and of the aromatic amino acids present in HRP. Further, HRP C1A is known to have three surface lysine residues available for chemical modification. ${ }^{18}$ This becomes important in studies involving enzyme immobilization via covalent linkages.

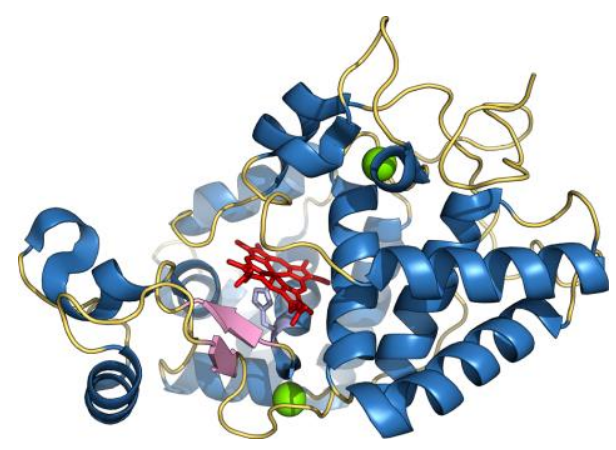

Figure 4. HRP C1A isoenzyme structure. 
HRP has been used extensively as a reporter enzyme in diagnostic assays, in the biocatalysis of polymerizations, and in bioremediation systems for the removal of contaminants. It is also utilized widely in immunology as a label for immunoglobulins as a result of its small size and stability as compared to other enzyme labels. For this work, 2,2'-azino-bis(3-ethylbenzothiazoline-6-sulphonic acid) (ABTS) is used as a water soluble HRP substrate that yields a radical product that appears green and has two major absorbance peaks at $410 \mathrm{~nm}$ and $650 \mathrm{~nm}$. On a microPAD, this color signal can be developed rapidly over the course of 30 minutes and scanned for color signal intensity after the device has dried. Figure $\mathbf{5}$ depicts the conversion of ABTS to its green radical product by HRP.<smiles>CCn1/c(=N/N=c2\sc3cc(S(=O)(=O)O)ccc3n2CC)sc2cc(S(=O)(=O)O)ccc21</smiles>

Figure 5. Conversion of ABTS in the presence of $\mathrm{H}_{2} \mathrm{O}_{2}$ by HRP yields a radical product that appears green and absorbs at $410 \mathrm{~nm}$ and $650 \mathrm{~nm}$.

It is also important to note that HRP can be stabilized and protected from reactive oxidative species by incorporating poly(ethylene glycol) (PEG). ${ }^{19}$ As previously discussed, 
reagent pencils were shown to extend the shelf life of both HRP and ALP. It is possible that both enzymes were protected from reactive oxidative species because of the incorporation of PEG into reagent pencils, thereby extending the shelf life of each enzyme. This is an important consideration when designing microPADs with HRP in mind.

\subsection{Experimental Plan and Overview}

The optimization and characterization of reagent pencils from its original design has great potential to improve the performance of microPADs. The original reagent pencils were made from a mixture of PEG 2000 -graphite at a $25: 75$ ratio with up to $15 \%$ $\mathrm{w} / \mathrm{w}$ of reagent added. Although this worked well for the purposes of introducing the concept and methodology of reagent pencils, little work investigated their specific characterization and efficacy. Understanding the release mechanism of reagents from the polymer matrix onto chromatography paper will help determine efficient design of reagent pencils. Further, this will allow for the development of more precise, accurate, and sophisticated devices.

In order to achieve this goal, this work aimed to understand the wear, release characteristics, and release efficiencies of reagent pencil systems. Variable components were tested, including pencil diameter, polymer matrices, reagent types, reagent content, and graphite content. Effects of polymer components on reagent pencil performance were investigated via varying the molecular weights of poly(ethylene glycol) (PEG), varying 
polymer chain end groups, and investigating composite systems of PEG and poly $(\mathrm{N}$ isopropylacrylamide) (PNIPAM). In particular, reagent type was also of significant importance. Thus, the release behavior of model small molecules, polymers, and enzymes deposited via reagent pencils was investigated. Reagents studied included an erioglaucine disodium salt (a blue dye, BD), glucose, HRP, ALP, and PNIPAM. By extensively investigating the behavior of the polymer matrix and reagent type in the reagent pencil, characteristics of ideal reagent pencils may be better understood.

In a similar vein, the effects of PEG on HRP and ALP stabilization were investigated in order to fully understand previous work that has shown the extension of HRP shelf life when stored in a reagent pencil. In particular, the effects of solid and solution deposition of PEG, molecular weight, and concentration are investigated across different concentrations of HRP.

The ultimate goal of all this work is to enable the development of akit of microPADs and reagent pencils that can be taken to a low-resource POC setting and used to conduct a variety of rapid and quantitative diagnostic assays. With the introduction of reagent grafting tools, devices can be customized on site as needed to meet the demands encountered at the point of care. 
CHAPTER 2. CHARACTERIZATION OF POLYMER COMPOSITION, REAGENT TYPE, AND RELEASE EFFICIENTY FOR REAGENT PENCILS

\subsection{Characterization of reagent pencils}

2.1.1. Characterization of PEG and PEG derivative reagent pencil systems

Many polymers, including poly(methyl methacrylate) (PMMA), poly(vinyl alcohol) (PVA), and polystyrene (PS), and PEG were viable candidates as the polymer component in reagent pencils. Initially, PMMA, PVA and PS were considered as potential candidates for reagent pencils, but these polymers created fragile pencil cores that crumbled easily and could not withstand the pressing process of fabricating a reagent pencil. PEG was chosen as the primary polymer component for reagent pencils largely due to its wide range of molecular weights available, low cost, and its ability to withstand pellet pressing and rotary evaporation at a high temperature. Further, while PEG is hygroscopic at low molecular weights, it is not hygroscopic at molecular weights $\geq 2000 \mathrm{~g} / \mathrm{mol}$ so it could be stored under ambient conditions.

This work focused on the use of PEGs with molecular weights $\left(M_{n}\right)$ between 2000 and $6000 \mathrm{~g} / \mathrm{mol}$. In general, reagent pencils made the use of low molecular weight PEG resulted in softer reagent pencil cores, while the use of high molecular weight PEG resulted in harder, but also more brittle and fragile, pencil cores. This trend is agreeable with the Flory-Fox equation which relates $M_{n}$ to glass transition temperature $\left(T_{g}\right)$ and free 
volume. $^{8-9}$ PEG-graphite systems that incorporate greater $M_{n}$ PEG have limited segmental mobility in polymer chains, giving it a more brittle character at room temperature. This trend is also agreeable with the melting temperatures $\left(T_{m}\right)$ of PEG obtained from differential scanning calorimetry (DSC) measurements, summarized in Table 1. As $M_{n}$ of PEG increased, $T_{m}$ also increased. This implies that higher $M_{n}$ PEG chains require more energy to undergo a phase transition and break intermolecular forces between polymer chains that are packed tightly together.

Table 1. Tm of PEG-graphite systems via DSC.

\begin{tabular}{cc}
\hline$M_{n}$ PEG (g/mol) & $\mathrm{T}_{\mathrm{m}}\left({ }^{\circ} \mathbf{C}\right)$ \\
\hline 1000 & 34.94 \\
1500 & 46.27 \\
3400 & 53.05 \\
6000 & 61.30 \\
\hline
\end{tabular}

Reagent pencil cores were fabricated in two different diameters of 6.35 and 3.00 $\mathrm{mm}$, respectively. Figure 6 shows the deposition of the same PEG-graphite mixture using the two different diameter cores, and the deposition characteristics appear to be different when viewed under a microscope. The 6.35-mm PEG 2000-graphite core (Figure 6A) deposited less material onto the paper than the 3-mm core (Figure 6B) or a 3-mm PEG 6000-graphite core (Figure 6C) pencil cores. 

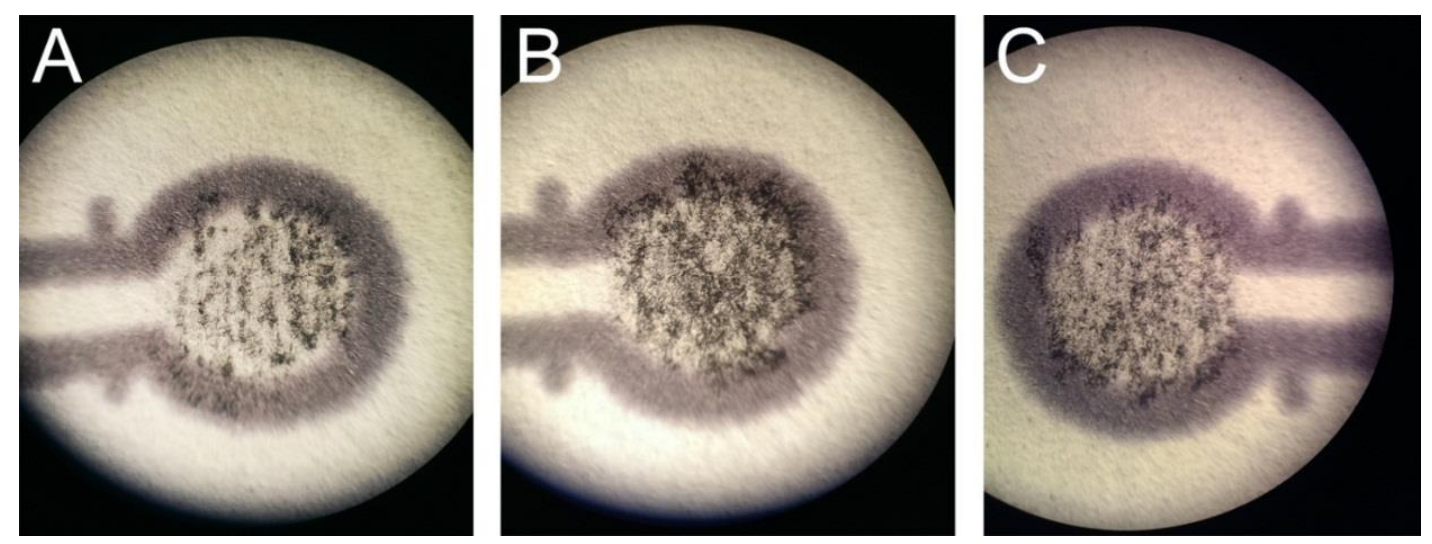

Figure 6. Comparison of PEG-graphite pencil deposition at different diameters under a microscope where (A) PEG 2000, $6.35 \mathrm{~mm}$ visibly deposits less reagent than (B) PEG 2000, $3.00 \mathrm{~mm}$ and (C) PEG 6000, $3.00 \mathrm{~mm}$ reagent pencils.

In order to quantify this observation, the wear resistance of various PEG-graphite systems was measured at a constant velocity, force and distance using a retrofitted cutting plotter. Figure 7 illustrates the results of this experiment. Overall, the 3-mm cores showed greater wear than the $6.35-\mathrm{mm}$ cores. This result demonstrates that pencil cores with smaller diameters are able to deposit a greater amount of reagent onto microPADs as compared to pencil cores with larger diameters. Additionally, regardless of core diameter, the wear of the pencils decreased as $M_{n}$ of the PEG used to make the core increased. The wear of cores that were fabricated using a combination of two different $\mathrm{Mn}$ PEG components was approximately equal to a weighted average of the wear determined for the individual PEG components. The hardness of the PEG-graphite system, then, can be customized by either varying the $M_{n}$ of PEG or changing the diameter of the pencil core itself. The 10\% PEG 600/ 90\% PEG 6000 system displayed the greatest wear 
amongst all other pencil core systems. The incorporation of PEG 600 plasticized the pencil core significantly, however, and contributed to a large margin of error as a result of its softer characteristic. For simplicity, pencil cores utilizing only PEG 2000 were determined to have the best performance for its greater wear and consistency.

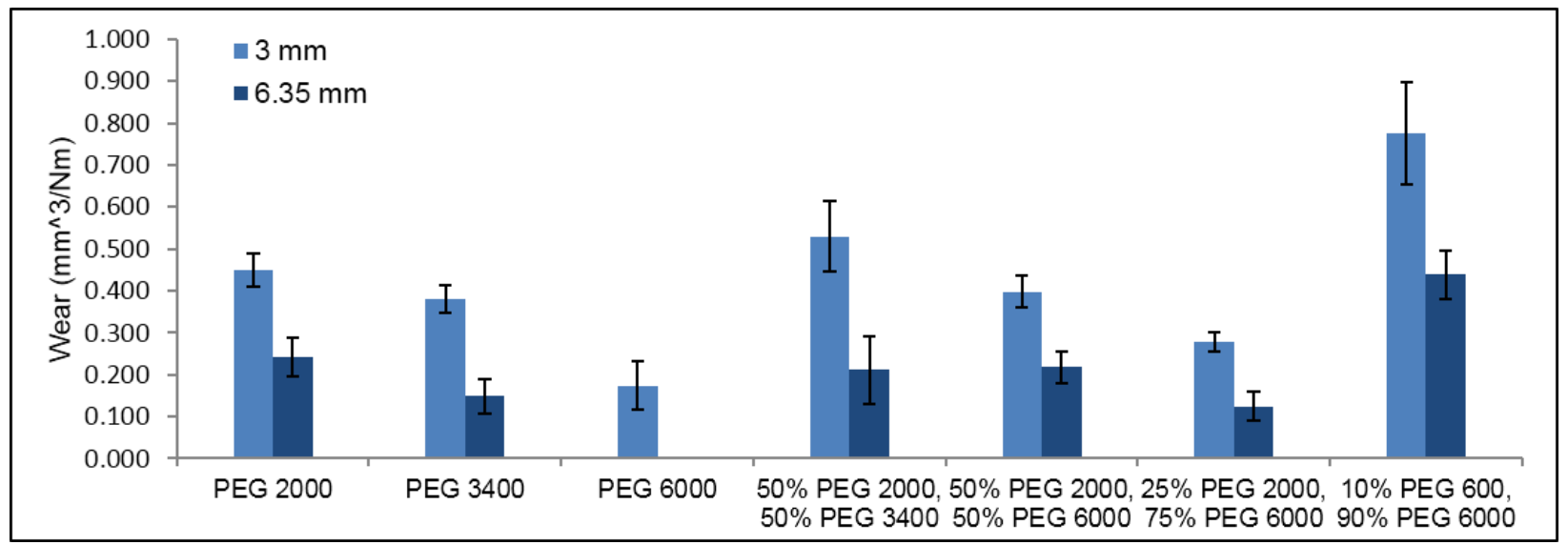

Figure 7. Wear comparison of PEG-graphite pellets at varying compositions and pellet diameters. All pencil formulations did not incorporate blue dye. $6.35 \mathrm{~mm}$ PEG 6000 was too brittle to be pressed into a pencil core.

2.1.2. Characterization of erioglaucine disodium salt reagent pencils

In order to understand the effect of reagent incorporation on wear, functionalized PEG derivatives, including PEGME and PEGdiME $\left(M_{n}=2000\right)$, were tested for wear with and without the incorporation of $15 \% \mathrm{w} / \mathrm{w}$ erioglaucine disodium salt as shown in Figure 8. The pencil core with PEG and PEGME showed a significant difference in wear with the inclusion of blue dye while the PEGdiME pencil core showed no significant change in wear with the inclusion of blue dye. This suggests that the functionalization of PEGdiME gives it a more inherent lower wear with no reagent present. The presence of BD also 
does not change the wear. This suggests that the functionalization of the polymer can dictate the wear of the reagent pencil, regardless of the reagent itself. Interestingly, this effect is not observed for PEGME systems. It is possible that simply more methyl ether chain ends need to be present, as in PEGdiME systems, before this effect is observed.

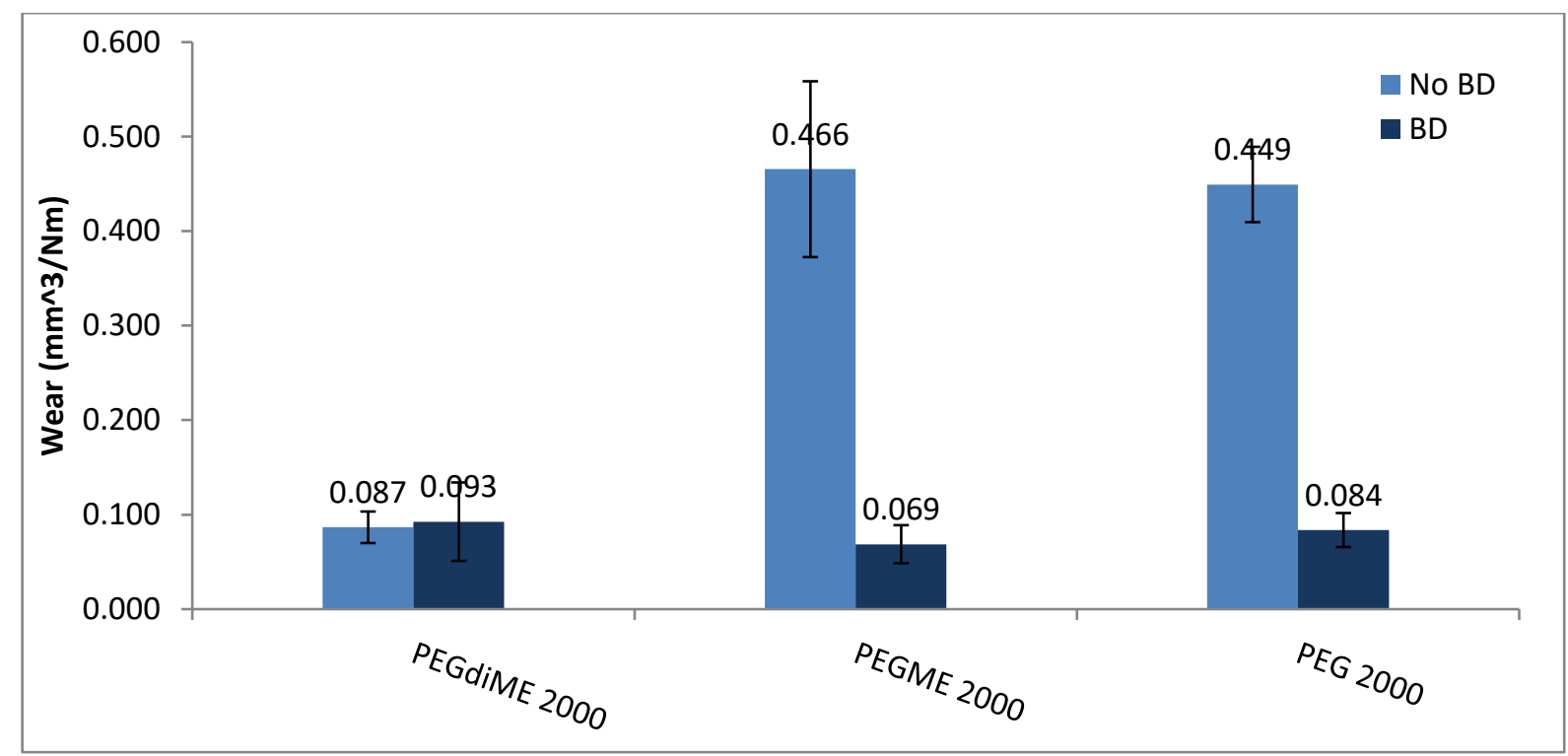

Figure 8. Investigation of PEG functionalization as PEGME and PEGdiME on wear both with and without $15 \% \mathrm{w} / \mathrm{w}$ blue dye.

The effect of blue dye incorporation on wear was also tested with varying concentrations of the reagent on different molecular weight PEG pencils, as shown in Figure 9. PEG 2000, PEGdiME 2000, and PEG 6000 systems at 1, 5, 7.5, 10, and 15\% w/w blue dye were tested for their wear. Pencil cores with PEG 2000 and PEGdiME 2000 saw a significant decrease in wear as blue dye concentration increased. This effect is attributed to the tackiness of the reagent pencil at higher concentrations of blue dye. 
Because erioglaucine disodium salt is a bulky molecule, it is not as easily distributed amongst a polymer matrix at higher concentrations of its incorporation. This gives the reagent pencil a clay-like feel when delivered to microPAD devices, and consequently leads to less efficient delivery of reagent onto paper. Interestingly, there is no noticeable change in wear with changing blue dye concentration for PEG 6000 systems. This indicates that the bulky nature of the blue dye does not noticeably affect the wear of PEG 6000 pencil core systems. It seems that longer PEG chains are able to more easily disperse the blue dye than shorter PEG chains, giving the pencil core a more brittle characteristic. Although this quality may be desirable, the brittle, scratchy nature of the PEG 6000 pencil core on microPADs restricts its utility as a reagent pencil. More importantly, however, this test suggests that the size of the reagent can also dictate the properties of the reagent pencil as significantly as the length of the polymer can. While the storage of reagents in solid state may be convenient, there may be restrictions on the total volume of reagent that can be incorporated into reagent pencils if the average polymer chain length is too short. In other words, at some higher molecular weight of PEG the wear is dictated by the polymer chain length and not by the reagent itself. This is similar to what we have previously discussed, where there is some chain end functionality of the polymer that, at high enough frequency (as in PEG vs PEGME vs PEGdiME), the measurement of wear becomes dictated by polymer functionality as opposed to because of the properties of the reagent. 


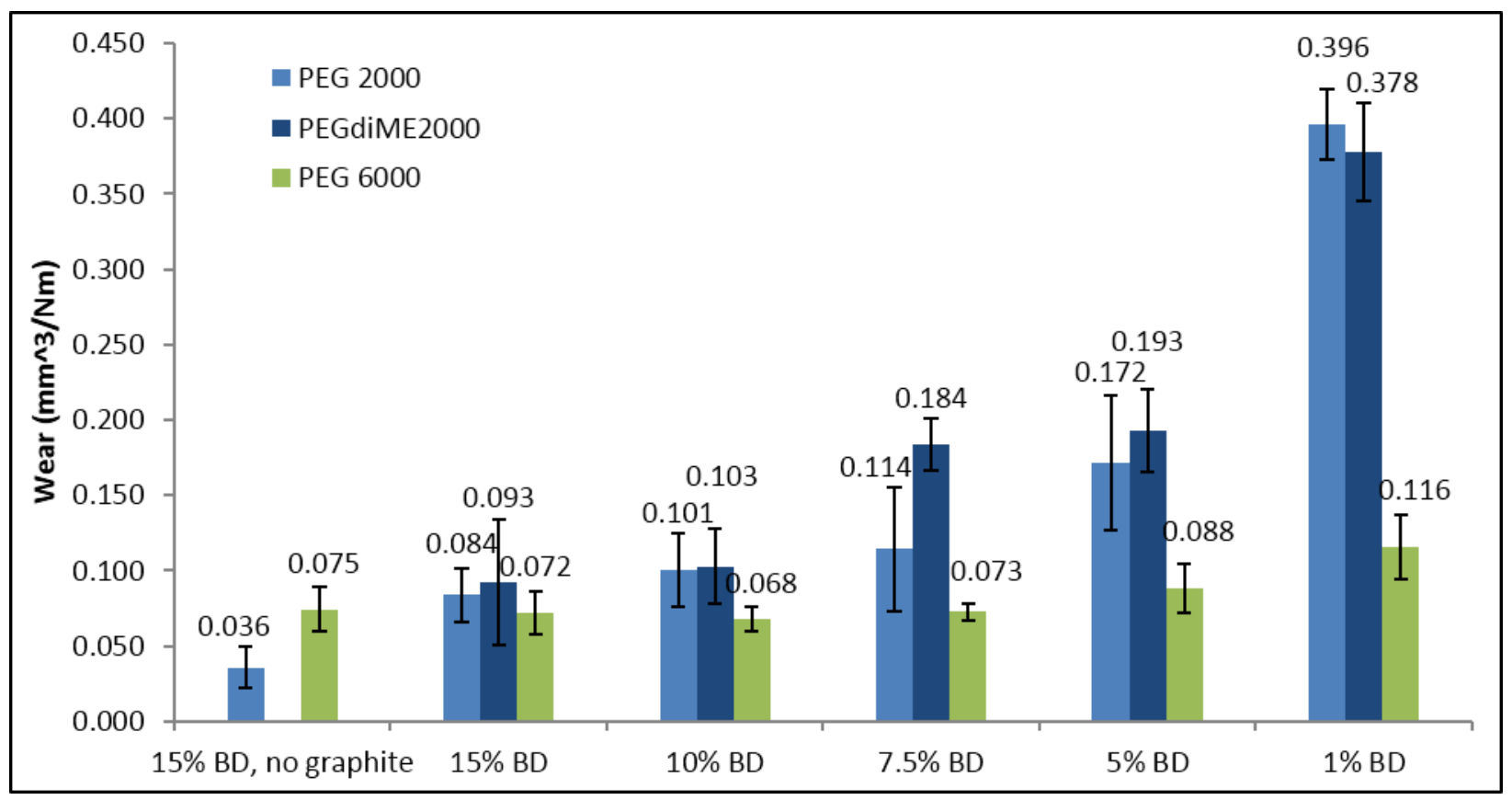

Figure 9. Wear measurements for PEG 2000, PEGdiME 2000, and PEG 6000 pencil cores with varying blue dye concentrations.

2.1.3. Colorimetric release profile for blue dye reagent pencil systems

To determine the effect of each system's composition on the release of reagent onto microPADs, water soluble erioglaucine disodium salt (a blue dye, BD) was incorporated into PEG-graphite systems at $15 \% \mathrm{w} / \mathrm{w}$ and a colorimetric release profile was conducted on 'bone' microPAD devices (Figure 23). Reagent pencil cores were loaded into mechanical pencil holders and delivered by hand into the sample addition zone. Then, $13 \mu \mathrm{L}$ of deionized water was pipetted over the sample addition zone and allowed to wick through the device and dry before subsequent scanning and analysis of the end zone in imaging software. Figure $\mathbf{1 0}$ depicts the results of the release profile test. All pencil cores showed statistically equivalent signal intensities despite different polymer 
matrix compositions. This suggests that the signal intensity was saturated at $15 \% \mathrm{w} / \mathrm{w}$ blue dye, and a different concentration of reagent is required to determine differences in reagent delivery performance based on pencil core composition. In order to determine the optimal operating concentration of blue dye, pencil cores were formulated with PEG 2000, PEGdiME 2000, and PEG 6000 at 1, 5, 7.5, 10, and 15\% w/w blue dye. Figure 11 shows the colorimetric release profile of these pencil core systems. Across all three polymer matrixes tested, signal saturation was apparent above $7.5 \% \mathrm{w} / \mathrm{w}$ blue dye incorporation. Above this concentration, the measured signal intensity was statistically equivalent regardless of polymer composition. For this reasoning, $5 \% \mathrm{w} / \mathrm{w}$ blue dye concentration in reagent pencils was determined to be the optimal operating concentration for understanding the effects of polymer matrix composition in relation to signal intensity in release profile tests. 


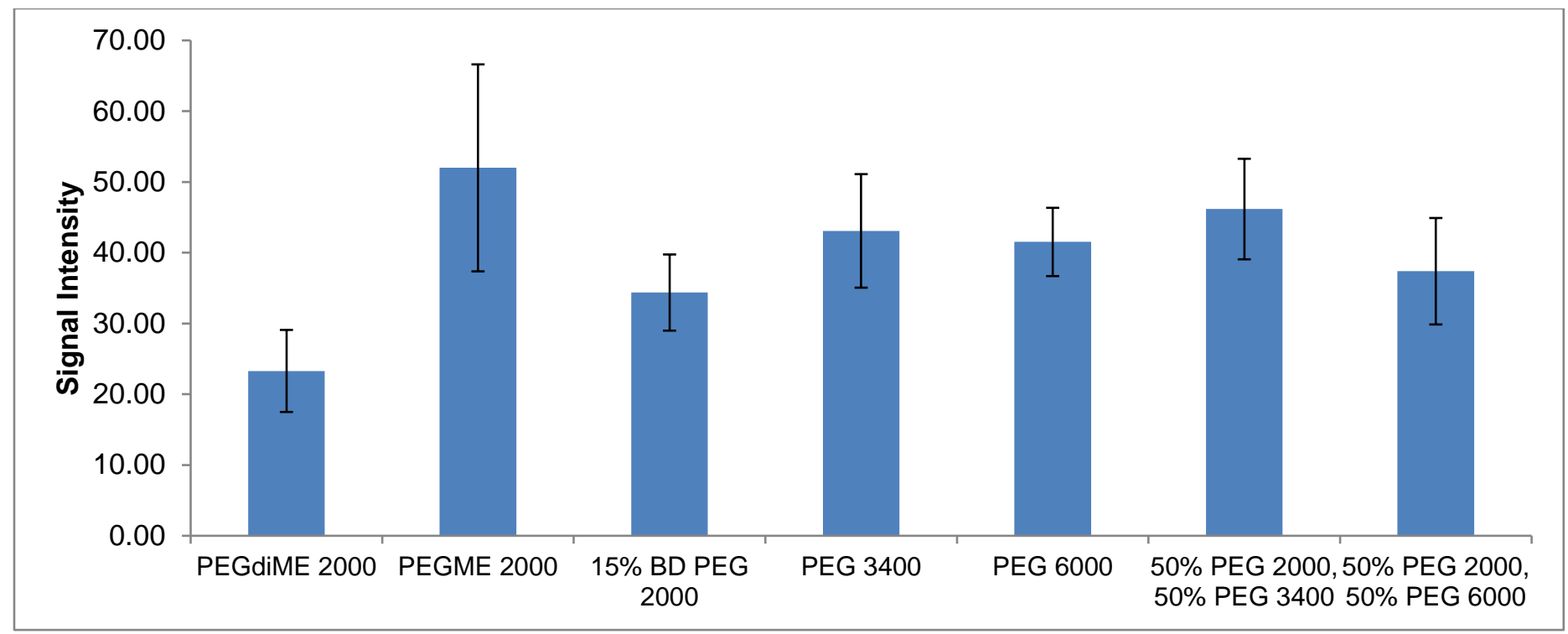

Figure 10. Comparison of $3 \mathrm{~mm} 15 \%$ blue dye PEG-graphite pencil core formulations via a colorimetric release profile test on microPADs.

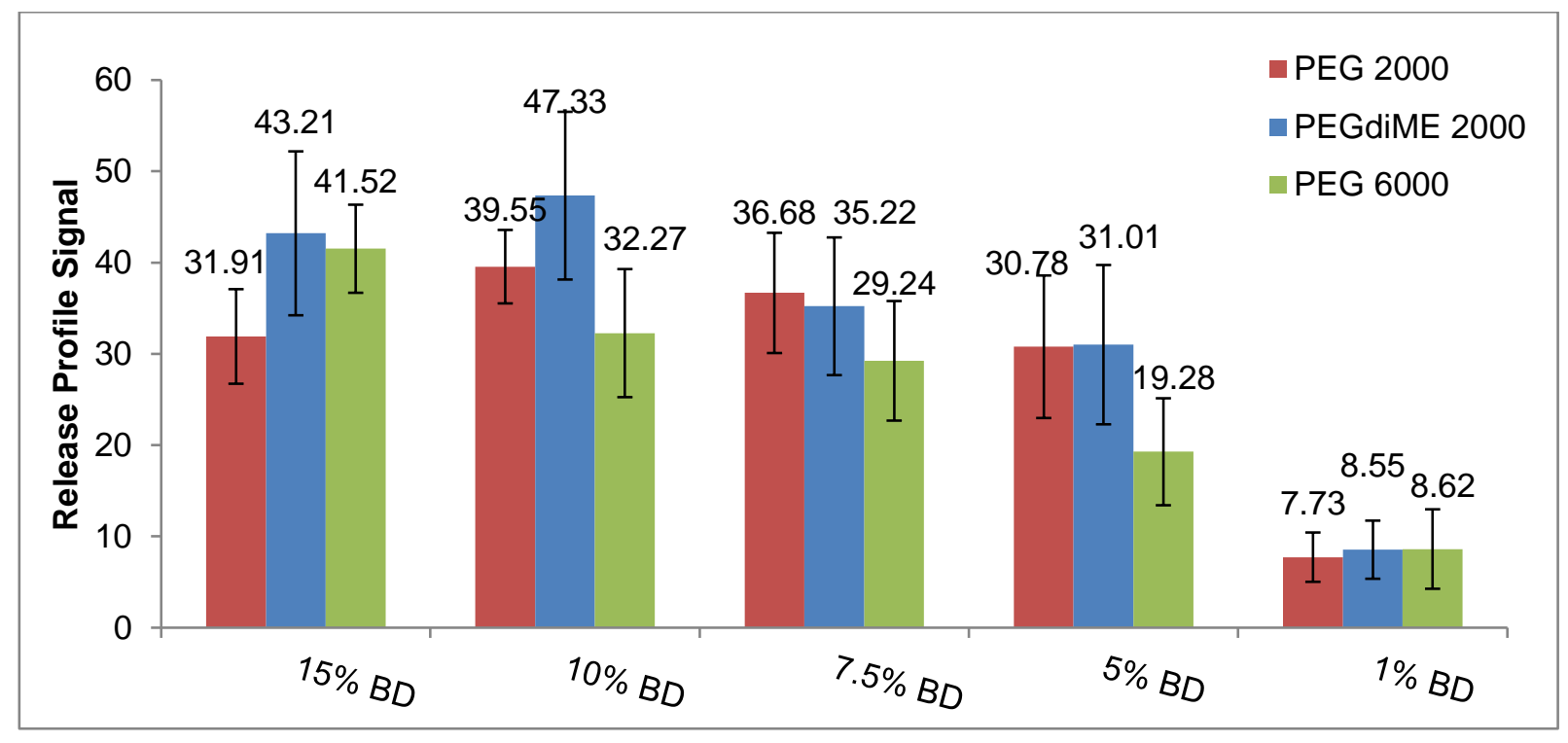

Figure 11. Release profile signal intensities for PEG 2000, PEGdiME 2000, and PEG 6000 pencil cores with varying blue dye concentrations. 
2.1.4. Characterization of blue dye, HRP, and glucose PEGdiME reagent pencils based on fabrication methodology

In order to be an effective reagent pencil, the fabrication process must not impact the function of the reagent. In the case of enzymes, they will most likely denature if put through the rotary evaporation fabrication method. It was necessary to compare the properties of reagent pencils fabricated via rotary evaporation versus the reagent pencils fabricated via lyophilization. Figure 12 compares the wear of $5 \% \mathrm{w} / \mathrm{w}$ blue dye/glucose/HRP PEGdiME reagent pencils fabricated by both rotary evaporation and lyophilization. For the blue dye systems, there was no significant change in wear regardless of fabrication method. Even though the lyophilization process typically leaves residual water in the reagent pencil, the wear for both systems was likely showed no difference as a result of the hygroscopic properties of the blue dye. For the glucose systems, however, a significant difference between methods is observed because of the water retention in the lyophilized pencil, and weaker hygroscopic properties of glucose. For HRP, the rotary evaporated sample was not taken because HRP is known to denature in acetone. 


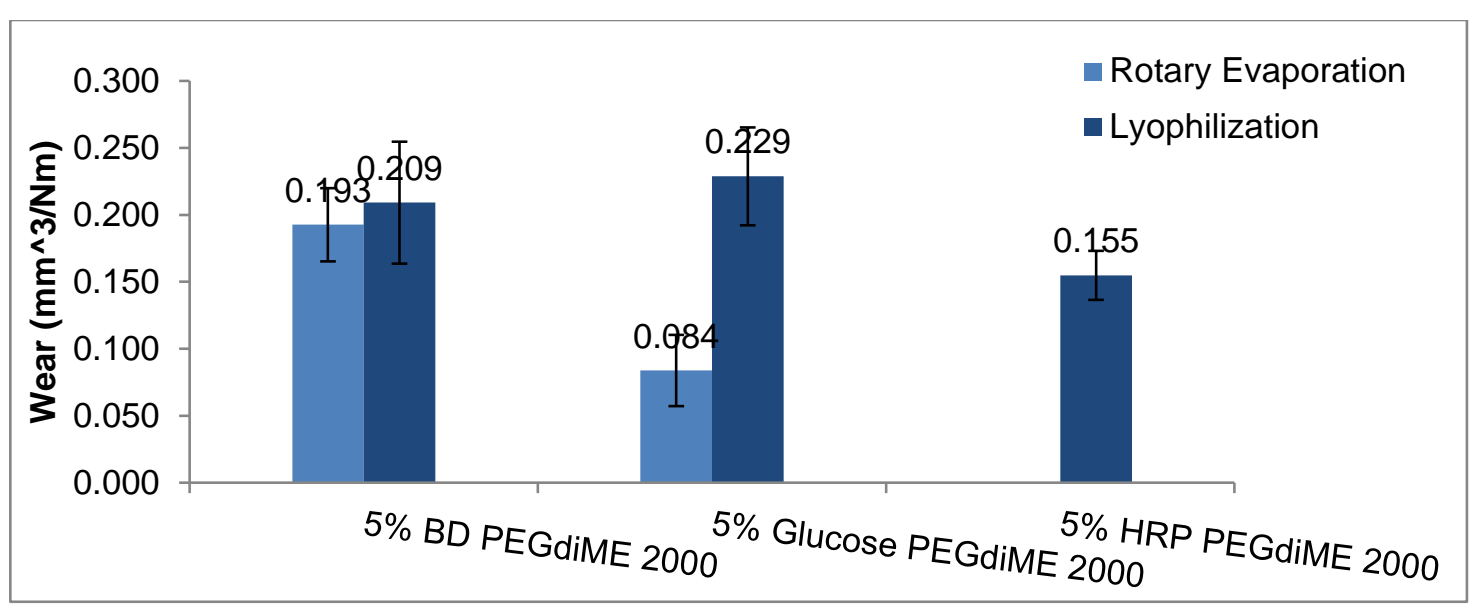

Figure 12. Comparison of wear for PEGdiME rotary evaporated and lyophilized pencil cores with $5 \% \mathrm{w} / \mathrm{w}$ of blue dye, glucose, or horseradish peroxidase.

2.2. Release efficiency of reagent pencils

In order to determine the efficiency of the reagent pencils, it was necessary to develop calibration curves for the colorimetric response of different reagents at known concentrations via solution deposition. Each test was run on a lateral flow bone device. The fitted curves for blue dye, HRP, and glucose solutions are shown in Figure 13, Figure 14, Figure 15, respectively. For the blue dye and HRP solution curves, a linear relationship between concentration and signal intensity was achieved by plotting the inverse colorimetric signal intensity against the inverse concentration of each respective reagent. The chosen range for glucose concentrations saw a direct linear relationship between signal intensity and reagent concentration and did not need to be replotted. 


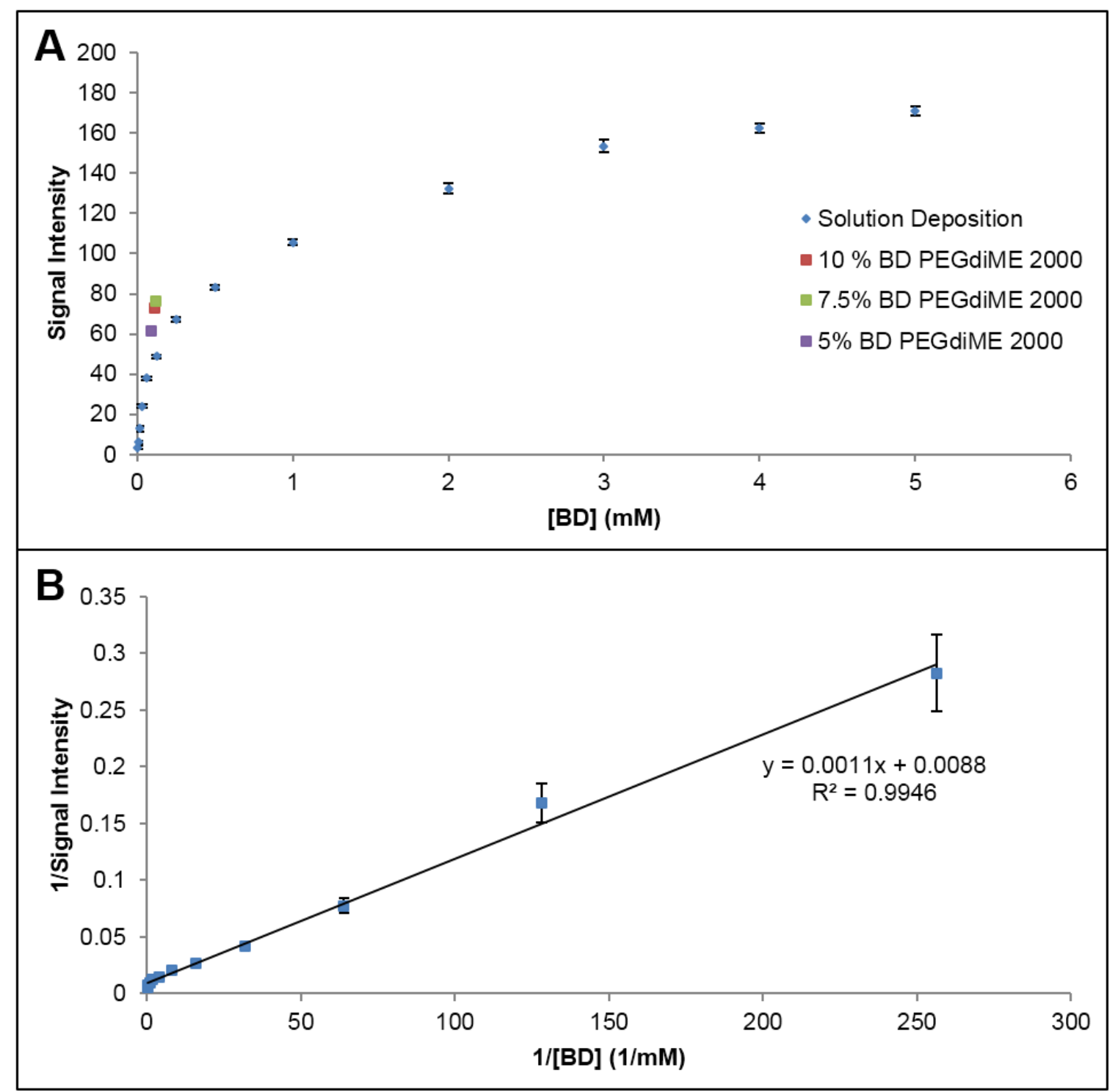

Figure 13. A) Colorimetric release profile and its B) reciprocal release profile for blue dye via solution deposition at known concentrations. Signal intensity for respective reagent pencils containing $10,7.5$, or $5 \%$ blue dye are compared release profile curve. 


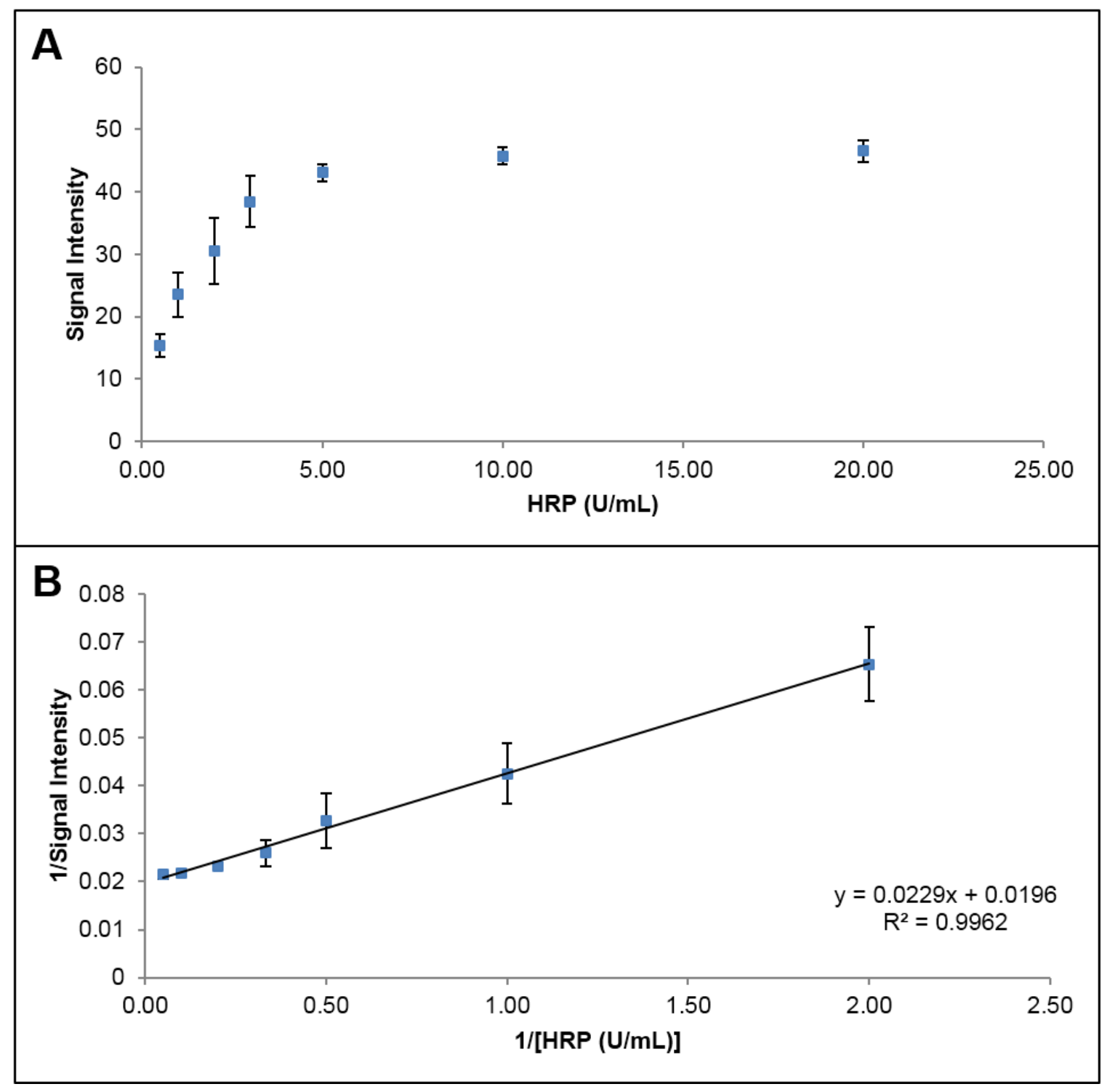

Figure 14. A) Colorimetric release profile and its $B$ ) reciprocal release profile for horseradish peroxidase via solution deposition at known concentrations on a 96 well microPAD. 


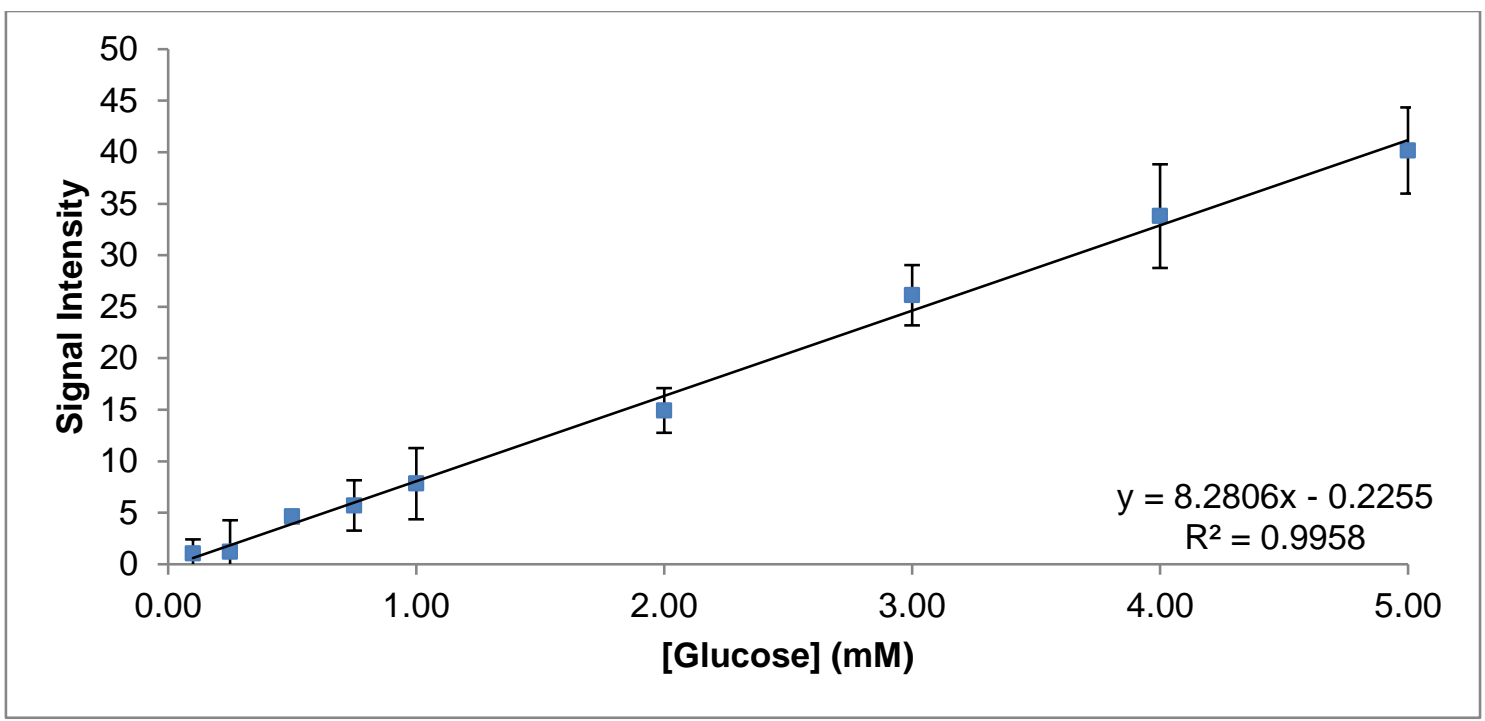

Figure 15. Colorimetric release profile for glucose via solution deposition at known concentrations.

From the curves developed via solution deposition, it is possible to derive the release efficiency of various reagent pencils. The theoretical concentration of reagent delivered onto lateral flow microPADs via reagent pencils could be derived by measuring the change in mass before and after deposition. This value can be compared to the experimental reagent concentration extrapolated from the signal intensity. Thus, the efficiency of different reagent pencil systems can be determined. The release efficiencies of blue dye, HRP, and glucose reagent pencils at varying concentrations are shown in Figure 16. Overall, regardless of reagent concentration, the efficiencies of glucose reagent pencils remain relatively consistent at $\sim 30-40$ percent. The colorimetric signal intensities corresponding glucose reagent pencils are shown in Figure 17. For the 5\% $\mathrm{w} / \mathrm{w}$ sample, there is a significant drop in signal intensity for both PEG and PEGdiME 
glucose pencils. This result was unexpected, and will need to be redone in the future to reassess its validity. Overall, however, the performance of glucose pencils and their release profiles indicates that regardless of the colorimetric response of the reagent pencil, the overall efficiency of the pencil itself is maintained within the same system. Additionally, utilizing PEG or PEGdiME has no significant effects on the overall release efficiency of glucose.

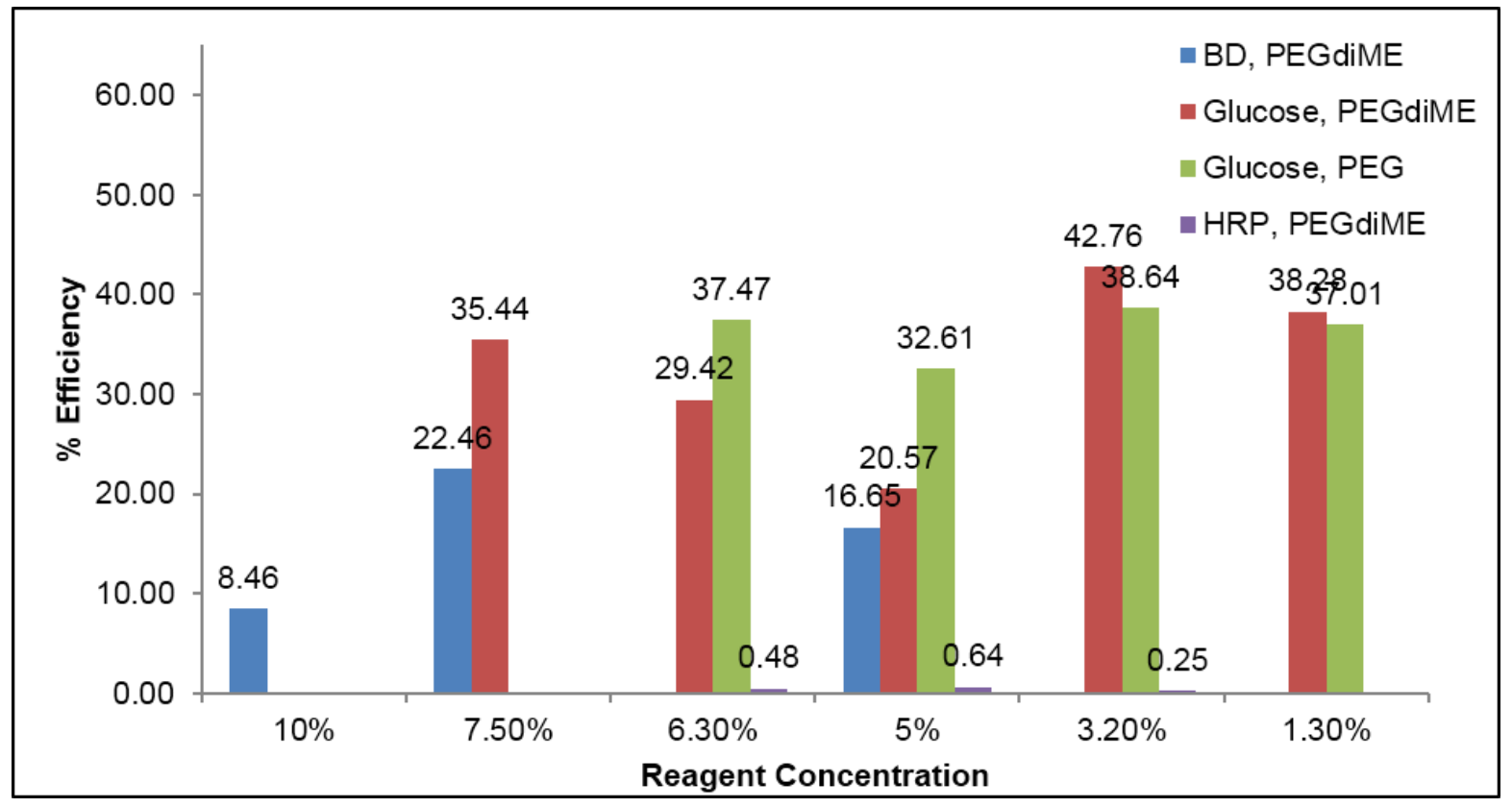

Figure 16. Efficiency of blue dye and glucose reagent pencils with PEGdiME 2000 as polymer component of pencil core. 


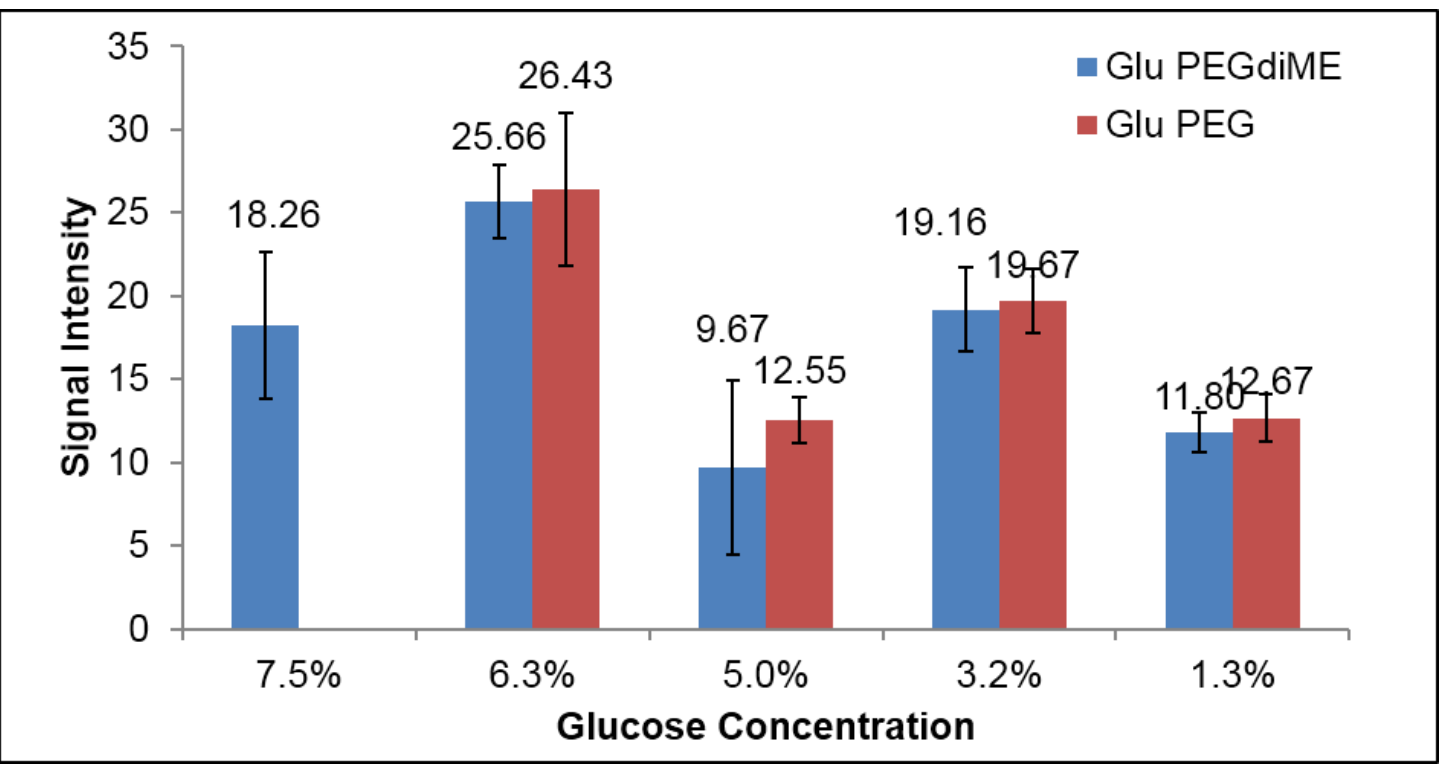

Figure 17. Signal intensity of PEG and PEGdiME reagent pencils with varying glucose concentration.

\subsection{Solution and Solid Polymer Stabilization of HRP and ALP}

While previous work has indicated that reagent pencils stabilize enzymes in the solid state, few literature discusses the extent of the stabilization characteristics that result from the incorporation of PEG. Herein, we discuss the role of PEG molecular weight and its effects on HRP and ALP activity.

\subsubsection{HRP Stabilization}

In order to understand the effects of PEG in the reagent pencil on the activity of HRP, it is necessary to deconstruct the reagent pencil and only work with PEG without the presence of graphite. It was decided that the most efficient experimental method would utilize 96 well microPADs. The effects of PEG 400, 2000, 3400 were determined 
initially. In general, when no PEG is present, HRP activity at concentrations below $5 \mathrm{U} / \mathrm{mL}$ drops off. Figure 18 shows that the signal intensity of HRP at lower concentrations are significantly improved with the presence of PEG. Interestingly, this effect is observed with both solid and solution based deposition of PEG prior to the HRP assay. Note that in this case solid deposition of PEG is purely the polymer, and does not include any graphite. Further, this effect is also observed for a lower molecular weight PEG (PEG 400), which is liquid at room temperature. From this preliminary testing, PEG has an apparent positive effect on reinforcing the stability of HRP activity. Possibly, this supports the shelf life studies conducted on HRP reagent pencils. In general, the signal intensity of HRP becomes saturated above $5 \mathrm{U} / \mathrm{mL}$. Because the stabilization effect of HRP was observed at concentrations below $5 \mathrm{U} / \mathrm{mL}$, subsequent experiments evaluated HRP activity between 0.1 and $3 \mathrm{U} / \mathrm{mL}$ HRP. 


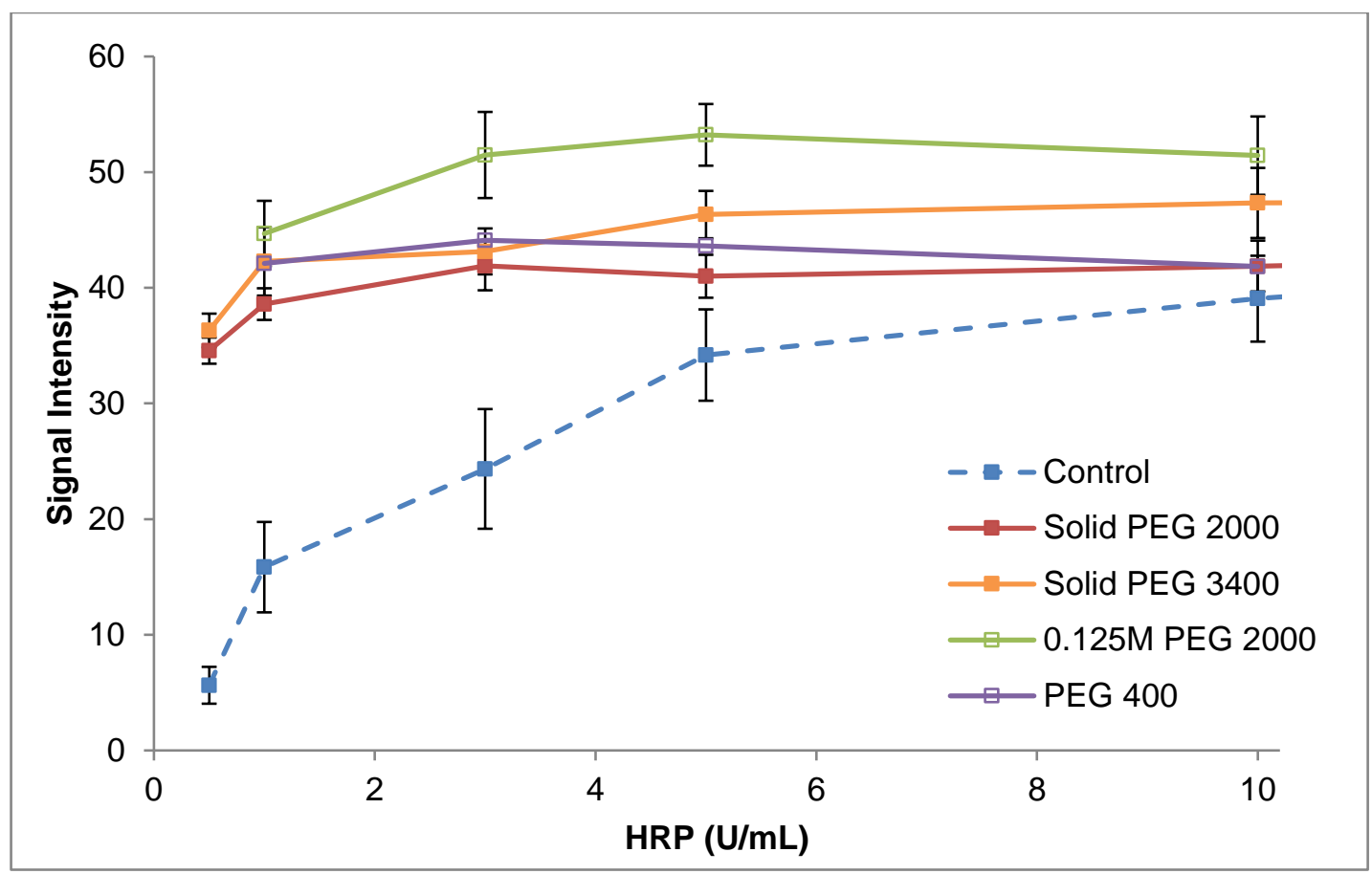

Figure 18. Comparison of HRP activity stabilization in the presence of solid or solution based deposited PEG.

In order to evaluate the stabilizing effect of PEG on HRP at low HRP concentrations, solution deposition of PEG at various molecular weights was observed. Solution deposition provides an advantage in the ability to investigate the effects of PEG at lower molecular weights that are unable to be pressed into a reagent pencil. As mentioned previously, molecular weights below $2000 \mathrm{~g} / \mathrm{mol}$ were deemed too soft to be used as a reagent pencil. With solution deposition, effects of PEG on HRP delivered at 1 $\mathrm{mg}$ of polymer in $2 \mu \mathrm{l}$ volumes onto microPADs was observed in Figure 19A. To achieve this, dilutions were made to achieve the equivalent mass of PEG delivered. This was done to emulate the amount of polymer delivered in a reagent pencil for PEG at lower molecular 
weights unable to be used for solid deposition. Figure 19B shows the results of delivering PEG at a constant concentration ( $2 \mu \mathrm{l}$ of $0.125 \mathrm{M}$ PEG) at varying molecular weights. Interestingly, when the equivalent mass of polymer was delivered, PEG below $400 \mathrm{~g} / \mathrm{mol}$ were not able to stabilize HRP. When the concentration remained constant, PEG 200 was able to stabilize HRP activity slightly, but not as well as its higher molecular weight counterparts. Both results suggest that there is likely a minimum molecular weight required to properly stabilize and reinforce the activity of HRP. 


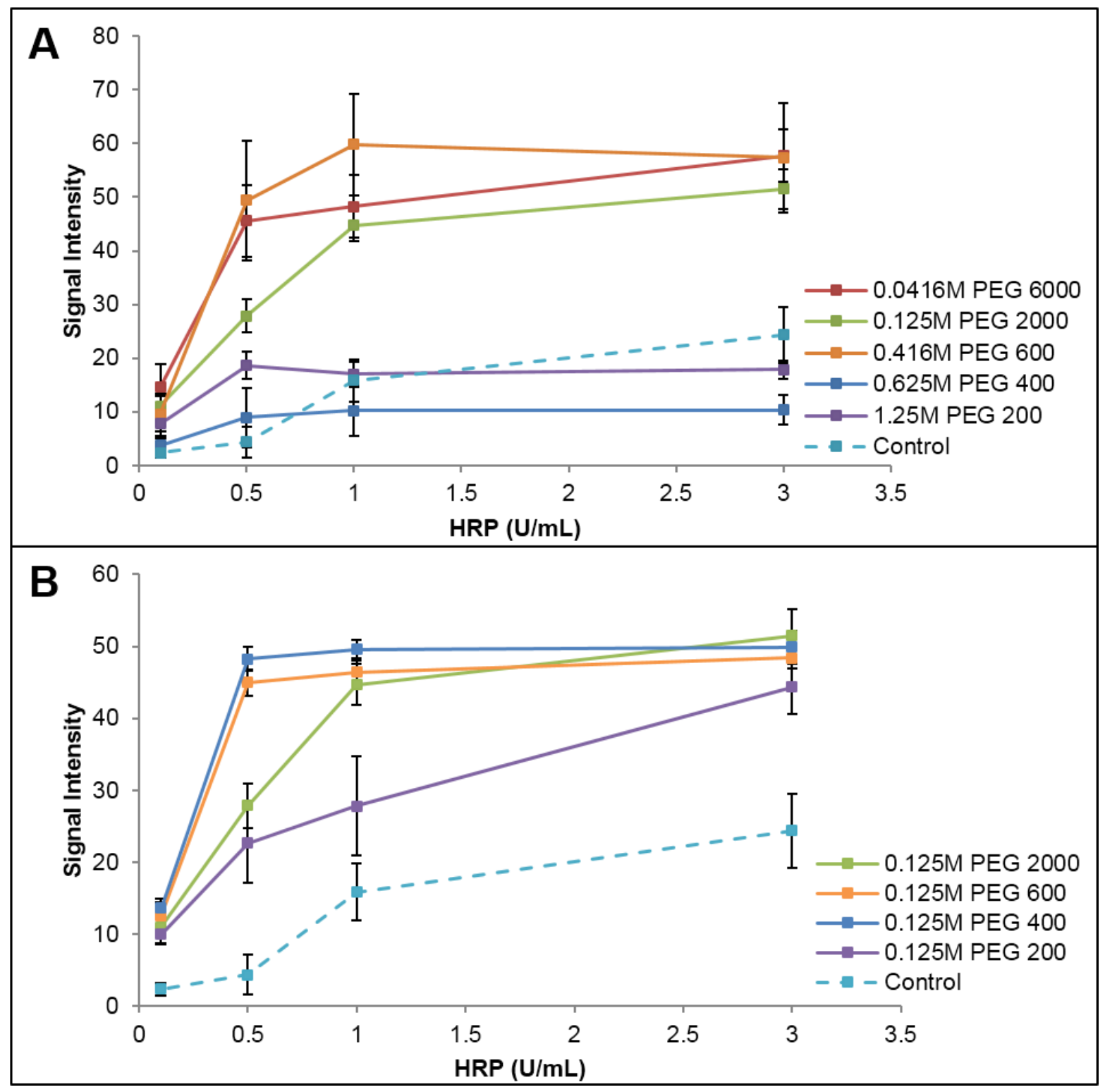

Figure 19. HRP activity in the presence of $A$ ) equivalent mass delivery of $P E G$ and $B$ ) equivalent concentration of solution deposited PEG at varying molecular weights. 


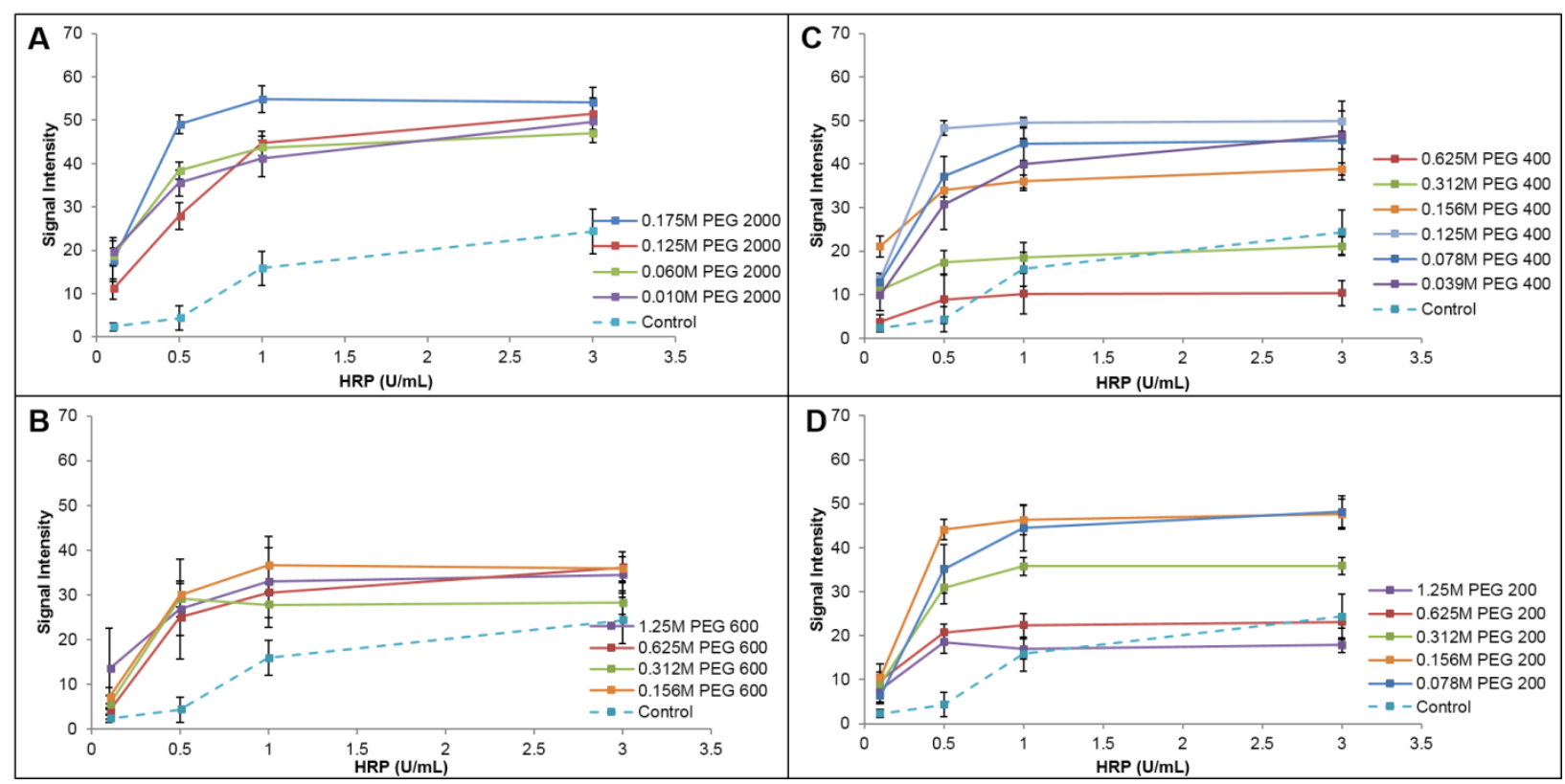

Figure 20. HRP activity in the presence of solution deposited (A) PEG 2000, (B) PEG 600 , (C) PEG 400, and (D) PEG 200 at varying polymer concentrations.

To find the minimum molecular weight at which HRP is stabilized by PEG, molecular weights of $2000,600,400$ and 200 were examined at a range of serial diluted concentrations (Figure 20). Across all concentrations, PEG 2000 reflected the highest HRP signal intensity overall. Although $0.175 \mathrm{M}$ PEG 2000 showed the highest signal intensity at different concentrations of HRP, concentrations of PEG 2000 below $0.175 \mathrm{M}$ showed no significant difference in improving HRP activity (Figure 20A). Further, all concentrations of PEG 600 were also able to improve HRP activity (Figure 20B), but not as well as PEG 2000. Similar to PEG 2000, there was no significant difference in HRP activity relative to the concentration of PEG 600. Finally, PEG 400 and PEG 200 showed differences in their capacity to stabilize HRP as compared to PEG 2000 and PEG 600. 
The presence of PEG 400 had no significant effect on HRP activity at $0.312 \mathrm{M}$ or above (Figure 20C). PEG 200 had no significant effect with concentrations of 0.625 or above (Figure 20D). Below these concentrations, PEG 400 and PEG 200 were able to improve HRP activity as well as PEG 600.

Overall, these results indicate that the molecular weight of PEG is significant in stabilizing HRP, regardless of deposition methodology. High concentrations of lower molecular weight PEG at or below $400 \mathrm{~g} / \mathrm{mol}$ do not significantly improve the activity of HRP at low HRP concentrations. Above $400 \mathrm{~g} / \mathrm{mol}$, there is no correlation between the polymer concentration and relative improvement in HRP activity. In other words, it seems that PEG at any molecular weight above $400 \mathrm{~g} / \mathrm{mol}$ has the capacity to stabilize and improve the activity of HRP, regardless of the polymer concentration.

\subsubsection{ALP Stabilization}

In order to understand the nature of PEG's effects on enzyme stability, it was necessary to investigate the effects of PEG on an enzyme different than HRP. ALP was

chosen for its relative simplicity in assay design and rapid testing. ALP reacts with a BCIP/NBT buffer to yield a purple color whose color intensity can be quantified. Figure 21A shows the preliminary wash tolerance of ALP with and without the presence of PEG 2000. Figure 21B compares the activity of ALP in the presence of solid and solution 
deposited PEG 2000, as well as with solid PEG 3400 and PEG 400, which is a liquid at room temperature.

Overall, the activity of ALP is unaffected by the number of $\mathrm{DI} \mathrm{H}_{2} \mathrm{O}$ washes and remains largely the same across all concentrations of ALP. Solid deposition of PEG also does not significantly affect the activity of ALP. Interestingly, the both solution deposition of $0.125 \mathrm{M}$ PEG 2000 and PEG 400 were able to significantly improve the activity of ALP at low enzyme concentrations. At low concentrations, 0.125M PEG 2000 performed slightly better than PEG 400. In both cases, it appears that the presence of PEG does in fact improve and stabilize the activity of ALP at low enzyme concentrations. 


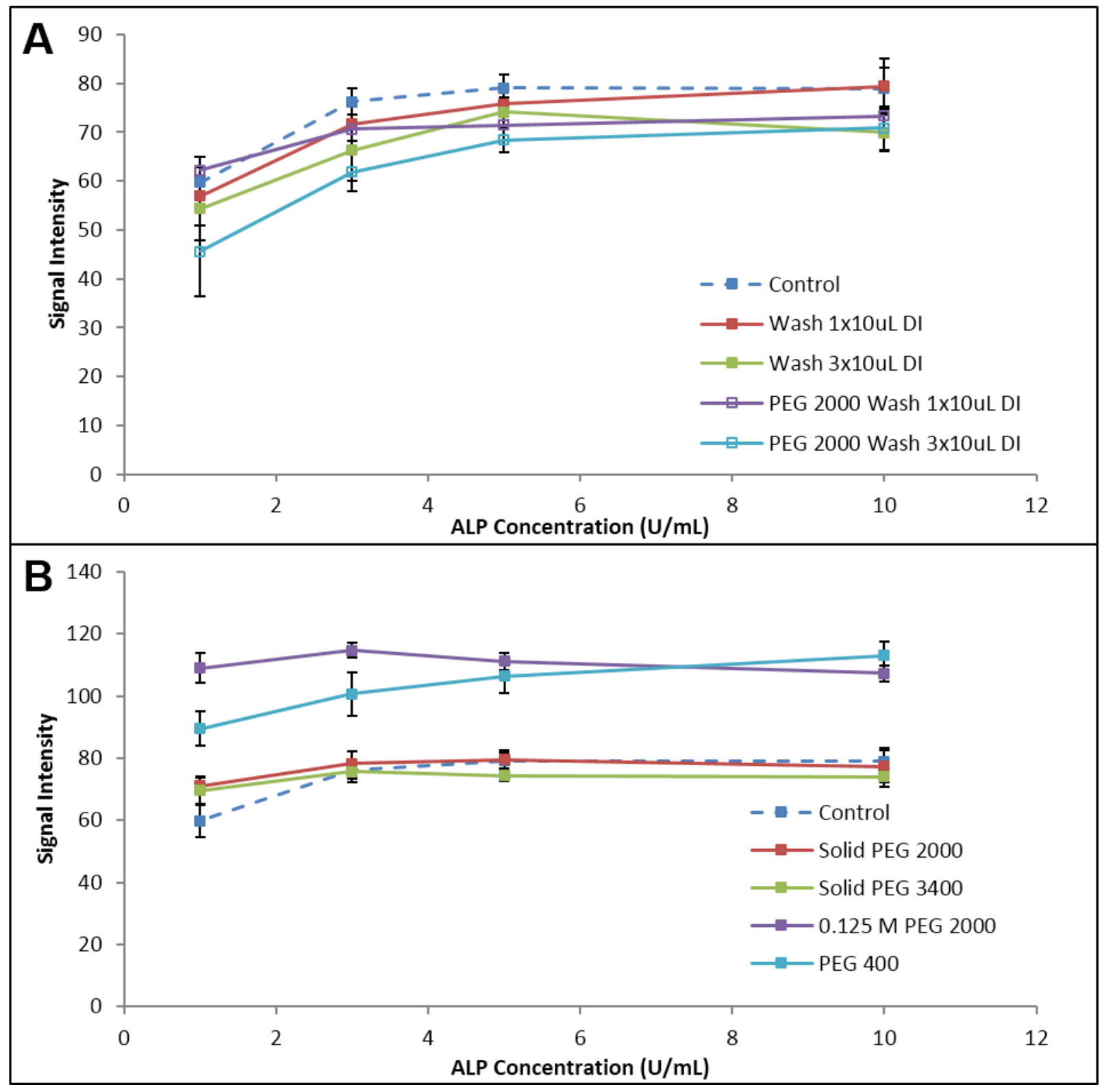

Figure 21. Comparison of ALP (A) wash tolerance and (B) PEG stabilization effects with either solution or solid deposition. 


\subsection{Experimental Methods and Materials}

\subsubsection{Materials}

All reagents, materials, and equipment were purchased from commercial sources unless stated otherwise. The following reagents, materials, and equipment were used: poly(ethylene glycol) $\left(M_{n} \sim 600,2000,3400,6000 \mathrm{~g} / \mathrm{mol}\right.$, Sigma Aldrich), poly(ethylene glycol) methyl ether ( $\mathrm{Mn}_{\mathrm{n}}$ 2000, Sigma Aldrich), poly (ethylene glycol) dimethyl ether $\left(\mathrm{Mn}_{\mathrm{n}}\right.$ 2000, Sigma Aldrich), graphite powder (General's Pure Powdered Graphite), horseradish peroxidase (HRP, 67 U/mg, MP Biomedicals), glucose oxidase (GOx, 266 U/mg, MP Biomedicals), 2,2'-azino-bis(3-ethylbenzothioazoline-6-sulfonic acid) diammonium salt (ABTS, Alfa Aesar), 1-Step ${ }^{\text {TM }}$ ABTS (Thermo Scientific, 1X phosphatebuffered saline (1XPBS pH 7.4, prepared from a 10X solution, Fisher Bioreagents), erioglaucine disodium salt (blue dye, Alfa Aesar), chromatography paper (Whatman No. 1, GE Healthcare Life Sciences), manual pellet press (Parr Instrument Company), analytical balance (Sartorius), flatbed scanner (Epson Perfection V300), automated plot cutter (Graphtec Plotting Cutter CE6000-40), mechanical pencil holders (Art Alternatives), micropipetors (p1000, p200, p20, p10, p2, Gilson), solid ink printer (Xerox Phaser 8560), convection oven(MTI corporation), and a spectrophotometer (Cary 100 UV-vis, Agilent Technologies). 


\subsubsection{Reagent Pencil Fabrication}

\subsubsection{Fabrication of reagent pencils via rotary evaporation}

Reagent pencils were fabricated as shown in Figure 22. For each reagent pencil, a mixture of $75 \%$ polymer and $25 \%$ graphite powder by mass was suspended in acetone. The polymer-graphite matrix could be further customized by adding an additional component of the same polymer at a different molecular weight. These systems were formulated at a 10:90, $75: 25$, or $50: 50$ ratio by mass prior to the addition of graphite and reagent. Reagents were added into the pencil core at varying $\% \mathrm{w} / \mathrm{w}$. The entire mixture was rotary evaporated at $80^{\circ} \mathrm{C}$ to evenly disperse and homogenize the components in the polymer matrix. Once cooled, the mixture was loaded into a manual pellet press and a compressed with a force of about $45 \mathrm{kN}$. This created a cylindrical pellet with either a 6.35 or $3 \mathrm{~mm}$ diameter, depending on the punch size used. These pellets were loaded into a mechanical pencil holder to facilitate reagent deposition onto microPADs. Polymers used include poly(ethylene glycol) (PEG), poly(ethylene glycol) methyl ether (PEGME), and poly(ethylene glycol) dimethyl ether (PEGdiME). Reagents tested include erioglaucine disodium salt (a blue dye), horseradish peroxidase (HRP), glucose oxidase (GOx) and glucose. Pencils were stored in dessicators to prevent softening. 

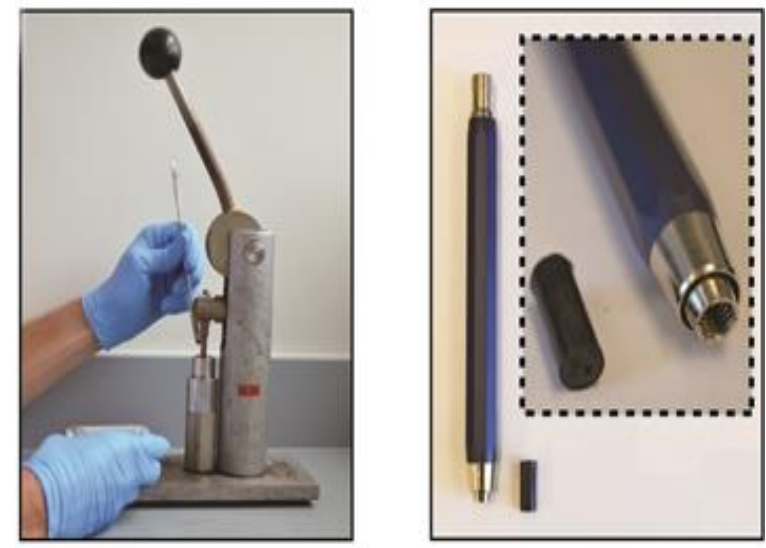

Figure 22. Fabrication of reagent pencils via a manual pellet press to create a 3 or 6.35 $\mathrm{mm}$ diameter pencil core.

2.4.2.2. Fabrication of reagent pencils via lyophilization

The components of each reagent pencil fabricated via lyophilized followed the same composition as reagent pencils fabricated via rotary evaporation. Each polymergraphite or reagent/polymer-graphite mixture was suspended in $7 \mathrm{~mL}$ of $\mathrm{diH}_{2} \mathrm{O}$ and lyophilized for 24 hours. Each mixture was then mixed with a spatula and compressed into a pellet press using the same procedure as reagent pencils fabricated via rotary evaporation. Pencils were stored in dessicators to prevent softening.

\subsubsection{Fabrication of paper-based devices}

MicroPADs were fabricated via wax printing. ${ }^{6}$ Each device pattern was designed on AutoCAD and printed on chromatography paper using a solid-ink printer. The designs used for lateral flow tests is shown in Figure 23. These designs included a 'bone' device with a sample addition zone, a channel, and an end zone as well as a more complex 
'caterpillar' device with multiple zones. The designs used for vertical flow tests are shown in Figure 24. These designs included a '96-well microPAD' design layered on top of blotting paper, and a more complex, folded design that stacks four layers of chromatography paper. For this complex design, the centered circle was punched out to allow for two custom plastic cover to screw into place and ensure even contact between each layer. Each plastic cover was laser cut to leave room at each sample addition zone. For the devices in Figure 23A, Figure 23B, and Figure 24A, chromatography paper with the corresponding device design was placed in a convection oven for 15 minutes at $145^{\circ} \mathrm{C}$ and allowed to cool to room temperature under ambient conditions. For the devices in Figure $3 \mathrm{~B}$, the device was placed in a convection oven for 2 minutes at $195^{\circ} \mathrm{C}$ in order to limit the warpage of that specific device design.
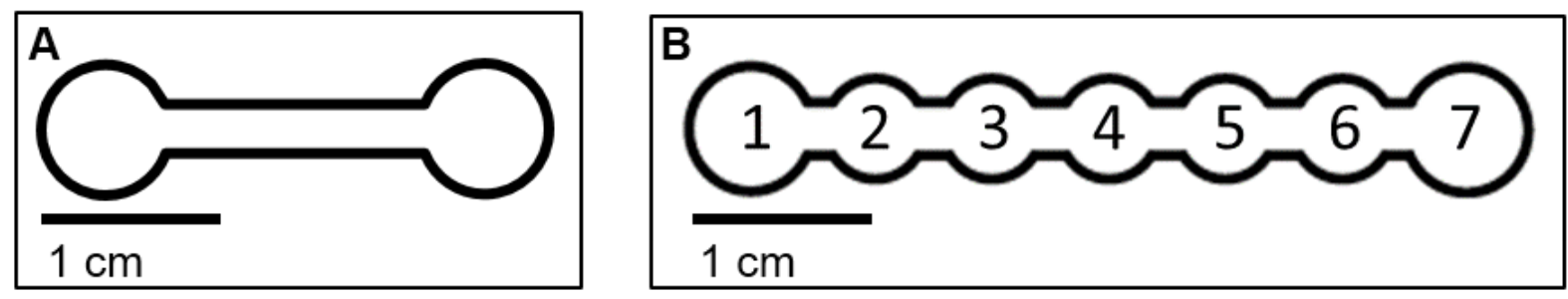

Figure 23. Depiction of (A) simple and B) complex lateral flow device designs used for release profile testing. 

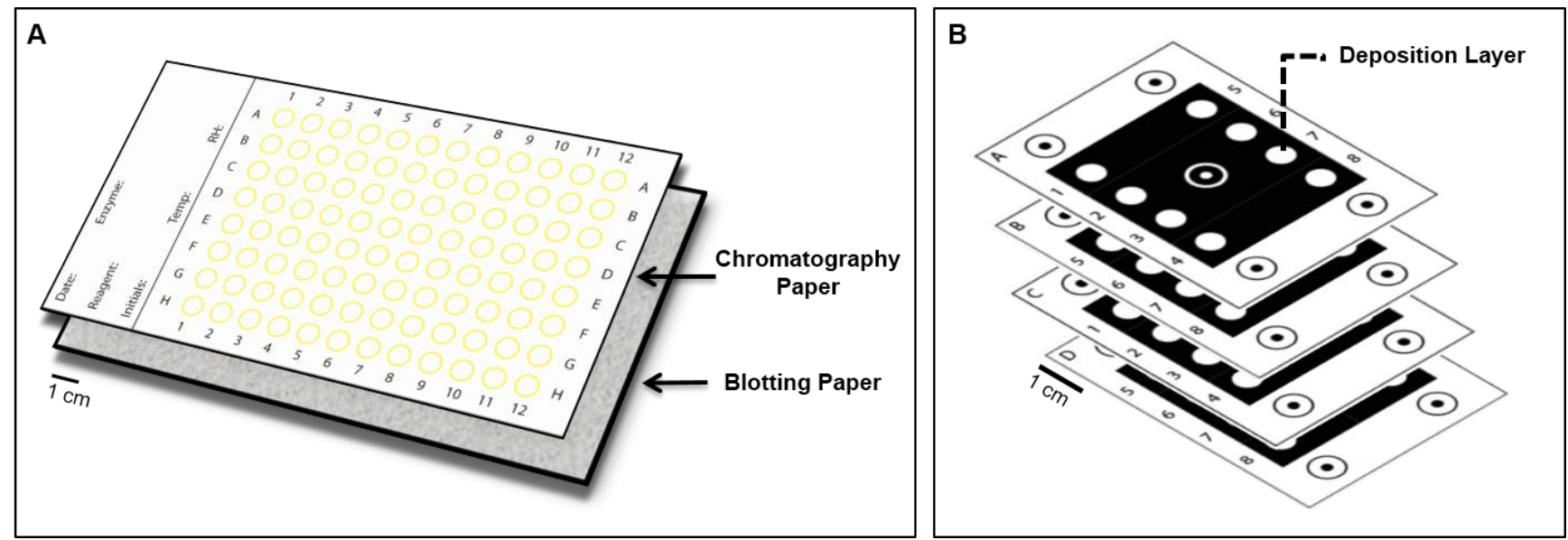

Figure 24. Depiction of $(A)$ simple and $(B)$ complex vertical flow device designs.

\subsubsection{Differential Scanning Calorimetry}

PEG-graphite reagent pencil samples underwent a heat/cool/heat cycle from 0 $100^{\circ} \mathrm{C}$. All samples were tested with a heat flow rate of $10^{\circ} \mathrm{C} / \mathrm{min}$.

2.4.5. Determination of wear for PEG-graphite systems at varying pellet diameters

Preparation of wear resistance testing is depicted in Figure 25. Polymer-graphite systems were varied in composition and pellet diameter and tested for wear resistance. Each pellet was placed in a mechanical pencil holder and loaded perpendicularly onto an automatic plot cutter to apply a constant contact area, force, velocity, and distance traced for each trial. The change in mass for each trial was recorded and wear resistance was calculated as adapted from ASTM G132-96. ${ }^{7}$ Wear was calculated using Equation 1 where $W_{x}$ is the mass loss of the sample, $S_{x}$ is the mass loss of the reference, the mass loss of a PEG 2000-graphite reagent pencil, $\rho$ is the density of the sample $\left(\mathrm{g} / \mathrm{cm}^{3}\right)$, and C is a reference constant $(\mathrm{mg} / \mathrm{mN})$. The reference constant, C, was calculated as the 
mean mass loss $(\mathrm{mg})$ of the reference sample per unit track length $(\mathrm{m})$ per unit load $(\mathrm{N})$. For all samples, the unit track length was $8.17 \mathrm{~m}$, and the load was $2.94 \mathrm{~N}$. The reference reagent pencil of PEG 2000-graphite under these conditions was found to have a mean mass loss of $12.97 \mathrm{mg}$. Each wear test was conducted in 6 replicates.

$$
\text { wear }=\frac{C W_{x}}{\rho S_{x}} m m^{3} / N m
$$

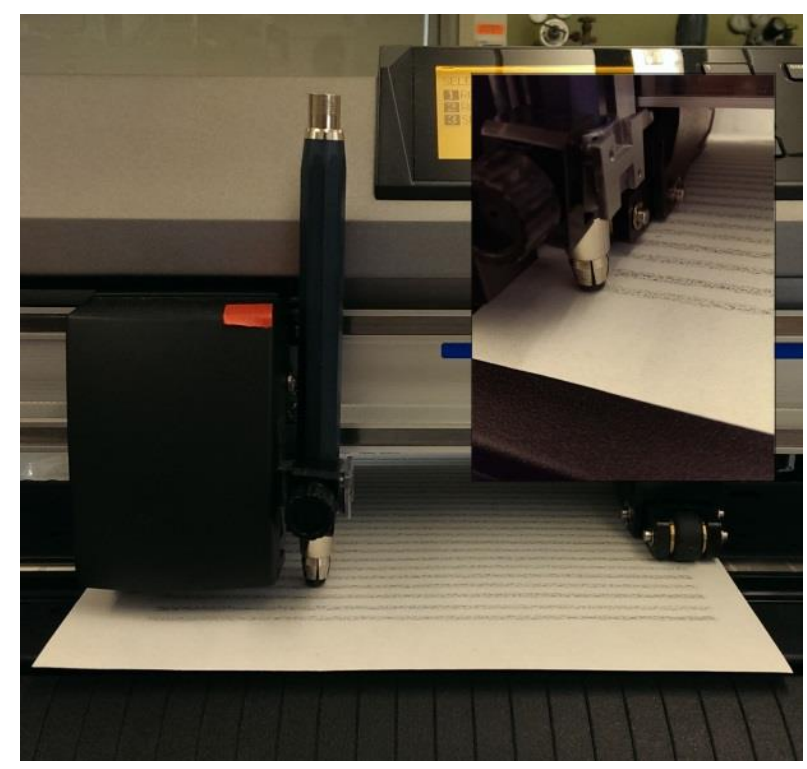

Figure 25. Depiction of wear resistance testing. Pencil cores were loaded onto a pen plotter and deposited reagent at a constant velocity, load, and distance.

\subsubsection{Characterization of blue dye PEG-graphite colorimetric release profile}

Figure 23 depicts the design of the lateral flow microPAD used for release profile testing. Erioglaucine disodium salt was incorporated into a reagent pencil at varying concentrations $(1-15 \% \mathrm{w} / \mathrm{w})$ and used as a model analyte for its colorimetric response to water. The dye was deposited from a fabricated PEG-graphite pencil core onto the 
sample addition zone of a prepared microPAD. Then, the addition of water dissolved the dye into solution and flowed through the hydrophilic channel into a test zone. After 30 minutes, the dried microPADs were scanned and analyzed through Image $1.46 \mathrm{r}$ for colorimetric signal intensity.

\subsubsection{Solution deposition calibration}

\subsubsection{Solution deposition calibration of blue dye.}

A serial dilution of blue dye in deionized water was made $(5,4,3,2,1,0.5,0.25$, $0.125,0.0625,0.03125,0.0156,0.0078$, and $0.0039 \mathrm{mM}$ blue dye). $13 \mu \mathrm{l}$ of each dilution was delivered to a suspended lateral flow device as pictured in Figure 2 in 10 replicates. The device was allowed to dry for 30 minutes before the image was scanned.

\subsubsection{Solution deposition calibration of glucose}

A serial dilution of glucose in deionized water in $1 \mathrm{~mL}$ aliquots was made $(5,4,3$, $2,1,0.75,0.50,0.25$, and $0.10 \mathrm{mM}$ glucose). $11 \mu \mathrm{l}$ of each dilution was delivered to the sample addition zone of a suspended lateral flow device as pictured in Figure 2. The device was allowed to dry for 5 minutes before $2 \mu$ of a HRP/GOx/ABTS solution was added to the end zone. The device was allowed to dry for 30 minutes before the device was scanned and analyzed in ImageJ. Tests were conducted in 10 replicates for each dilution. 


\subsubsection{Solution deposition calibration of horseradish peroxidase}

A serial dilution of HRP in 1XPBS in $1 \mathrm{~mL}$ aliquots was made $(20,10,5,3,2,1$, and $0.5 \mathrm{U} / \mathrm{mL}$ HRP). $2 \mu$ of each dilution was delivered to the a sample zone in a paper based 96 -well plate in 10 replicates. The solution was allowed to dry for 5 minutes before $1 \mu \mathrm{l}$ of a 1 Step ABTS solution was added to the end zone. The device was allowed to dry for 30 minutes before the image was scanned and analyzed in ImageJ. Tests were conducted in 10 replicates for each dilution.

\subsubsection{Reagent Pencil Release Efficiency}

\subsubsection{Blue dye and glucose reagent pencil efficiency}

The change in mass was measured for the deposition of reagent pencil samples on a lateral flow device. Each reagent pencil sample was run analogously to their respective solution deposition samples. For samples of blue dye, $13 \mu$ of deionized water was pipetted on top of the sample addition zone. 10, 7.5, and $5 \% \mathrm{w} / \mathrm{w}$ blue dye PEGdiME reagent pencils were tested using this methodology. For glucose samples, $11 \mu \mathrm{l}$ of deionized water was added to the sample addition zone and allowed to dry for 5 minutes before the subsequent addition of $2 \mu \mathrm{l}$ of HRP/GOx/ABTS solution. $7.5,6.3,5,3.2$, and $1.3 \% \mathrm{w} / \mathrm{w}$ glucose PEGdiME reagent pencils and $6.3,5,3.2$, and $1.3 \% \mathrm{w} / \mathrm{w}$ PEG reagent pencils were tested using this methodology. All devices were allowed to dry for 30 minutes after the last addition step before scanning and analyzing the device in ImageJ. Changes 
in deposition mass were correlated to the signal intensity in the end zone of each device. Tests were conducted in 10 replicates for each reagent pencil tested.

2.4.8.2. Horseradish peroxidase reagent pencil efficiency.

The change in mass was measured for the deposition of reagent pencil samples on a paper based 96 well plate. Each reagent pencil sample was run analogously to their respective solution deposition samples. $1 \mu \mathrm{l}$ of 1 step ABTS was added on top of the deposited HRP and samples were allowed to dry for 30 minutes before the device was scanned and analyzed in ImageJ. 6.3, 5, and 3.2\% w/w HRP PEGdiME reagent pencils were tested using this methodology. Tests were conducted in 10 replicates.

\subsubsection{PEG stabilization of HRP and ALP}

All HRP and ALP stabilization experiments were conducted on 96 well microPADs. Serial dilutions of PEG 2000, 600, 400, and 200 were made in $\mathrm{DI}_{2} \mathrm{O} .5 \mathrm{~mL}$ of $0.175 \mathrm{M}$ PEG 2000 (1.7540g, $0.877 \mathrm{mmol}$ ) was serial diliuted to create $1 \mathrm{~mL}$ aliquots of $0.125 \mathrm{M}$, $0.060 \mathrm{M}$, and 0.010M PEG 2000 dilutions. $1 \mathrm{~mL}$ of $1.25 \mathrm{M}$ PEG $600(0.7531 \mathrm{~g}, 1.25 \mathrm{mmol})$ was serial diluted to create $0.5 \mathrm{~mL}$ aliquots of $1.25 \mathrm{M}, 0.625 \mathrm{M}, 0.312 \mathrm{M}$, and $0.156 \mathrm{M} \mathrm{PEG}$ 600 dilutions. $1 \mathrm{~mL}$ of $0.625 \mathrm{M}$ PEG $400(0.2 \mathrm{~mL}, 0.56 \mathrm{mmol})$ was serial diluted to create $0.5 \mathrm{~mL}$ aliquots of $0.625 \mathrm{M}, 0.312 \mathrm{M}, 0.156 \mathrm{M}, 0.125 \mathrm{M}, 0.078 \mathrm{M}$, and $0.039 \mathrm{M}$ PEG 400 dilutions. $5 \mathrm{~mL}$ of $1.25 \mathrm{M}$ PEG $200(1.11 \mathrm{~mL}, 6.25 \mathrm{mmol})$ was serial diluted to create $1 \mathrm{~mL}$ aliquots of $1.25 \mathrm{M}, 0.625 \mathrm{M}, 0.312,0.156 \mathrm{M}$, and $0.078 \mathrm{M}$ PEG 200 dilutions. 
Enzyme solutions were created as follows. HRP solutions utilized were serial diluted to $1 \mathrm{~mL}$ aliquots of $20,10,5,3,1,0.5$, and $0.1 \mathrm{U} / \mathrm{mL}$ in $1 \times P B S$ buffer, as described previously. $10 \mathrm{~mL}$ of $10 \mathrm{U} / \mathrm{mL}$ ALP $(3.84 \mathrm{mg}, 0.384 \mathrm{mg} / \mathrm{mL})$ was serial diluted in $1 \times$ Tris buffer $(\mathrm{pH} 7.4$ ) to create $1 \mathrm{~mL}$ aliquots of $10,5,3$, and $1 \mathrm{U} / \mathrm{mL}$ ALP dilutions.

\subsubsection{PEG stabilization of HRP}

When controlling for equivalent mass $(1 \mathrm{mg})$ delivered to the microPAD, concentration of respective molecular weight PEGs were varied. For this specific experiment, the signal intensities of HRP in the presence of $0.0416 \mathrm{M}$ PEG $6000,0.125 \mathrm{M}$ PEG 2000, 0.416M PEG 600, 0.625M PEG 400, and 1.25M PEG 200 were compared. When comparing concentration dependence, equivalent dilutions of each molecular weight PEG was compared.

Both solid and solution deposition of PEG was investigated. For solution deposition, $4 \mu \mathrm{l}$ of PEG solution was pipetted into an addition zone of a suspended 96 well microPAD and allowed to dry for 30 minutes. For solid deposition, a pure PEG reagent pencil, without a graphite component, was drawn onto the sample addition zone of the microPAD. After drying, $2 \mu \mathrm{L}$ of HRP and $1 \mu \mathrm{L}$ of 1 step ABTS were pipetted over the dried PEG. The microPAD was allowed to dry for 30 minutes before the device was scanned and analyzed in ImageJ software. All samples were run with 10 replicates. All HRP samples were analyzed in the red color channel in ImageJ. 


\subsubsection{PEG stabilization of ALP}

For ALP, both solid and solution deposition of PEG was investigated. For all ALP stabilization tests with solution deposition, $4 \mu$ of PEG solution was pipetted onto an addition zone of a suspended 96 well microPAD and allowed to dry for 30 minutes. Samples with solid deposition of PEG were deposited with pure PEG reagent pencils without graphite. Then, $10 \mu \mathrm{L}$ of $\mathrm{DI} \mathrm{H}_{2} \mathrm{O}$ washes were pipetted over the dried PEG prior to the addition of ALP and allowed to dry for another 30 minutes for samples with wash steps involved. After drying, $2 \mu \mathrm{L}$ of ALP solution and $3 \mu \mathrm{L}$ of BCIP/NBT phosphate for membrane buffer was pipetted over the dried PEG. The microPAD was allowed to dry for 30 minutes before the device was scanned and analyzed in ImageJ software. All samples were run with 10 replicates. All ALP samples were analyzed in the green color channel in ImageJ. 
CHAPTER 3. MANIPULATION OF PNIPAM LCST FOR FLUID FLOW MANIPULATION

In an effort to expand the utility of reagent pencils and microPADs for POC diagnostics, alternative polymers to PEG were investigated for their stimuli responsive characteristics. If reagent pencils can incorporate stimuli responsive qualities after their delivery onto microPADs, the versatility and complex design of microPADs can improve, granting greater relevance to paper microfluidics in the POC field.

\subsection{Thermoresponsive Polymers and Click Chemistry}

\subsubsection{Characteristics of Thermoresponsive Polymers and Poly( $\mathrm{N}$-isopropylacrylamide)}

Thermally responsive materials and their characterization find importance in many interdisciplinary fields, such as drug delivery systems or self-healing materials. ${ }^{20}$ In thermally responsive polymers, miscibility of polymer in solution is temperature dependent.

Certain polymers reach a critical solution temperature at which they become miscible or immiscible in solution, depending on the critical temperature reached. Many polymers of interest with critical solution temperatures exist, including poly $(\mathrm{N}$ isopropylacrylamide) (PNIPAM), other N-substituted acrylamides, poly(ethylene oxide), poly(vinyl methyl ether), among others. ${ }^{21}$ Below the upper critical solution temperature (UCST), a polymer is immiscible. When heated above the UCST however, the polymer solution becomes soluble. The opposite trend is true for the lower critical solution 
temperature (LCST). Below the LCST, a polymer solution is miscible. If heated above the LCST, the polymer becomes immiscible. Polymers can exhibit either one or both UCST and LCST. ${ }^{22}$ Figure 26 depicts an example of a polymer with both a UCST and LCST. This figure shows that, below the UCST and above the LCST, polymer exists in a phase separated region. Conversely, above the UCST and below the LCST, the polymer exists in a single phase region where it is miscible. The region between the bimodal and spinodal curves exists a metastable region, where the polymer is partially soluble in solution. Polymer solubility can be modified according to various characteristics including a change in solvent composition, the introduction of a cosolvent, polymer molecular weight and composition. The ability to thermally control polymer solubility is extremely useful in a wide range of applications, especially in the case of controlled release drug delivery systems. 


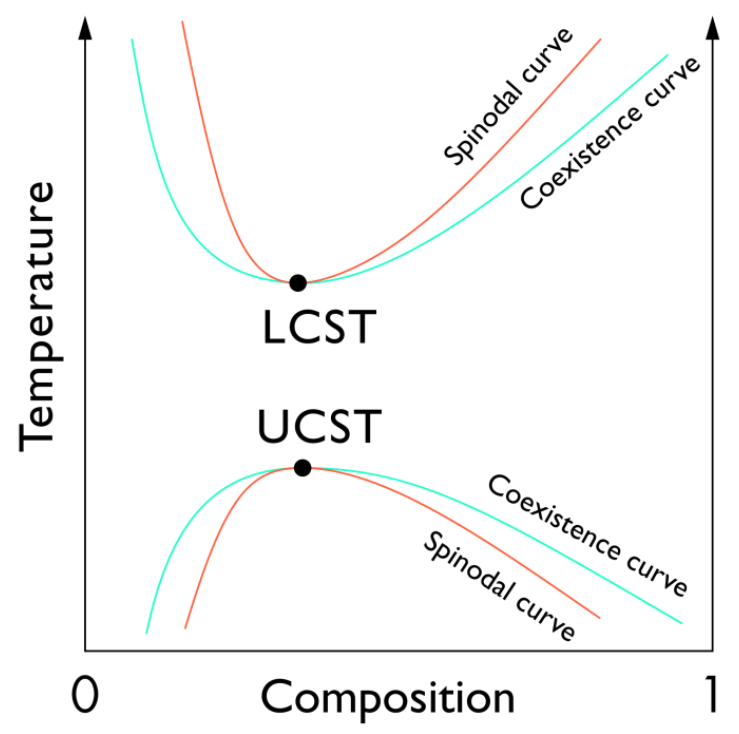

Figure 26. Phase diagram for a polymer blend that has both a UCST and LCST. The Coexistence curve is also known as the binodal curve. ${ }^{16}$

This work widely focuses on the use of PNIPAM and the manipulation of its phase transition. The solubility of PNIPAM is temperature dependent and has been found to have a LCST at $32^{\circ} \mathrm{C}$ in water. ${ }^{21}$ Below the LCST, the solubility of PNIPAM is dictated by hydrogen bonding between the polymer and solvent. PNIPAM experiences a change in solubility when heated above its LCST, where temperature agitation provides kinetic energy for vibrational and rotational motion in isopropyl groups. This effectively breaks up hydrogen bonding and releases solvent molecules trapped within the polymer matrix, leading to the insolubility of PNIPAM. Figure $\mathbf{2 7}$ below depicts a computational model of PNIPAM both above and below its LCST in water. PNIPAM has been used extensively in applications for drug release because of its favorable LCST near physiological 
temperatures. Further, because of its roughly similar structure to proteins, PNIPAM has also been used as a crude protein-model polymer.

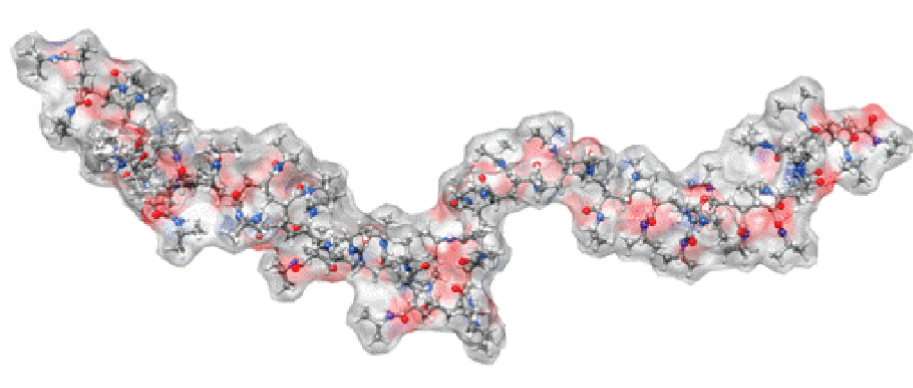

$a$

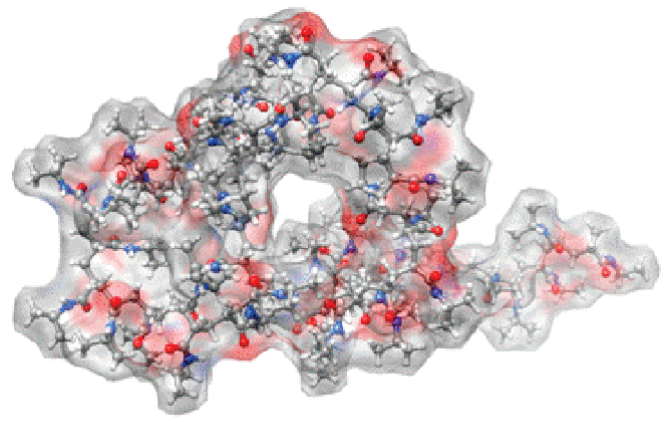

$b$

Figure 27. PNIPAM conformation from a molecular dynamic simulation at (A) $295 \mathrm{~K}$ and (B) $310 \mathrm{~K} .{ }^{23}$

There have been numerous efforts and methods made to manipulate the LCST of PNIPAM. These methods include copolymerization, molecular weight change, and salt concentration. It has been noted that copolymerization of PNIPAM can sacrifice the capacity of the polymer's controlled release. ${ }^{24}$ The variance of salt composition and concentration manipulates the LCST of PNIPAM by taking advantage of the salting out effect. Depending on the strength of the cation-anion pair and its ability to disrupt the solvating power of water, it has been shown that the LCST of PNIPAM can be lowered by a significant amount. Roughly, the relative ability of salts to manipulate the LCST of PNIPAM follows the Hofmeister series, which typically is used to evaluate the ability for ions to precipitate proteins from an aqueous solution. In this series, $\mathrm{Na}_{2} \mathrm{CO}_{3}>\mathrm{Na}_{2} \mathrm{SO}_{4}>$ 
$\mathrm{Na}_{2} \mathrm{~S}_{2} \mathrm{O}_{3}>\mathrm{NaH}_{2} \mathrm{PO}_{4}>\mathrm{NaF}>\mathrm{NaCl}>\mathrm{NaClO}_{4}>\mathrm{NaBr}>\mathrm{NaNO}_{3}>\mathrm{Nal}>\mathrm{NaSCN}$ where the salts furthest to the right represent the most destabilizing and weakly hydrated salts.

PNIPAM is of special interest for its use in microPADs. As a stimuli responsive polymer, if it can be incorporated into a reagent pencil or grafted onto the paper substrate, it may be possible to create a more sophisticated microPAD with a selective polymer barrier. The investigation of PNIPAMs possible incorporation into microPADs, and ultimately reagent pencils, is of significant interest in this work.

\subsubsection{Thiol-ene Click Chemistry}

In the early 2000's, Sharp et al. proposed a class of reactions, deemed 'click' reactions, that produced high yield stereospecific and selective products in mild conditions. Typically, click chemistry was developed with a focus on the generation of stereospecific carbon-carbon bonds. The simplicity of click chemistry refined the conventional process for chemical syntheses that typically required extensive protecting group strategies. Further, characteristic click reactions produce easily removable byproducts, if any, and are relatively insensitive to oxygen or water. Notable reaction families of click chemistry reactions include azide-alkyne cycloaddition, thiol-ene addition, thiol-yne and thiol-isocyanate reactions, Diels Alder addition of dienes to dienophiles, 1,3 dipolar cycloadditions, and oxime/hydrazone formation. ${ }^{25}$ Thiol-ene click chemistry is of particular interest for this work. Thioether products form upon the generation of thiol 
radicals from introduction of light or heat. In the case of multifunctional thiols and enes, it is possible to create cross-linked materials with high conversions.

In an effort to investigate whether or not a stimuli responsive reagent pencil was viable, PNIPAM was chosen for its LCST characteristics and potential incorporation into microPAD design. To flesh out PNIPAM's viability as a reagent pencil, it is necessary to understand its performance as a reagent pencil as compared to PEG. If PNIPAM performs satisfactorily as the polymer component of reagent pencils, it becomes possible to investigates its utility as a stimuli responsive component of microPADs.

\subsubsection{Experimental Plan and Overview}

PNIPAM was specifically chosen as a potential polymer component as a reagent pencil particularly for its stimuli responsive capacity. If PNIPAM can be properly incorporated as a reagent pencil, manipulation of PNIPAM's solubility on paper via LCST manipulation can be used to act as a 'gate' for fluid flow. If PNIPAM is to be used as a reagent pencil, its mechanisms for wear, release profile, and release efficiency must first be understood. If PNIPAM performs satisfactorily, it becomes possible to investigate its use for fluid flow manipulation both laterally and vertically through microPADs. In particular, different salts and salt concentrations will be investigated to lower the LCST of PNIPAM below room temperature. The successful implementation of PNIPAM in microPADs will allow for more complex microPAD development. In other words, a 
PNIPAM reagent pencil would act as a novel tool available in the field of paper microfluidics that will work to complement other reagent pencil tools available.

Of related interest, this work also investigates the use of thiol-ene click chemistry with microPADs. Chromatography paper can be functionalized with alkenes by reacting available hydroxyl groups with acryloyl chloride in DMF. Alkene functionalized paper can be used to graft thiol terminated (R-SH) reagents onto the paper via photoinitiated thiolene click chemistry. This can include thiol-terminated polymers, small molecules, or proteins with available cysteine groups on its surface. As before, these reagents can also be incorporated into reagent pencils and drawn onto microPADs. Figure 28 below summarizes the synthetic scheme for the functionalization of chromatography paper and the subsequent grafting of $\mathrm{R}-\mathrm{SH}$ reagents.

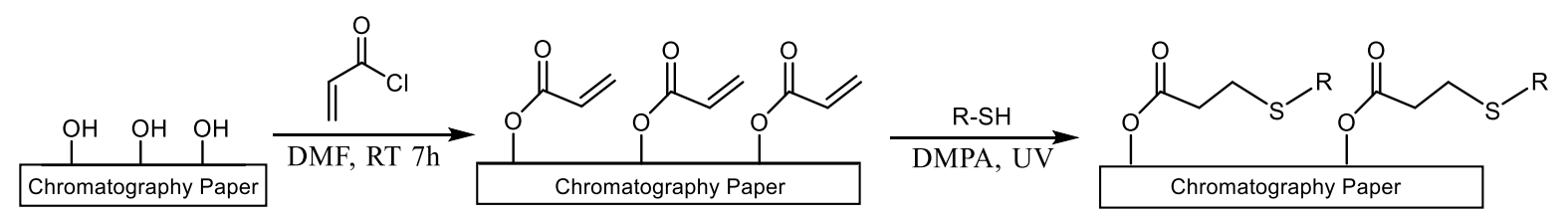

Figure 28. Scheme of chromatography paper functionalization via photoinitiated thiolene click chemistry.

The characteristic of grafting reagents can be advantageous for several reasons.

First, the functionalization process itself is simple and relatively inexpensive. This does not compromise the effort to provide low-cost, efficient diagnostic assays to POC locations. Further, this process lends itself to novel applications in the design of microPADs. If a protein or signaling molecule of interest is able to covalently bind to the 
surface, signal dilution from washing steps can be reduced. In this respect, it may be possible to achieve greater reagent sensitivity and signal range in developed diagnostic assays. Potentially, improving wash tolerance may also lead to the development of reusable microPADs. In another case, if a stimuli responsive polymer (eg. PNIPAM) is grafted, the manipulation of its LCST allow for the development of selective 'gates' that can be incorporated into device design.

\subsection{Characterization of PEG/PNIPAM Reagent Pencil Systems}

The incorporation of PNIPAM into reagent pencils was first investigated for its wear, release profile, and release efficiency characteristics in the same fashion as PEG based reagent pencils were evaluated previously. The evaluation of PNIPAM's performance as a reagent pencil is necessary before it can be considered for use as a stimuli responsive tool in microPAD design. For the reagent pencil and fluid flow portion of this work, a $3.76 \times 10^{3} \mathrm{~g} / \mathrm{mol}$ nitrile and dithiobenzene terminated PNIPAM was utilized. A $1.603 \times 10^{4}$ $\mathrm{g} / \mathrm{mol}$ RAFT polymerized thiol terminated PNIPAM was also utilized for the fluid flow aspect of this work. Both structures and corresponding GPC traces are shown in Figure 29 below. Table 2 summarizes their molecular weight and polydispersity characteristics.

Scheme 1. Synthesis of thiol terminated PNIPAM

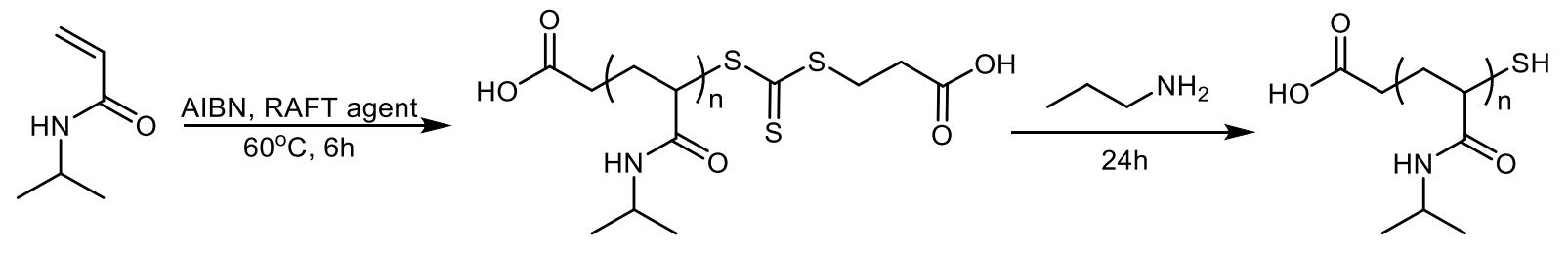




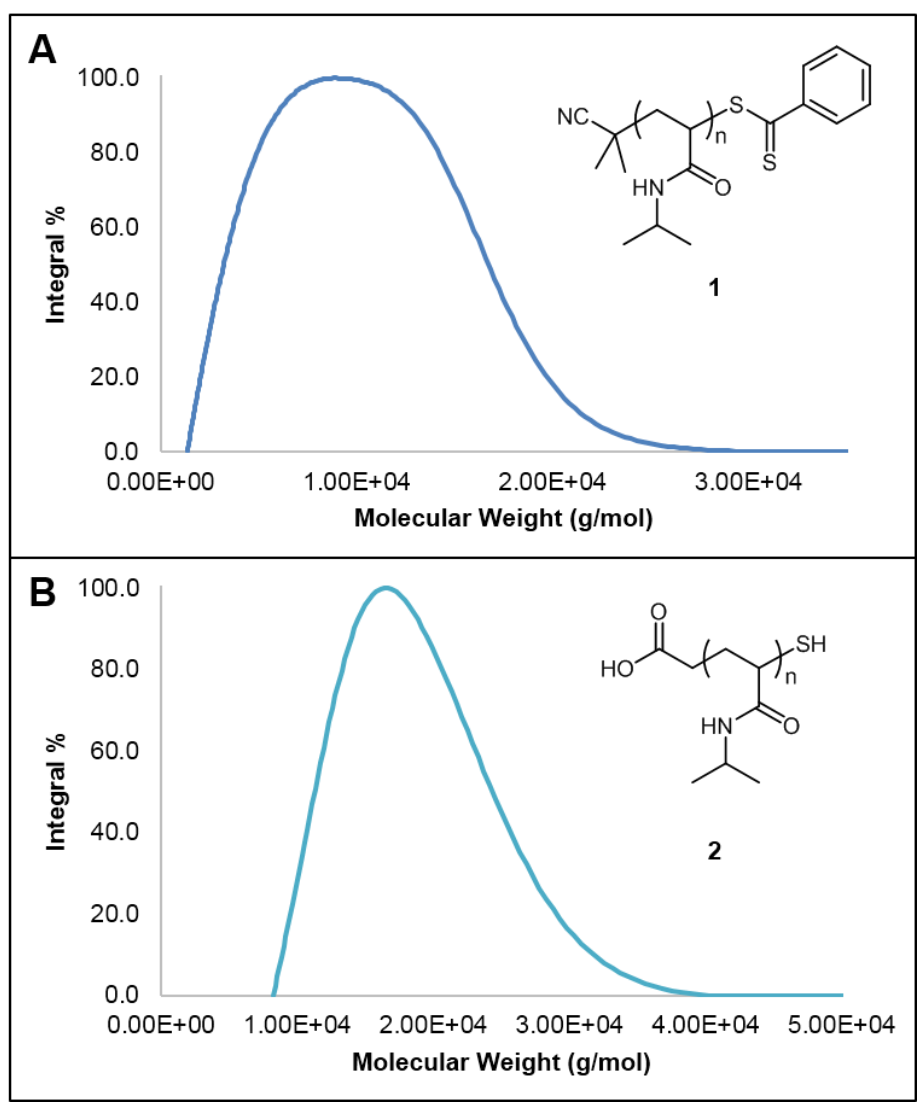

Figure 29. GPC traces and corresponding structure for $(A)$ nitrile and dithiobenzene terminated PNIPAM and $(B)$ thiol terminated PNIPAM.

Table 2. PNIPAM GPC characteristics.

\begin{tabular}{cccc}
\hline PNIPAM Sample & $\mathbf{M}_{\mathbf{n}} \mathbf{( \mathbf { g } / \mathbf { m o l } )}$ & $\mathbf{M}_{\mathbf{w}}(\mathbf{g} / \mathbf{m o l})$ & PDI \\
\hline 1 & 3765 & 7123 & 1.892 \\
\hline 2 & 16034 & 17419 & 1.086
\end{tabular}

3.2.1. Effect of graphite variance and polymer on wear, colorimetric release profile, and release efficiency

Because the initial release efficiency tests demonstrated that efficiency is independent of different PEG iterations, both pNIPAM $\left(\mathrm{M}_{\mathrm{n}}=3765 \mathrm{~g} / \mathrm{mol}\right)$ reagent pencils 
and reagent pencils with varying graphite content was tested on lateral flow microPADs to determine their relation to release efficiency. For comparison, iterations of PEGdiME systems were also tested. pNIPAM was chosen as a polymer for comparison because of its brittle nature when pressed, not dissimilar to the brittle attributes of PEG 6000. Further, as a thermoresponsive polymer, PNIPAM has the potential for the development of other applications and uses for microPADs. At a glance, Figure $\mathbf{3 0}$ compares the wear of PEG, PEGME, PEGdiME, and pNIPAM reagent pencils with no additional components. This plot shows that pNIPAM has the lowest wear compared to other PEG variations. Figure 31 shows the results for the wear, colorimetric release profile, and release efficiency for reagent pencil systems of $5 \%$ blue dye w/w PNIPAM and PEGdiME with varying graphite content. Overall, decreasing graphite content led to an increase in wear for PEGdiME systems while pNIPAM systems saw no change in wear regardless of graphite composition. The colorimetric release profiles for both PEGdiME and pNIPAM reagent pencils remained constant regardless of graphite content. Overall, the release profiles of PEGdiME systems were significantly higher than the release profiles of pNIPAM based systems despite both having a $5 \% \mathrm{w} / \mathrm{w}$ blue dye composition. This suggests that the release of blue dye from pNIPAM systems is less efficient than PEGdiME systems. Indeed, when examining the release efficiency of these systems, it is clear that graphite content has no significant sway on release efficiency and that pNIPAM systems are less efficient than PEGdiME systems. 


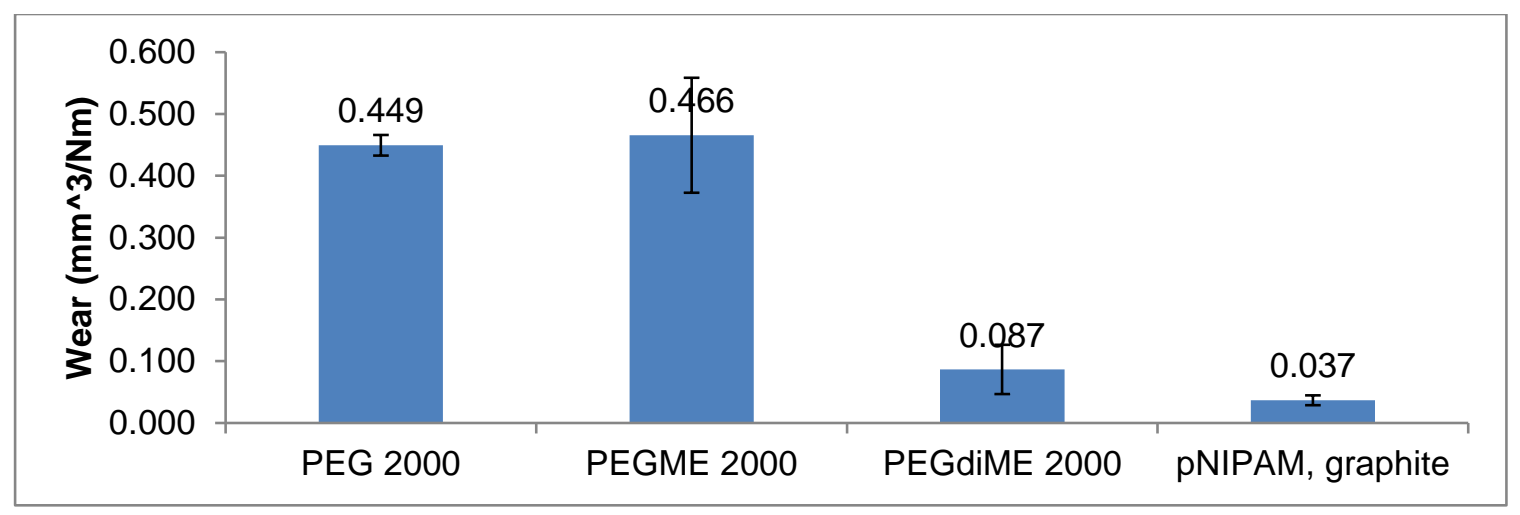

Figure 30. pNIPAM vs PEG variants in wear. no BD incorporated.

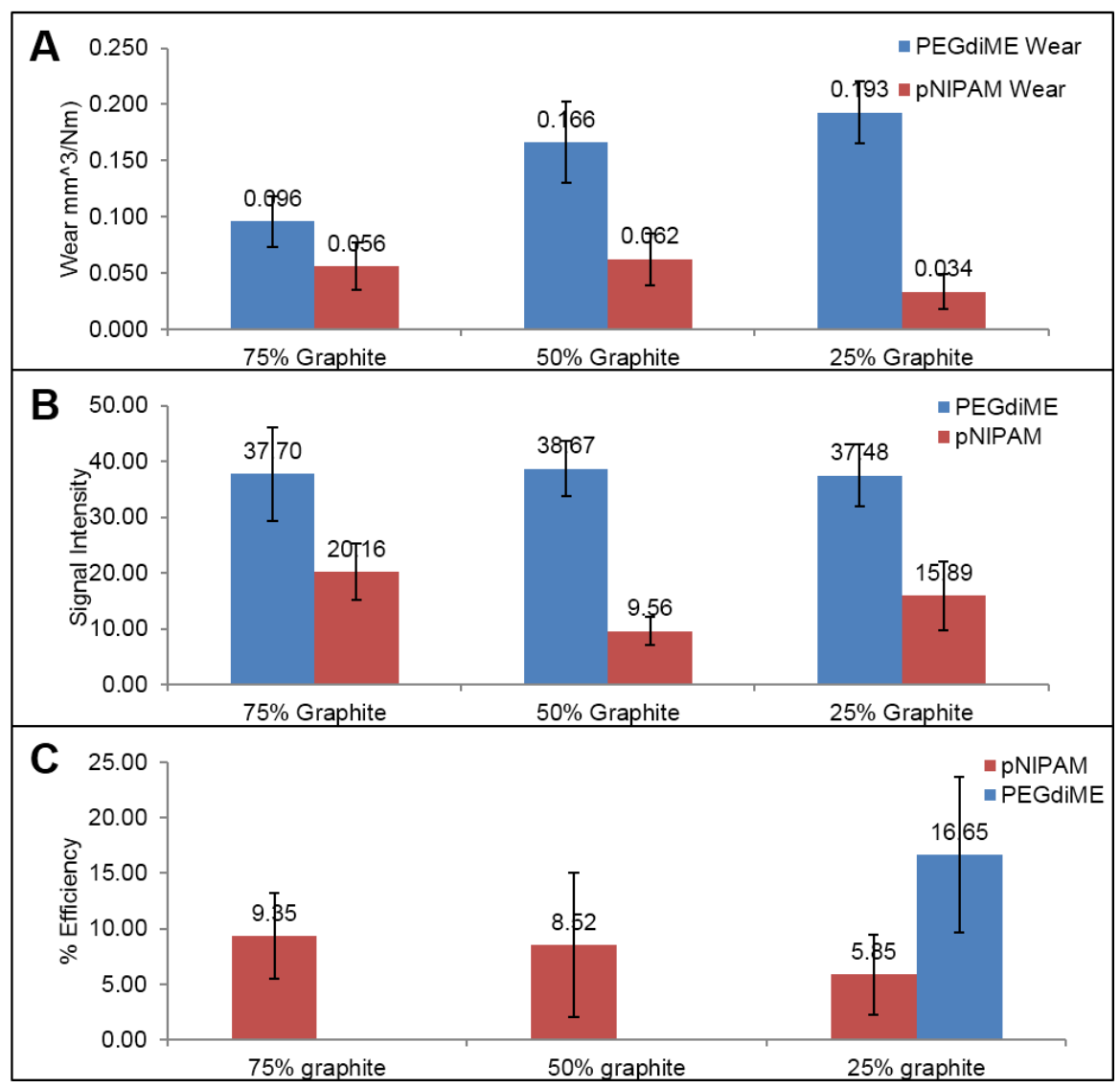

Figure 31. Trends in $(A)$ wear and $(B)$ release profile for varying graphite concentration at constant w/w 5 wt \% blue dye in PEGdiME 2000 and pNIPAM. (C) shows the release efficiency of pNIPAM samples with varying graphite concentration. 
3.2.2. Effect of PEG on pNIPAM for wear, colorimetric release profile, and release efficiency

Because pNIPAM systems were shown to be more inefficient than PEG based systems, new pNIPAM/PEG reagent pencils were formulated at 25:75, 50:50, and 75:25 ratios with $5 \% \mathrm{w} / \mathrm{w}$ blue dye in an effort to improve their efficiency on lateral flow microPADs. Figure 32 shows the results for the wear, colorimetric release profile, and release efficiency for these pNIPAM/PEG systems. Overall, the increasing PEG incorporation into pNIPAM reagent pencils saw an increase in wear (Figure 32A). Similar to before, the colorimetric release profile was not significantly different across these systems (Figure 32B), however the release efficiency saw a notable improvement with increased PEG incorporation (Figure 32C). This experiment demonstrated the customizable efficiency of reagent pencil systems to accommodate for different systems. By combining a brittle polymer such as pNIPAM with a known, softer polymer matrix, such as PEG, the release efficiency of reagents can be improved. Because it is viable to incorporate a wider range of polymers into the reagent pencil, it may be possible to investigate the stimuli responsive features of other polymer systems, such as temperature responsiveness in pNIPAM, and their potential applications on microPADs. 


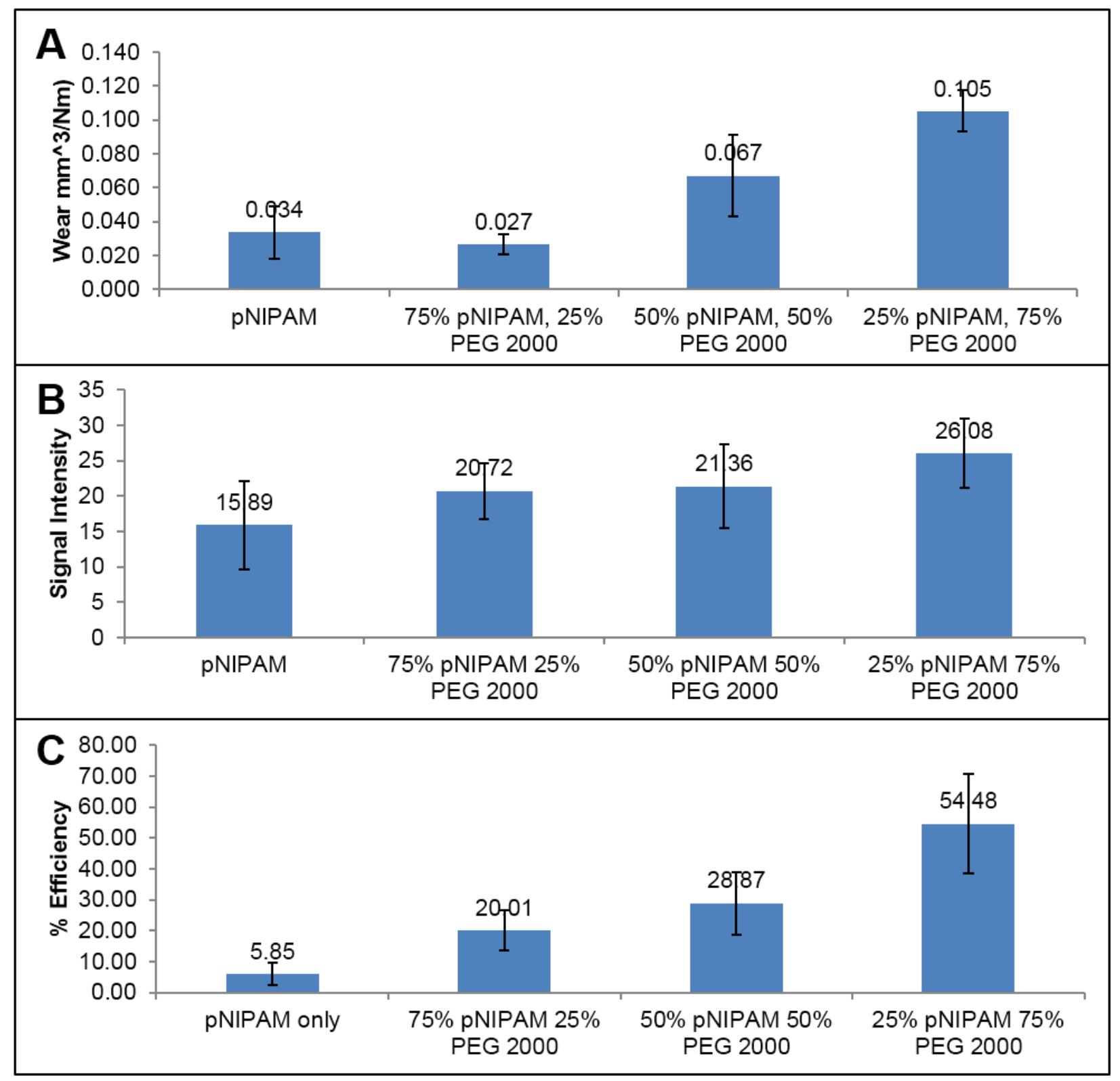

Figure 32. Trends in (A) wear, $(B)$ release profile and $(C)$ release efficiency for pNIPAM-PEG 2000 systems with $5 \% \mathrm{w} / \mathrm{w}$ blue dye. 
3.3. Use of PNIPAM as a stimuli responsive tool for microPADs

PNIPAM performed satisfactorily as a reagent pencil when they were shown to be made more efficient with the incorporation of a plasticizing polymer, such as PEG. Thus, it becomes possible to investigate stimuli responsive effects of PNIPAM and its incorporation into microPAD design. More specifically, it is known that the LCST of PNIPAM can be lowered with different salts following the Hoffmeister series. If the LCST can be lowered to below room temperature, high concentrations of now insoluble PNIPAM may be able to block fluid flow. Thus, PNIPAM can be potentially used as a salt responsive 'gate' that directs fluid flow in microPAD devices. In order to quantify this effect, BD was used as a visual aid to understand how the fluid front moves through microPADs both laterally and vertically.

\subsubsection{Effect of PNIPAM on vertical fluid flow}

\subsubsection{Determination of salt effects on PNIPAM LCST for simple vertical microPADs}

To determine the extent of LCST manipulation possible for PNIPAM, it was necessary to investigate the use of various salts at different concentrations. Vertical fluid flow was examined first for simple vertical flow 96 well microPADs. The devices were set up as described in Section 3.5.2.2. PNIPAM-graphite pencil was deposited onto the chromatography paper layer prior to pipetting BD and salt solutions. Figure $\mathbf{3 3}$ depicts signal intensity of $\mathrm{BD}$ and different concentrations of $\mathrm{NaCl}, \mathrm{KI}$, or $\mathrm{Na}_{2} \mathrm{SO}_{4}$. In all cases, the use of a salt and BD solution showed greater signal intensity than the control of BD 
with no salt solution. This indicates that some amount of $\mathrm{BD}$ is retained in the chromatography paper potentially as a result of manipulating the LCST of PNIPAM. Different concentrations of $\mathrm{Na}_{2} \mathrm{SO}_{4}$ and $\mathrm{NaCl}$ were investigated for the same experiment as shown in Figure $\mathbf{3 4}$ Interestingly, signal retention increased with increasing concentrations of $\mathrm{Na}_{2} \mathrm{SO}_{4}$, and the effect of signal retention was greatest at high concentrations of $\mathrm{Na}_{2} \mathrm{SO}_{4}$. For $\mathrm{NaCl}$, signal retention remained constant regardless of its concentration. Because $\mathrm{Na}_{2} \mathrm{SO}_{4}$ has greater ionic strength than $\mathrm{NaCl}$, PNIPAM is more solvated in solutions of $\mathrm{Na}_{2} \mathrm{SO}_{4}$. As a result, greater LCST manipulation is viable in solutions of $\mathrm{Na}_{2} \mathrm{SO}_{4}$, and greater amounts of insoluble PNIPAM is able to block fluid flow through the chromatography paper.

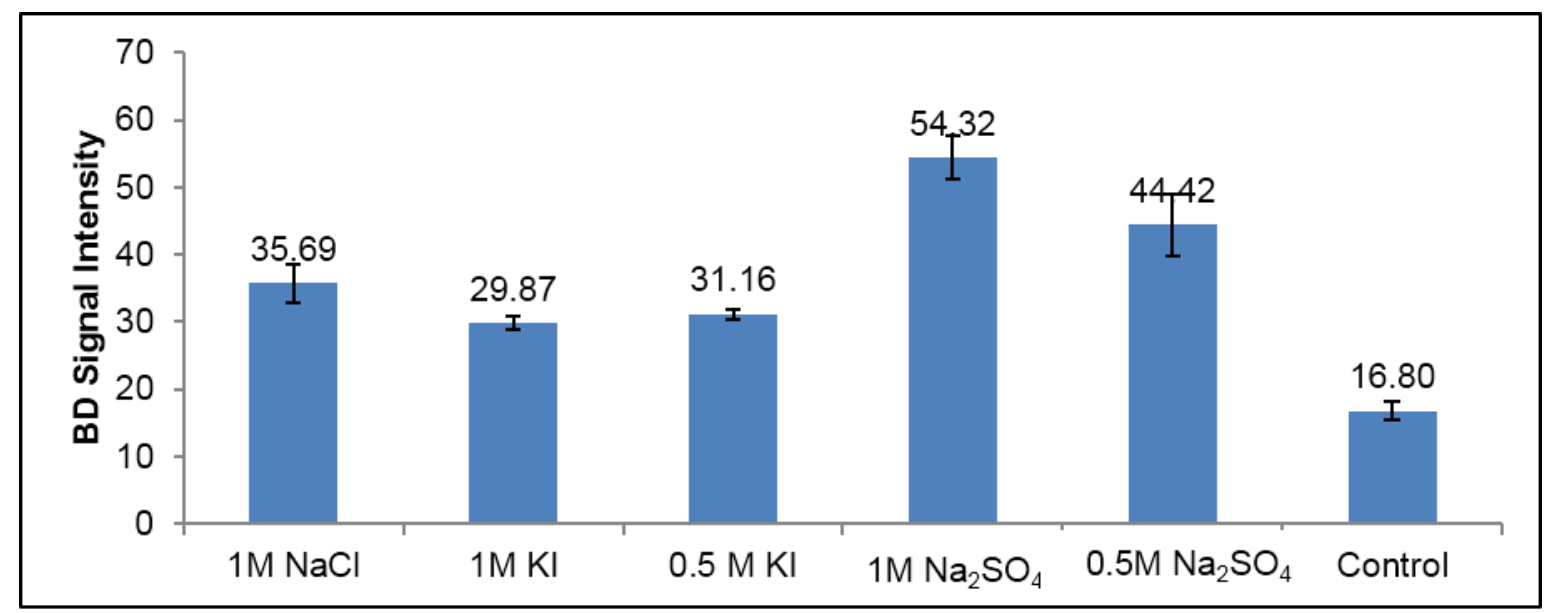

Figure 33. Effect of salt composition and concentration on $0.125 \mathrm{mM} \mathrm{BD}$ signal on a 96 well plate with pre-deposited PNIPAM-graphite. 


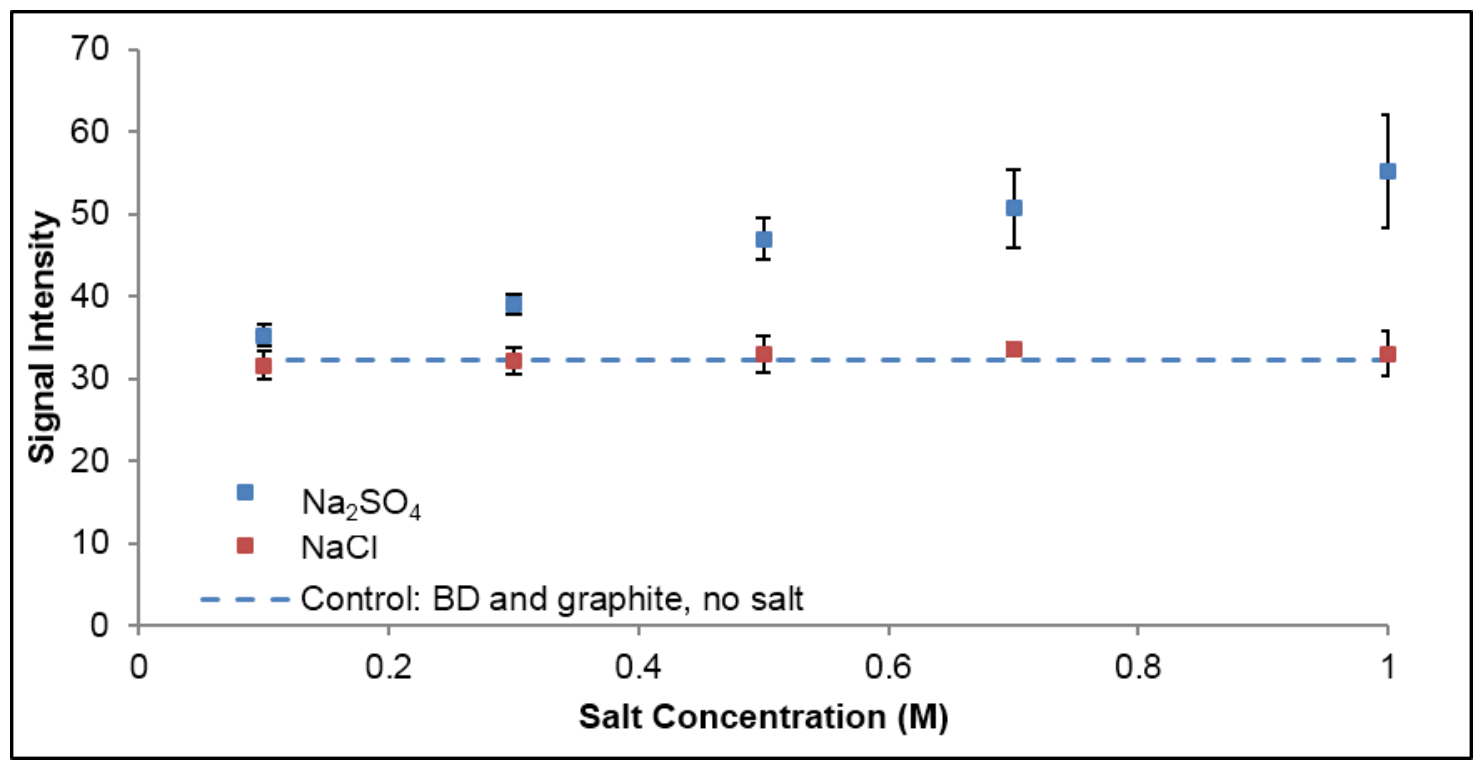

Figure 34. Effect of $\mathrm{Na} 2 \mathrm{SO} 4$ and $\mathrm{NaCl}$ concentration on $0.125 \mathrm{mM} \mathrm{BD}$ signal on a 96 well plate with pre-deposited PNIPAM-graphite.

To determine if the effects of BD signal retention were a result of PNIPAM's LCST or effects of the salt, various controls were run using the same experimental design. PNIPAM was deposited either from solution (13.51 mM or $26.79 \mathrm{mM})$ or as a reagent pencil with a $\mathrm{Na}_{2} \mathrm{SO}_{4}$ and $0.125 \mathrm{mM} \mathrm{BD}$ solution. A control was run with the salt/BD solution only and no PNIPAM. Measurements were taken at various $\mathrm{Na}_{2} \mathrm{SO}_{4}$ concentrations. Figure 35 depicts the resulting measurements of BD intensity on the chromatography paper layer. The incorporation of PNIPAM from either solution deposition or reagent pencil deposition showed no significant difference in signal retention when compared to the control with no PNIPAM. This indicates that there is some effect of BD signal retention that is a result of $\mathrm{Na}_{2} \mathrm{SO}_{4}$ concentration independent of PNIPAM. 
Alternatively, it is possible that PNIPAM is still able to affect fluid flow if it is more concentrated. In order to investigate this, it was necessary to design a more complex vertical flow microPAD (Figure 24B) where fluid flow across multiple layers of chromatography paper is possible.

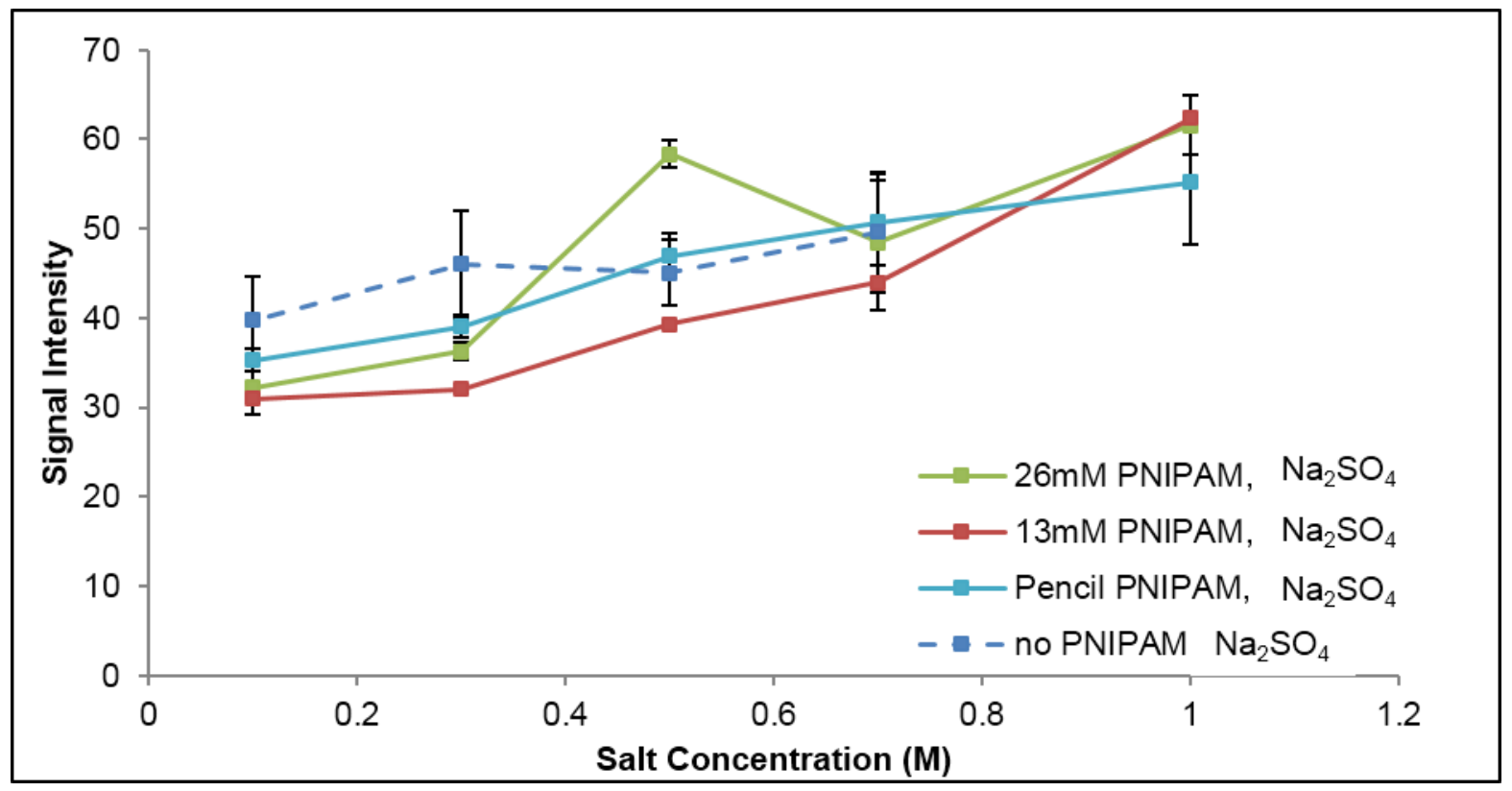

Figure 35. Effect of Na2SO4 concentration on $0.125 \mathrm{mM}$ BD signal on a 96 well plate with pre-deposited PNIPAM-graphite or PNIPAM solution. (PNIPAM solutions in DMF; all used PNIPAM-graphite DI wash control).

3.3.1.2. Manipulation of PNIPAM LCST in complex vertical flow microPADs

More complex microPADs (Figure 24B) were assembled as described in Section 3.5.2.2. To distinguish the mechanism behind salt effects and PNIPAM on vertical fluid flow, this microPAD was designed in order to understand how the fluid front moved through layers of chromatography paper. In this way, the simple quantification of BD 
signal retention in the simple vertical flow microPAD can be more fully understood. For each experiment, $0.125 \mathrm{mM} \mathrm{BD}$ was pipetted on the top Layer $\mathrm{A}$ before subsequent addition of a $1 \mathrm{M} \mathrm{Na}_{2} \mathrm{SO}_{4}$ wash. A control was also run with a $\mathrm{DI} \mathrm{H}_{2} \mathrm{O}$ wash. For experiments including PNIPAM, solutions of PNIPAM were pipetted and allowed to dry in Layer B, Layer B and C, or Layer B, C, and D at different volumes of addition prior to the microPAD assembly. Figure $\mathbf{3 6}$ shows the results of this experiment. For the control that was run with the $\mathrm{DI} \mathrm{H}_{2} \mathrm{O}$ wash, the greatest signal was found in the bottom Layer D. The control run with the $1 \mathrm{M} \mathrm{Na}_{2} \mathrm{SO}_{4}$ wash showed signal retention in the top Layer $\mathrm{A}$, and signal intensity decreased with lower chromatography paper layers. This agrees with the results from the simple vertical flow microPAD as discussed earlier. The greatest signal retention in Layer A occurred when 26.79 mM PNIPAM was incorporated in Layers B and C or Layers B, C, and D at $2 \mu \mathrm{l} /$ layer. This suggests that PNIPAM works in conjunction with the salt wash to manipulate fluid flow in a vertical microPAD. 


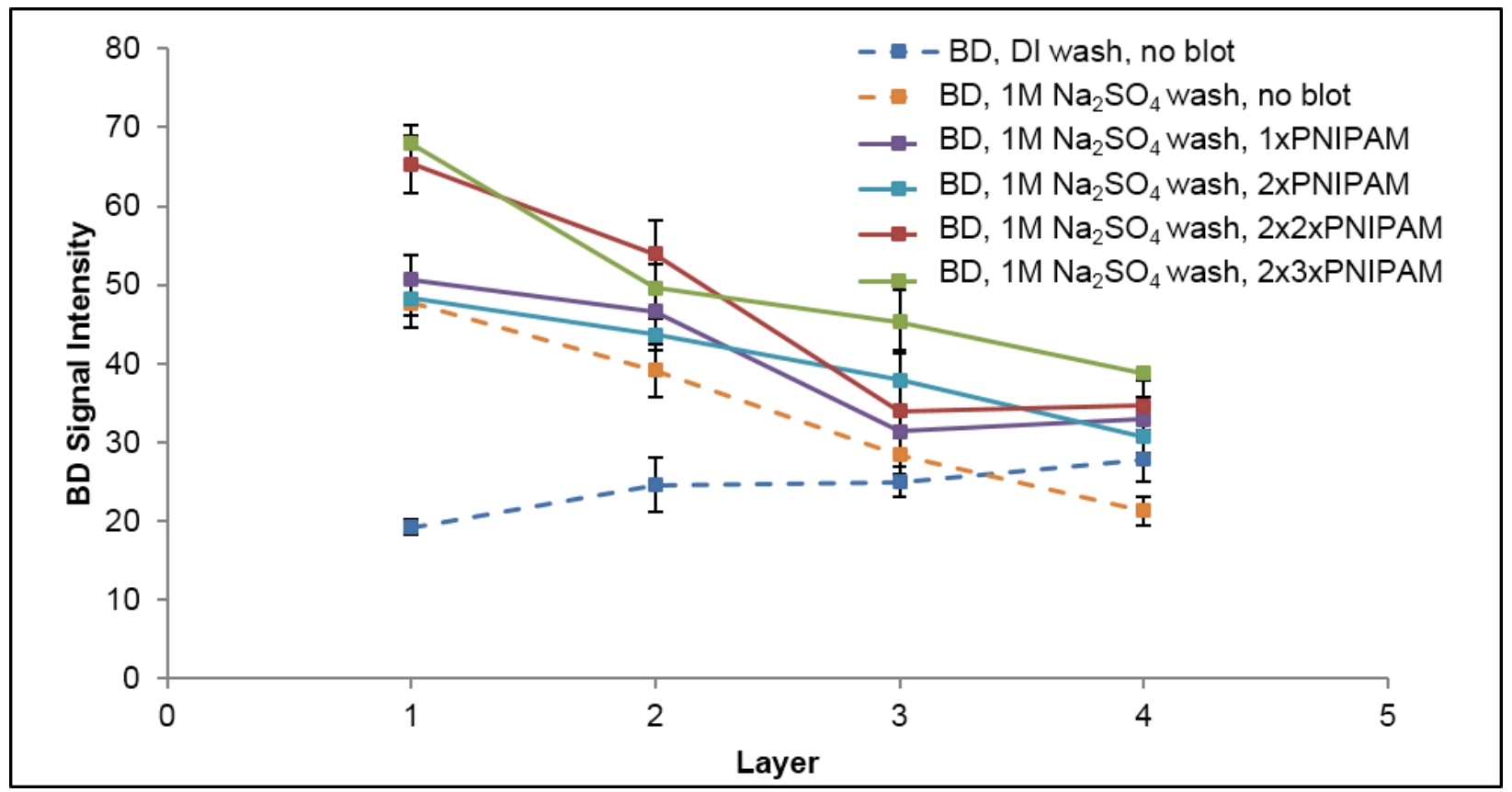

Figure 36. Complex vertical microPAD with various amount of PNIPAM doping. 3.3.1.3. Contact angle studies for complex vertical flow microPAD

In observing the previous vertical flow microPAD experiments, it was noted that the use of PNIPAM appeared to slow down the ability of solution to fully wet the addition zone of the device. Thus, contact angle measurements were taken every 10 seconds for one minute on vertical flow microPADs with PNIPAM deposited on Layer A in order to quantifiably understand this phenomenon. Further, the effect of molecular weight and concentration of PNIPAM were investigated by testing a $16 \mathrm{k}$ and $4 \mathrm{k} \mathrm{g} / \mathrm{mol}$ PNIPAM.

Figure 37 shows the crude comparison of contact angle measurements for a DI wash with no PNIPAM (Figure 37A), for a $1 \mathrm{M} \mathrm{Na}_{2} \mathrm{SO}_{4}$ wash through a microPAD with 
4k PNIPAM solution deposited in Layer A (Figure 37B), or Layers A, B, and C (Figure 37C). For a microPAD with simply $\mathrm{DI}_{2} \mathrm{O}$, the chromatography paper was fully wetted within the first 10 seconds. After one minute, the average contact angle was below $40^{\circ}$. For the samples with one or more layers of PNIPAM incorporated into the microPAD, the surface did not completely wet after one minute. In both cases, the initial contact angle was much greater $\left(>85^{\circ}\right)$ than the initial contact angle of the control run $\left(57^{\circ}\right)$. When PNIPAM was delivered to the top layer, the contact angle was maintained above $60^{\circ}$ for 20s. If PNIPAM was delivered to multiple layers of the device, (Figure 37C), the contact angle was maintained above $60^{\circ}$ for 30 s. These results indicate that PNIPAM works in conjunction with a salt wash to help impede vertical fluid flow. Further, greater amounts of PNIPAM in the microPAD appear to retard the wetting rate of the salt solution. 
A
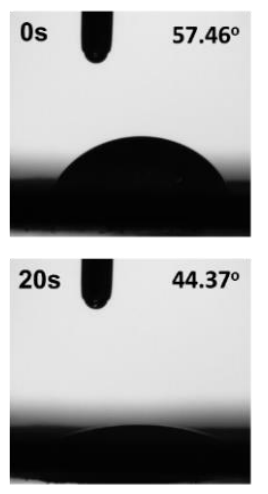

$40 \mathrm{~s}$

$40.98^{\circ}$

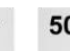

50s

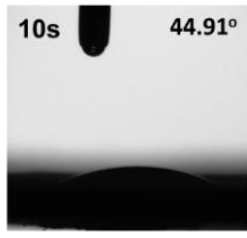

30 s

$40.96^{\circ}$

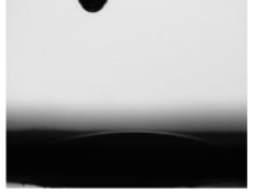

$39.72^{\circ}$

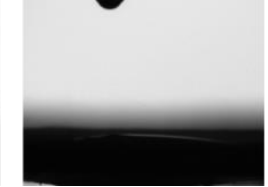

B
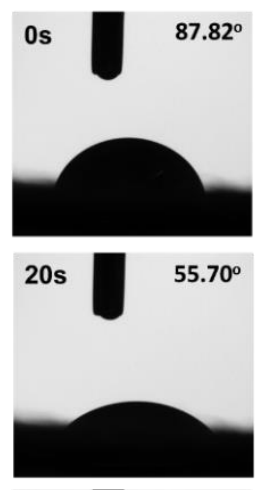

$40 \mathrm{~s}$

$51.46^{\circ}$

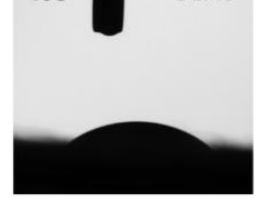

C
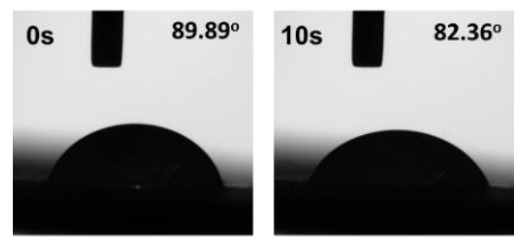

20s

$76.73^{\circ}$

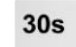

$60.55^{\circ}$

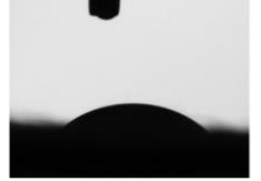

$50 \mathrm{~s}$

$49.59^{\circ}$

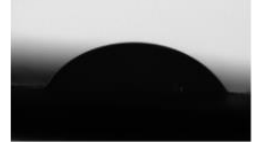

40s

$46.15^{\circ}$

50s

$42.00^{\circ}$

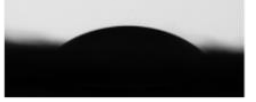

Figure 37. Contact angle measurements over time for vertical flow microPAD with 4 layers. (A) DI wash only; (B) Layer A with 26mM 4k PNIPAM and $1 \mathrm{M} \mathrm{Na}_{2} \mathrm{SO}_{4}$; (C) Layer A, B, C with 26mM 4k PNIPAM and $1 \mathrm{M} \mathrm{Na}_{2} \mathrm{SO}_{4}$.

In order to fully understand the effects of PNIPAM on vertical flow, both molecular weight dependence and concentration dependence was investigated when PNIPAM was deposited on Layer A only. For this experiment, a 4k PNIPAM and a 16k PNIPAM was used. Additionally, $6 \mathrm{mM} 16 \mathrm{k}$ PNIPAM and $26 \mathrm{mM}$ 4k PNIPAM were selected specifically to deliver the equivalent mass of PNIPAM onto Layer A. 9mM and $6 \mathrm{mM}$ 16k PNIPAM was chosen to compare the contact angle rate dependence on polymer concentration. Figure 38 depicts contact angle measurements for 9mM and 6mM 16k PNIPAM, 26mM PNIPAM with a $1 \mathrm{M} \mathrm{Na}_{2} \mathrm{SO}_{4}$ wash, and for a $\mathrm{DI} \mathrm{H}_{2} \mathrm{O}$ control wash with no PNIPAM. Figure 39A shows the corresponding measurements for 6 replicates of each sample over 60 
seconds for both $\mathrm{DI} \mathrm{H}_{2} \mathrm{O}$ and $1 \mathrm{M} \mathrm{Na}_{2} \mathrm{SO}_{4}$ washes. Figure 39B shows the same set of data for the first 30 seconds without the $\mathrm{DI} \mathrm{H}_{2} \mathrm{O}$ wash samples for clarity. Both figures show a DI wash control with no PNIPAM present. 
A. 9 mM 16k PNIPAM

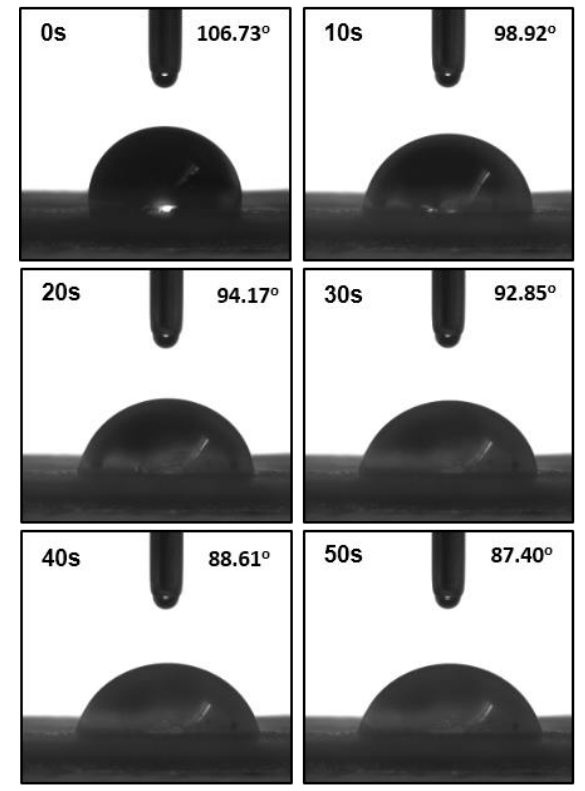

C. $26 \mathrm{mM}$ 4k PNIPAM
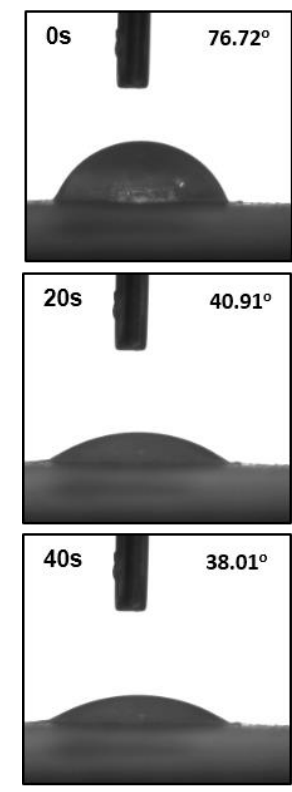
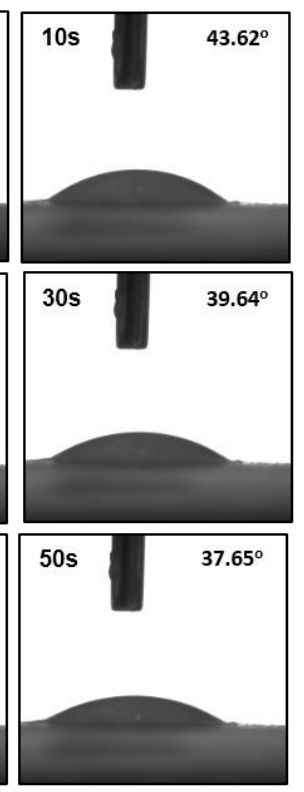

B. $6 \mathrm{mM}$ 16k PNIPAM

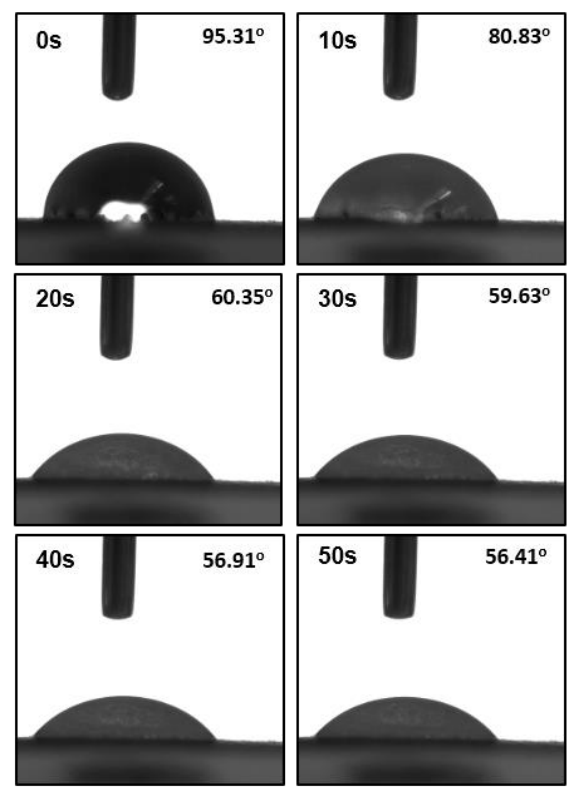

D. DI wash only, no PNIPAM

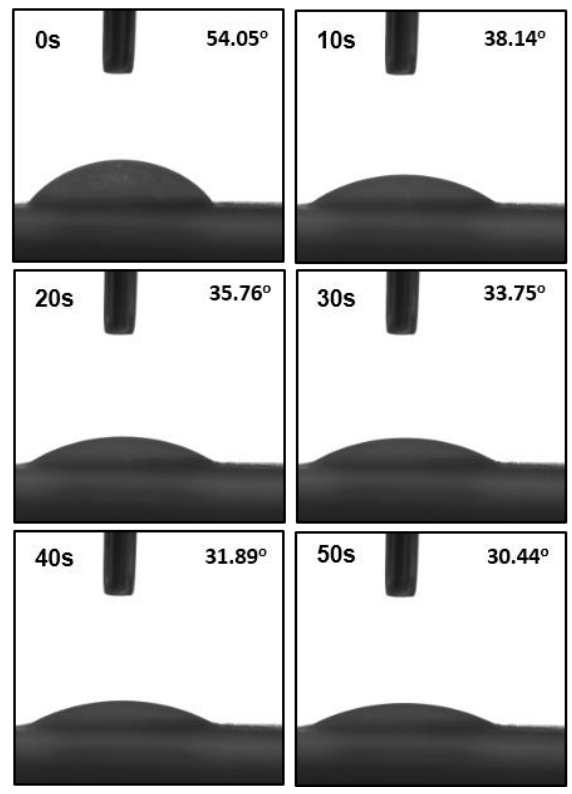

Figure 38. Contact angle measurements over a minute for (A) $9 \mathrm{mM}$ 16k PNIPAM, (B) 6 $\mathrm{mM} 16 \mathrm{k}$ PNIPAM, (C) $26 \mathrm{mM}$ 4k PNIPAM with a $1 \mathrm{M} \mathrm{Na}_{2} \mathrm{SO}_{4}$ wash, and (D) DI solution with no PNIPAM. All PNIPAM samples were deposited onto Layer A. 


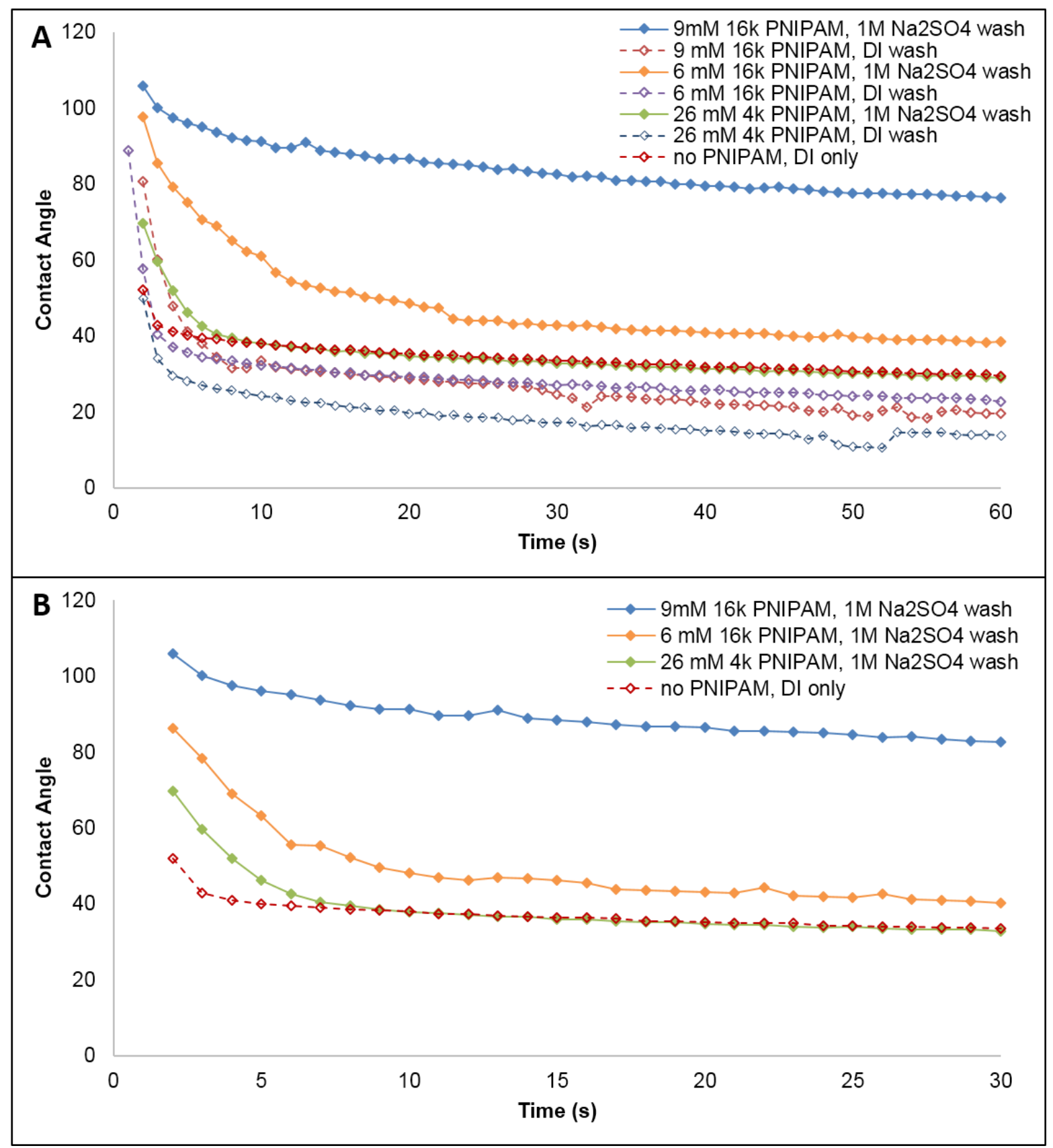

Figure 39. Contact angle vs time for varying concentrations and molecular weights of PNIPAM with either $1 \mathrm{M} \mathrm{Na}_{2} \mathrm{SO}_{4}$ or $\mathrm{DI} \mathrm{H}_{2} \mathrm{O}$ washes over (A) 60 seconds and (B) 30 seconds. 
Overall, PNIPAM samples with DI washes showed no significant statistical difference than the DI wash control with no PNIPAM. For all samples, the most significant changes in contact angle occurred in the first 30 seconds after the solution was delivered. When comparing contact angle molecular weight dependence between $26 \mathrm{mM} 4 \mathrm{k}$ PNIPAM and 6mM 16k PNIPAM, only a slight difference in their initial contact angle is observed. It is worth noting that the initial contact angle of $26 \mathrm{mM} 4 \mathrm{k}$ PNIPAM was not significantly different than the DI wash control with no PNIPAM whereas the $6 \mathrm{mM} 16 \mathrm{k}$ PNIPAM was statistically different for the first 6 seconds of measurement. This implies that there is a slight contact angle rate dependence on molecular weight. When comparing the concentration dependence with 16k PNIPAM, however, there is a significant increase in contact angle with higher concentration. At 9mM 16k PNIPAM, the contact angle remained $>75^{\circ}$ even after 60 seconds. This suggests that the retardation of contact angle rate, and the effective speed salt solutions are able to flow vertically through a microPAD device, can also be dictated by the concentration of PNIPAM.

\subsubsection{Effect of PNIPAM on lateral fluid flow}

The effect of PNIPAM on the lateral flow of fluid was investigated using a simple bone device with both solid and solution deposition. Similar to the vertical flow tests, the most efficient salt for manipulating the LCST of PNIPAM was considered first. For this experiment, $\mathrm{Na}_{2} \mathrm{SO}_{4}$ and $\mathrm{NaCl}$ were both tested at either 1 or $0.5 \mathrm{M}$ with varying concentrations of PNIPAM. Figure $\mathbf{4 0}$ summarizes the results from this test. 

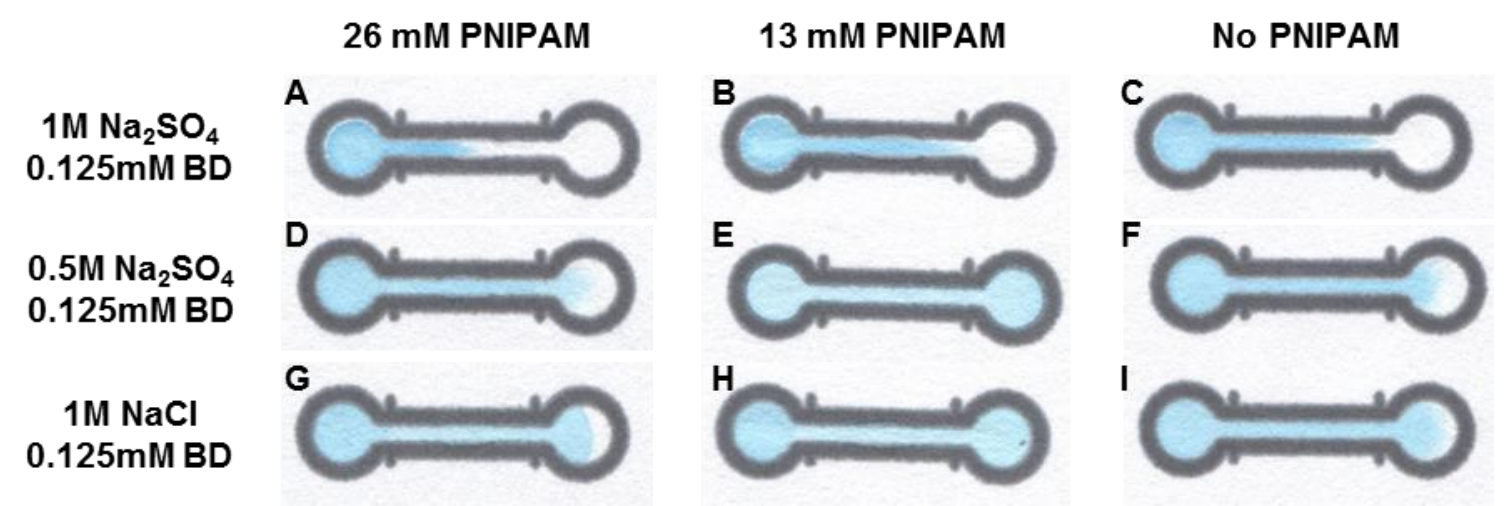

Figure 40. Salt and PNIPAM concentration variance on BD fluid flow.

The ability of PNIPAM to remain immobile on the chromatography paper was also determined by subjugating microPADs to $13 \mu$ l washes of $\mathrm{DI} \mathrm{H}_{2} \mathrm{O}$ prior to pipetting the $\mathrm{BD}$ and $\mathrm{Na}_{2} \mathrm{SO}_{4}$ solution. Figure 41 illustrates the results of this initial investigation. When only BD was delivered to the sample zone, the microPAD was consistently able to go to completion (Figure 41A and D). If the solution delivered included $1 \mathrm{M} \mathrm{Na}_{2} \mathrm{SO}_{4}$, the device would not reach completion and the blue dye would reach about halfway into the channel zone (Figure 41B and E). Finally, if the microPAD was treated to washes of $\mathrm{DI} \mathrm{H}_{2} \mathrm{O}$ with subsequent addition of $\mathrm{BD}$ and $1 \mathrm{M} \mathrm{Na}_{2} \mathrm{SO}_{4}$, the microPAD was able to reach completion (Figure 41C and F). These results indicate that PNIPAM is able to successfully block fluid flow laterally across microPADs, but fails to do so if PNIPAM is washed out with a DI $\mathrm{H}_{2} \mathrm{O}$ solution. For this initial test, it took 4 washes of $13 \mu \mathrm{I} \mathrm{DI} \mathrm{H}_{2} \mathrm{O}$ prior to the addition of blue die and salt for the PNIPAM to be ineffective. To understand the washing out effect of 
PNIPAM, Figure 42 illustrates the effects of multiple washes on PNIPAM's fluid flow efficacy for both solid and solution deposition.
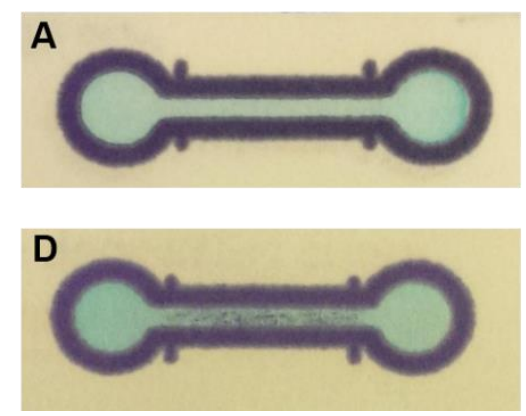
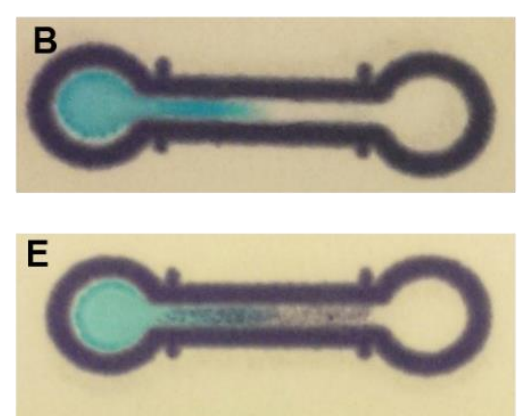
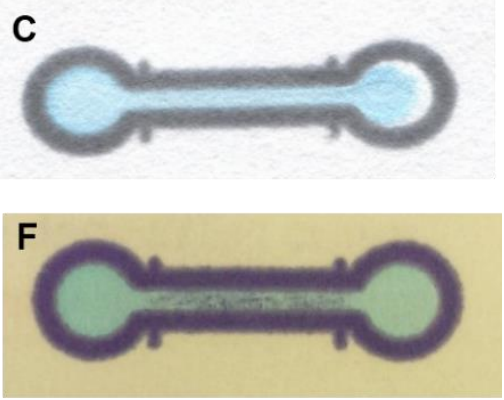

Figure 41. Solution and solid deposition of PNIPAM on lateral flow devices with $0.125 \mathrm{mM} \mathrm{BD}$ only $(A$ and $\mathrm{D})$, with $1 \mathrm{M} \mathrm{Na}_{2} \mathrm{SO}_{4}$ and $0.125 \mathrm{mM} \mathrm{BD}(\mathrm{B}$ and $\mathrm{E})$, and with $4 \times 13 \mu \mathrm{l} \mathrm{DI} \mathrm{H}_{2} \mathrm{O}$ wash prior to $1 \mathrm{M} \mathrm{Na}_{2} \mathrm{SO}_{4}$ and $0.125 \mathrm{mM} \mathrm{BD}$ addition ( $\mathrm{C}$ and $\mathrm{F}$ ).

\section{A. Solid Deposition \\ B. Solution Deposition}

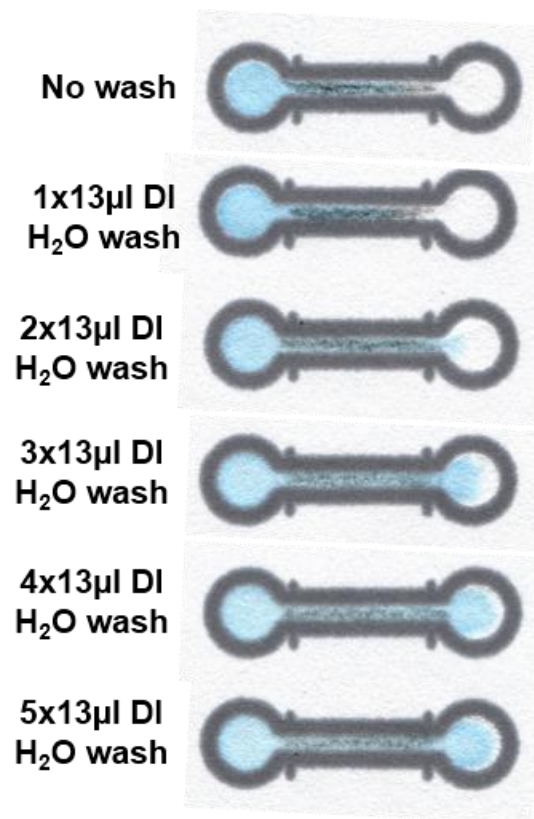

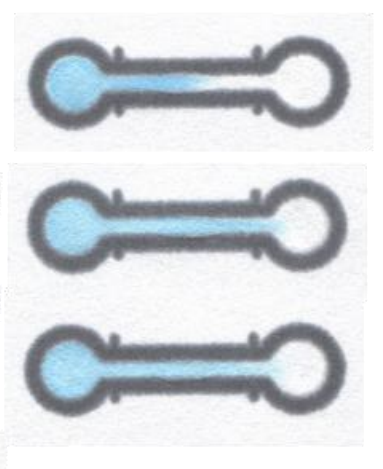

Figure 42. PNIPAM (A) solid and (B) solution deposition of 4k PNIPAM wash tolerance up to 5 times the device volume. After washing, $13 \mu \mathrm{l}$ of $1 \mathrm{M} \mathrm{Na}_{2} \mathrm{SO}_{4}$ and $0.125 \mathrm{mM} \mathrm{BD}$ was pipetted into the sample addition zone. 
In general, increasing the number of washes causes a decline in PNIPAM's ability to block fluid flow. With no washes, the blue dye stops about halfway through the device. After two washes, the blue dye is able to reach the end zone of the device. With greater than three washes, the blue dye is able to completely fill the device. This implies that PNIPAM becomes soluble and is mobile on the device after deposition. If PNIPAM can be immobilized on chromatography paper via click chemistry, it is possible for this effect to be negated.

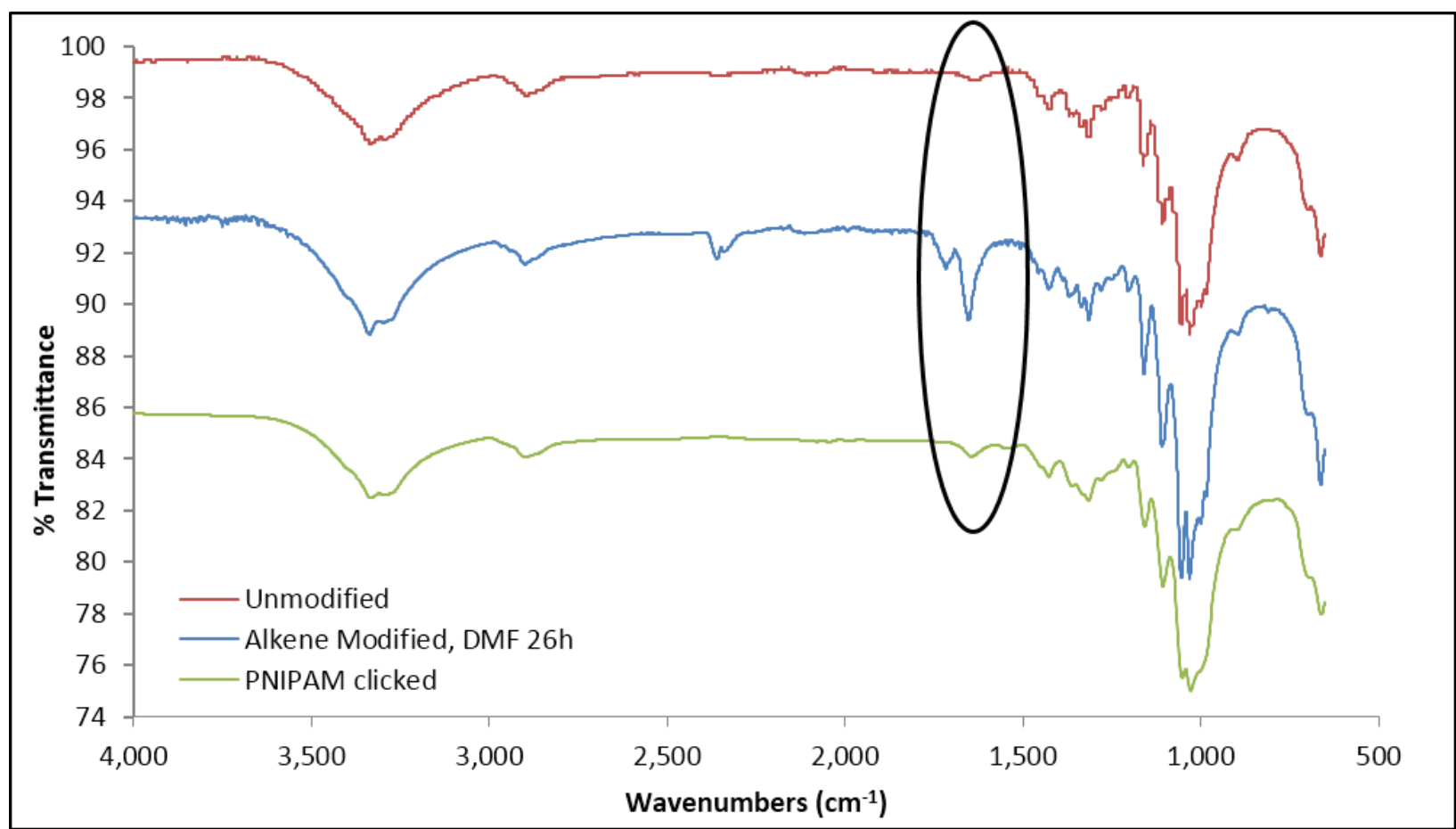

Figure 43. IR spectroscopy of unmodified, alkene functionalized, and PNIPAM functionalized chromatography paper.

The modification of chromatography paper was necessary to create a chemical handle for click chemistry of microPADs to proceed. Acryloyl chloride $(19.72 \mathrm{mmol})$ was 
allowed to stir in $100 \mathrm{~mL}$ DMF with unmodified chromatography paper for 24 hours to produce an alkene functionalized paper substrate. Prepared solutions of thiol terminated PNIPAM were then clicked onto the alkene modified paper via 20 seconds of exposure to $365 \mathrm{~nm}$ UV light in the presence of a 0.10M 2,2-dimethoxy-2-phenylacetophenone (DMPA) solution. IR analysis of unmodified, alkene functionalized, and PNIPAM clicked chromatography paper is shown in Figure 43. The presence of peaks at 1652.99 and $1716.69 \mathrm{~cm}^{-1}$ indicate the alkene functionalization of chromatography paper was successful. The subsequent disappearance of the equivalent peaks suggests that clicking PNIPAM onto the microPAD was also successful. 


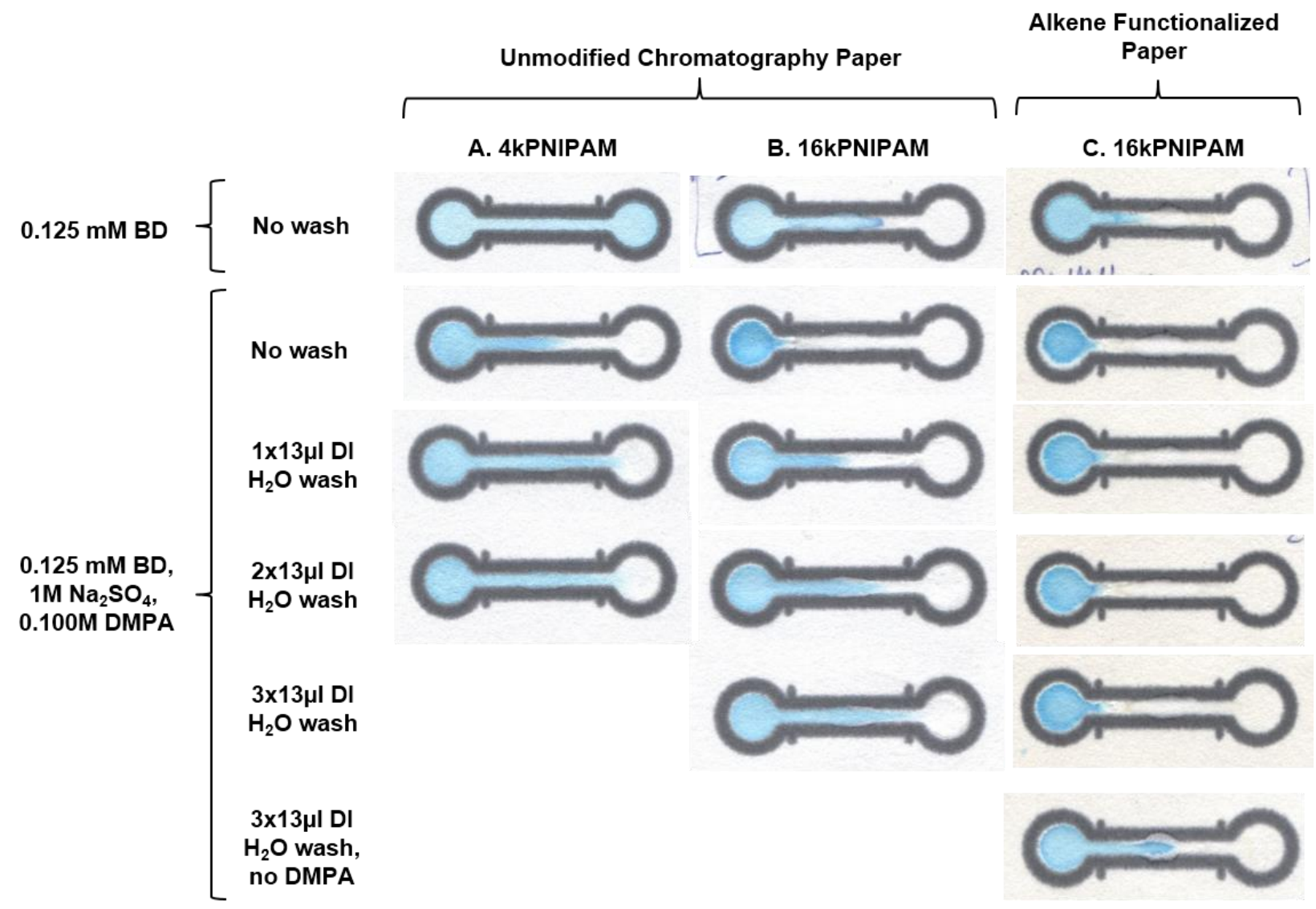

Figure 44. Comparison of $(A) 4 k$ and $(B, C)$ 16k PNIPAM efficiency when clicked onto alkene functionalized paper and not clicked on unmodified paper.

Figure $\mathbf{4 4}$ shows a comparison of lateral flow tests with the solution deposition of 4000 and $16000 \mathrm{~g} / \mathrm{mol}$ PNIPAM. 16k PNIPAM was also tested for its properties when clicked onto alkene functionalized chromatography paper. In general, even without the presence of $1 \mathrm{M} \mathrm{Na}_{2} \mathrm{SO}_{4}$, fluid flow efficiency seems to be dictated slightly by molecular weight. The lateral flow device with 4k PNIPAM was able to go to completion, whereas the device with 16k PNIPAM was not. Interestingly, when 16k PNIPAM was clicked onto 
modified chromatography paper, the BD was only able to reach about halfway through the channel zone. This implies that without click modification, some PNIPAM is still solubilized with a single BD wash. Immobilizing PNIPAM prevents the polymer from moving with the solvent front, and improves the ability of PNIPAM to block fluid flow. In a similar manner, when polymer was not clicked onto unmodified chromatography paper, DI $\mathrm{H}_{2} \mathrm{O}$ washes prior to the addition of $\mathrm{BD} / 1 \mathrm{M} \mathrm{Na} \mathrm{SO}_{4}$ solubilized PNIPAM regardless of its molecular weight. The higher molecular weight PNIPAM was more tolerant to $\mathrm{DI}_{2} \mathrm{O}$ washes compared to the 4k PNIPAM. When the 16k PNIPAM was clicked onto modified paper, however, washes had no effect on the ability of PNIPAM to block fluid flow. Even with 3 device volume washes, BD was not able to leave the sample addition zone of the device. Without the presence of the photoinitiator, DMPA, PNIPAM is unable to be covalently bound to the paper, and the performance of this device is akin to unclicked PNIPAM. These results imply that clicking PNIPAM onto the chromatography paper was successful, and the fluid flow blocking properties of PNIPAM is reinforced when it is immobilized onto alkene functionalized paper.

\subsection{Reagent Condition tolerance in preparation for photoinitiated click chemistry}

The successful employment of click chemistry for PNIPAM onto microPADs spurred the development of a novel reagent type to click onto the paper substrate. In particular, any enzyme with free cysteine residues may be able to be immobilized on chromatography paper via click chemistry given that the active site is not compromised. 
Effectively, this would become an inexpensive alternative to other complex protein immobilization techniques, which often require surface blocking to prevent unwanted nonspecific protein binding. For this work, an initial investigation on the condition tolerance of HRP to thiolene click chemistry conditions was conducted in preparation for covalently binding the enzyme to a paper substrate.

\subsubsection{Solvent and UV irradiation tolerance of HRP}

To develop a novel microPAD device for the photo-based immobilization of HRP, it was necessary to understand how different solvent and irradiation conditions affect the activity of HRP. Serial dilutions of $20,10,5,3,1,0.5$, and $0.1 \mathrm{U} / \mathrm{mL}$ HRP were exposed to UV light $(365 \mathrm{~nm})$ between $0-60$ seconds on a 96 well microPAD. The results for this test are shown in Figure 45A. As UV exposure time increases, the activity of HRP is diminished. In other words, the generation of radical oxidative species in the system from UV irradiation diminishes the activity of HRP rapidly.

The effect of DMPA in DMF on the activity of HRP was also investigated. Specifically, the importance of HRP/DMPA order of addition was investigated. In one case, HRP dilutions were first added to the microPAD and allowed to dry for five minutes before subsequent addition of a 0.017 M DMPA solution in DMF. For a different sample, DMPA solution was pipetted onto the microPAD and allowed to dry prior to the addition of HRP dilutions. The results of this test are shown in Figure 45B. When HRP is added first, the 
subsequent addition of DMPA solution denatures the enzyme and results in no activity at any concentration of HRP. When the DMPA solution is allowed to dry first before the addition of HRP, however, the activity of HRP is comparable to a control experiment with no DMPA/DMF addition. Thus, the presence of an organic solvent clearly denatures HRP and eliminates its activity. When the solvent is allowed to evaporate, however, HRP performs as normal even in the presence of DMPA.

Figure $45 \mathrm{C}$ depicts the activity of HRP dilutions with both the addition of $0.017 \mathrm{M}$ DMPA in DMF and exposure to UV irradiation at 10 and 30 seconds. DMPA solution was added prior to the addition of enzyme for this experiment. Again, as UV exposure time increases, the activity of the enzyme diminishes. Because the results of these plots are analogous to the results of the UV irradiation experiment, it can be assumed that HRP will lose activity after $\geq 10$ seconds of UV exposure, given that the DMPA/DMF solution is added and allowed to dry prior to the addition of HRP. In preparation for thiolene click chemistry, it seems that these conditions are optimal to covalently bind HRP to the paper substrate. Further work still needs to be done to test the effectiveness of this enzyme immobilization technique. In the future, it may also be interesting to see the effects of UV irradiation on enzyme stability in the presence of PEG as well. Because previous work in this work has shown the improvement of HRP activity in the presence of PEG, even at low enzyme concentrations, it is possible that PEG can minimize enzyme denaturation from UV irradiation as well. 


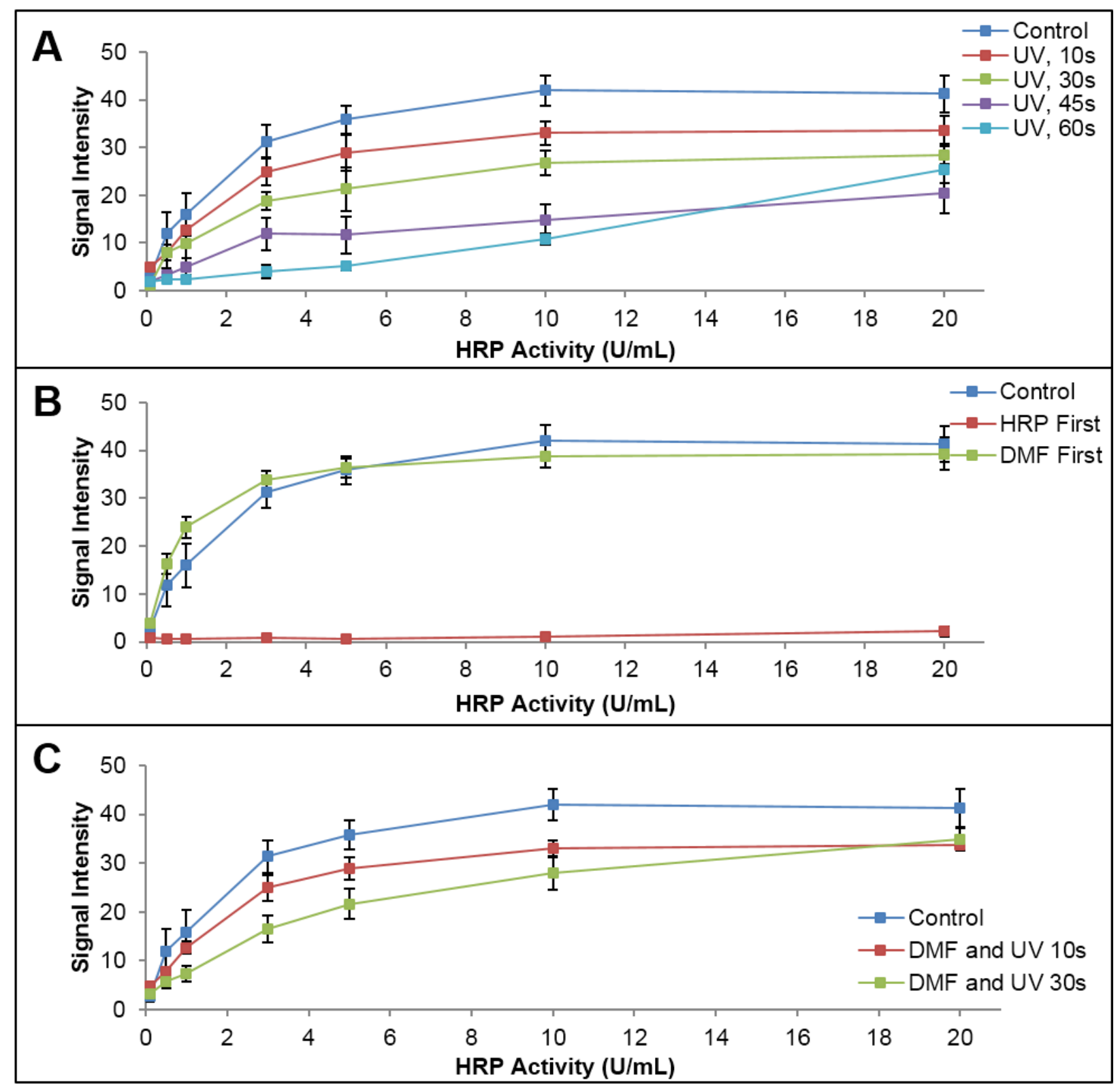

Figure 45. Comparison of HRP condition tolerance to (A) UV exposure time, (B) solvent/reagent order of addition , and (C) DMF and UV tolerance at varying concentrations of $\operatorname{HRP}(20,10,5,3,1,0.5$, and $0.1 \mathrm{U} / \mathrm{mL} \mathrm{HRP})$. 


\subsubsection{Reagent Wash Tolerance}

\subsubsection{Reagent Lateral Flow Wash Tolerance}

In order to determine the need to covalently bind reagents onto microPADs, the wash tolerances of both $\mathrm{HRP}$ and $\mathrm{BD}$ were determined as representative reagents. A simple, 'caterpillar' device was employed for determining reagent tolerance to several washes. Each sample was solution deposited into Zone 2, and a $\mathrm{DI} \mathrm{H}_{2} \mathrm{O}$ wash solution was pipetted into Zone 1. It is possible to determine the flow of a reagent across the device by measuring the colorimetric reagent signal intensity at each sample zone. Figure 46 shows the lateral flow device used, as well as the corresponding signal responses for both HRP and BD experiments.

Interestingly, for HRP, the greatest signal intensity at all concentrations tested was found in Zone 2. This indicates that HRP may already interact with the chromatography paper substrate such that most of the signal remains in the initial sample zone. Although some of the enzyme is distributed into Zones 3 and 4 , about $80 \%$ of the maximum signal possible, as compared to a $20 \mathrm{U} / \mathrm{mL} \mathrm{HRP}$ signal deposited on a 96 well microPAD, remained in Zone 2. In the case of HRP, this response profile does not necessitate the use of click chemistry to covalently bind to the paper substrate. Regardless of whether or not HRP has free cysteine residues would be available for click chemistry, strong interactions between this specific enzyme and the paper already limit the utility of pursuing covalent modification. 
When BD was washed through the device using the same methodology, the greatest signal intensity was found to be in Zone 7. Because BD is inherently water soluble, it is appropriate to expect that much of the signal response would be washed away and found in the end zone. In the future, this would be the appropriate response profile of a reagent that would need to be covalently bound to the surface of the microPAD via thiolene click chemistry. Although the scope of this project investigated HRP extensively, an alternative enzyme that shows a wash response profile similar to BD would be more appropriate to investigate in the future. It is important to note, however, that the response profiles of an alkene modified paper substrate may be different by the nature of their surface functionality. In those cases, more response profiles must be investigated appropriately to determine whether covalent modification is necessary. 


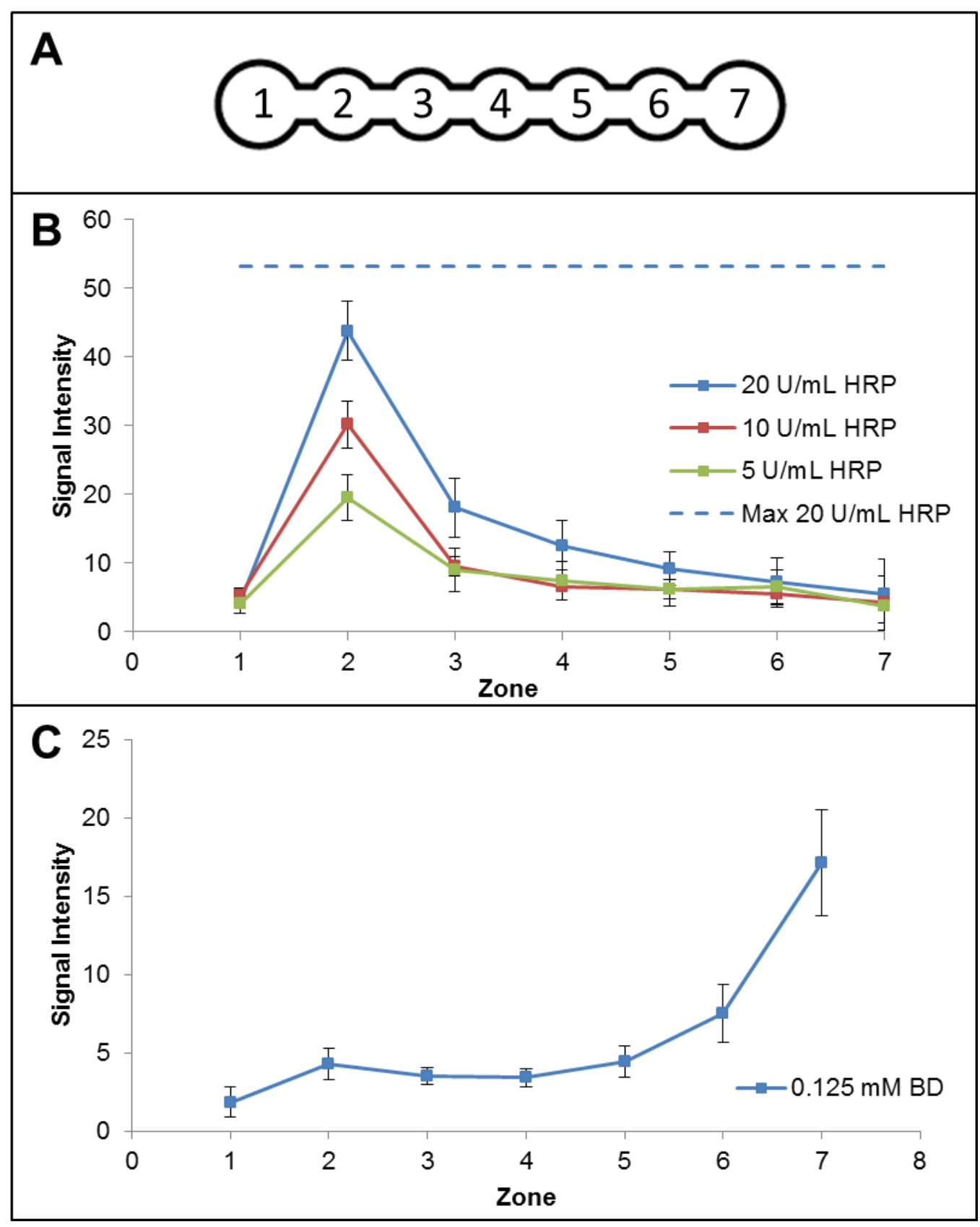

Figure 46. (A) Caterpillar lateral flow device used to determine reagent wash tolerance. The response profiles of (B) HRP and (C) $0.125 \mathrm{mM} \mathrm{BD}$ are shown. 


\subsubsection{Reagent Vertical Flow Wash Tolerance}

As with the lateral flow tests discussed, the wash tolerance of reagents must be determined in order to understand the necessity of covalently binding reagents onto the paper substrate. To determine the vertical wash tolerance of reagents, a complex vertical stack microPAD was used, as shown in Figure 24. To determine the wash tolerance, reagent sample and a subsequent wash solution was deposited onto the top layer, Layer 1 , of a folded microPAD. The colorimetric response profiles of each layer could be determined after unfolding and scanning the device. The response profile for both 0.125 $\mathrm{mM} \mathrm{BD}$ and $20 \mathrm{U} / \mathrm{mL} \mathrm{HRP}$, respectively, is shown in Figure 47A and Figure 47B.

Similar to the lateral flow device, the BD sample showed the highest signal response in the bottom layer, Layer 4 . This is the ideal response profile of a water soluble reagent that would necessitate covalent modification if desired. In the case of $\mathrm{HRP}$, the highest signal was found to be in the top layer, Layer 1. Interestingly, the number of washes through the device affected the signal response at each layer. When more than one wash was introduced, the signal at layers 2,3 , and 4 , were found to decrease significantly. Most likely, the drying process of multiple washes denatures HRP. Inherently, utilizing multiple washes also attempts to push the enzyme forcibly through each substrate layer. Presumably, the additive effects of both drying out and pressure from wash solutions denature HRP and reduce the signal response at the intermediate and bottom layer of the microPAD. In general, this response profile reflects a reagent that 
would not require covalent modification to the substrate. It is clear from both the lateral and vertical flow tests that HRP interacts with the paper substrate sufficiently to withstand wash solutions through the device. In the future, an enzyme with a wash response more similar to BD would necessitate the investigation of covalent modification.

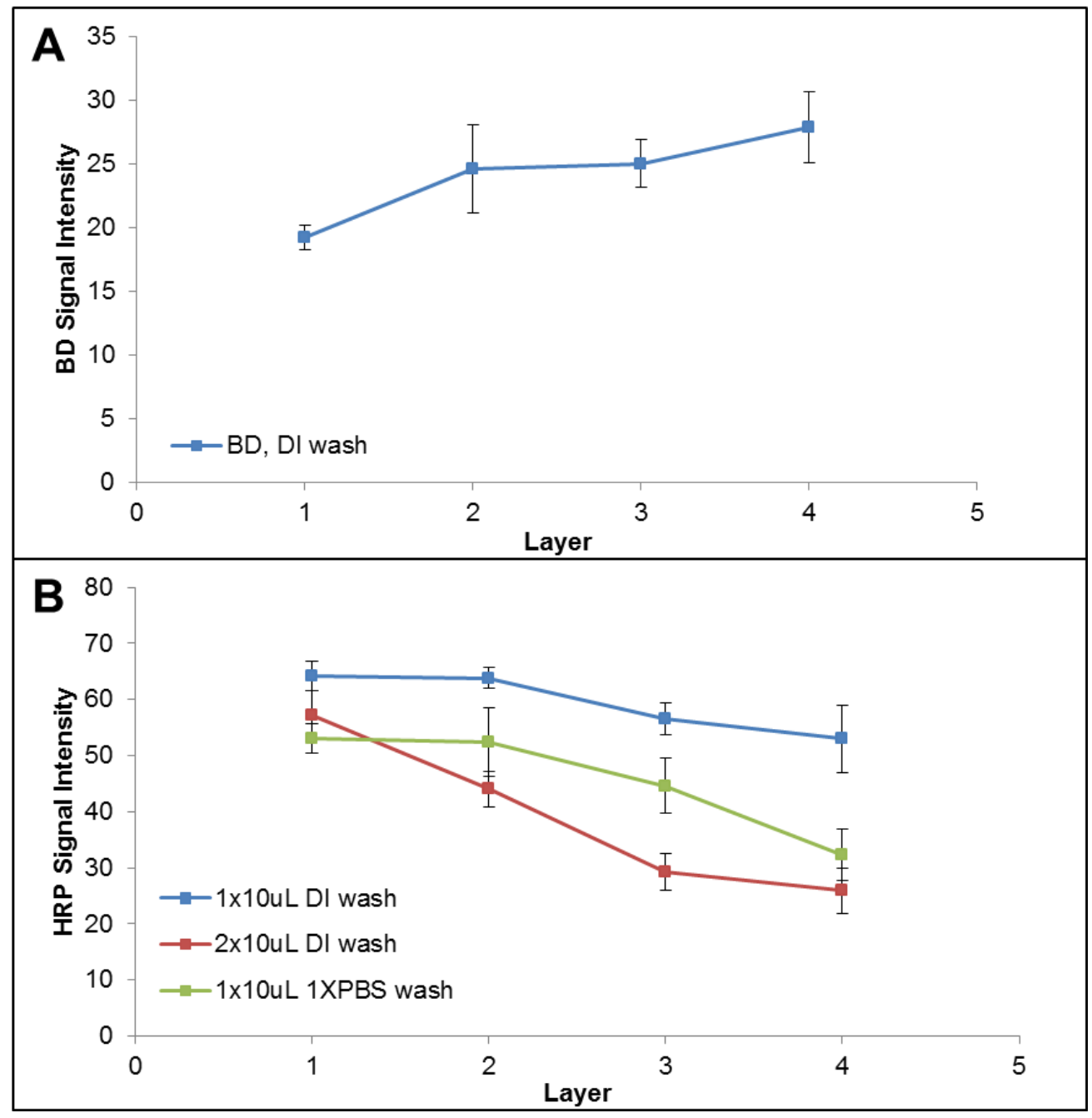

Figure 47. Vertical flow wash tolerance of (A) $0.125 \mathrm{mM} \mathrm{BD}$ and (B) $20 \mathrm{U} / \mathrm{mL} \mathrm{HRP}$. 
3.5. Experimental Methods and Materials.

\subsubsection{Materials}

All reagents were purchased from commercial sources unless otherwise stated. The following chemicals were used: N-isopropylacrylamide (NIPAM, Sigma Aldrich), azobisisobutyronitrile (AIBN, Sigma Aldrich), propylamine (Sigma Aldrich), acryloyl chloride (Sigma Aldrich). All GPC samples utilized an Agilent 1200 refractive index detector and were performed in THF at $30^{\circ} \mathrm{C}$ with a flow rate of $1.500 \mathrm{~mL} \mathrm{~min}{ }^{-1}$.

3.5.2. PNIPAM Synthesis of dithiobenzene and nitrile terminated PNIPAM

\subsubsection{Synthesis of carboxylic acid terminated PNIPAM}

The synthesis of carboxylic acid terminated PNIPAM was conducted by Anthony Varni. PNIPAM was synthesized via RAFT polymerization. In a $50 \mathrm{~mL}$ round bottom flask, $\operatorname{NIPAM}(7.89 \mathrm{~g}, 69 \mathrm{mmol})$ and RAFT agent $(0.3985 \mathrm{~g}, 1.57 \mathrm{mmol})$ were dissolved in THF. AIBN $(0.248 \mathrm{~g}, 1.5 \mathrm{mmol})$ was added to the reaction flask which was sealed and purged with $\mathrm{N}_{2}$ gas. The reaction was placed in an oil bath at $60^{\circ} \mathrm{C}$ to yield the carboxylic acid terminated PNIPAM. The scheme for this synthesis is shown in the first step of Scheme 1.

3.5.2.2. Synthesis of thiol terminated PNIPAM

The synthesis of thiol terminated PNIPAM was conducted by Karoline Eckhart. PNIPAM was synthesized via RAFT polymerization. In a round bottom flask, NIPAM 
(19.3133 g, $0.17 \mathrm{~mol})$ and RAFT agent $(0.2569 \mathrm{~g}, 1.01 \mathrm{mmol})$ were dissolved in THF. AIBN (0.0858 g, $0.5 \mathrm{mmol})$ was then added and stirred. The reaction flask was capped and purged under $\mathrm{N}_{2}$ for 50 minutes. The reaction flask was then placed in an oil bath at $60^{\circ} \mathrm{C}$ for 6 hours to yield a carboxylic acid terminated PNIPAM. Purged propylamine $(0.4$ $\mathrm{mL}, 4$ eq) was added to the reaction flask and allowed to stir overnight. The polymer was precipitated in hexane and analyzed via GPC. The scheme for this synthesis is shown in

\section{Scheme 1.}

\subsubsection{Manipulation of fluid flow via PNIPAM LCST alteration}

\subsubsection{Lateral fluid flow manipulation}

Both simple and complex lateral flow devices, as depicted in Figure 23, were used to understand the manipulation of lateral fluid flow on microPADs.

For a simple bone device, both solution deposition and reagent pencil deposition of PNIPAM was tested in the channel zone. On a suspended bone device, a $1.5 \mu \mathrm{l}$ of either $13.51 \mathrm{mM}$ or $26.79 \mathrm{mM}$ PNIPAM in DMF was deposited into the channel and allowed to dry for 10 minutes. Alternatively, a PNIPAM-graphite reagent pencil was used to evenly deposit the polymer into the channel zone. Then, $13 \mu \mathrm{l}$ of a salt and $0.125 \mathrm{mM}$ BD solution were pipetted onto the sample addition zone of the bone device. MicroPADs were allowed to dry for 30 minutes and each corresponding end zone was subsequently scanned and analyzed in ImageJ. Both $\mathrm{NaSO}_{4}$ and $\mathrm{NaCl}$ were tested at 1 and $0.5 \mathrm{M}$ in 
ten replicates for solution deposition based experiments. $\mathrm{NaSO}_{4}$ and $\mathrm{NaCl}$ were tested at $1 \mathrm{M}$ in ten replicates for reagent pencil based experiments.

For complex, 'caterpillar', lateral flow devices (Figure 23B), the microPAD was suspended tautly and $1 \mu \mathrm{l}$ of $0.125 \mathrm{mM} \mathrm{BD}$ was pipetted into zone 2 . Then $33 \mu \mathrm{l}$ of $\mathrm{DI}$ $\mathrm{H}_{2} \mathrm{O}$ was pipetted into zone 1 . The device was allowed to dry for 30 minutes and scanned and analyzed in ImageJ.

\subsection{PNIPAM lateral flow wash tolerance}

For a simple bone device, wash tolerance for the solution deposition of PNIPAM was tested. On a suspended bone device, a $1.5 \mu \mathrm{l}$ of $26.79 \mathrm{mM}$ PNIPAM in DMF was deposited into the channel and allowed to dry for 10 minutes. $13 \mu \mathrm{L}$ washes of deionized $\mathrm{H}_{2} \mathrm{O}$ were pipetted into the sample addition zone of the device. Then, $13 \mu \mathrm{l}$ of $1 \mathrm{M} \mathrm{Na}_{2} \mathrm{SO}_{4}$ and $0.125 \mathrm{mM} \mathrm{BD}$ solution were pipetted onto the sample addition zone of the bone device. MicroPADs were allowed to dry for 30 minutes and each corresponding end zone was subsequently scanned and analyzed in ImageJ. PNIPAM wash tolerance was tested with 1, 2, and 3 consecutive washes. This test was run in 10 replicates.

\subsubsection{Vertical fluid flow manipulation}

Both simple and complex vertical flow devices, as depicted in Figure 24, were used to understand the manipulation of vertical fluid flow on microPADs. 
For a suspended, simple 96 well microPAD, $1 \mu$ of PNIPAM in DMF was deposited into each well and allowed to dry for 10 minutes. Alternatively, a PNIPAM-graphite reagent pencil was used to evenly deposit polymer onto each well. Blotting paper was then placed underneath the chromatography paper layer to promote fluid flow through the top layer. Then, a $10 \mu \mathrm{l}$ wash of salt and $0.125 \mathrm{mM} \mathrm{BD}$ solution was pipetted onto each zone. A spatula tip was used to press the area surrounding each well to ensure full contact between the chromatography paper layer and the blotting paper layer. The microPAD was then resuspended and allowed to dry for 30 minutes before scanning and analysis in ImageJ. This test was conducted in 10 replicates. Salt solutions of $1,0.7,0.5,0.3$, and $0.1 \mathrm{M}$ of $\mathrm{Na}_{2} \mathrm{SO}_{4}$ and $\mathrm{NaCl}$ were tested. $1 \mathrm{M} \mathrm{KI}$ was also tested. Both 13.51 and $26.79 \mathrm{mM}$ PNIPAM solutions were tested for these experiments.

For a complex, stacked vertical flow device as depicted in Figure 24B, each microPAD was folded along perforations to align each layer design exactly. A hole was punched in the center of the device to allow for a screw to secure two plastic covers on the top and bottom of the device and ensure even contact throughout the device. Each plastic cover was laser cut with an opening at each deposition zone. From top to bottom, layers were labeled in alphabetical order, utilizing four layers total. Prior to folding, a solution of 26.79 mM PNIPAM was pipetted into Layer B or Layer B and Layer C and allowed to dry for 10 minutes. The PNIPAM solution was pipetted at either $1 \mu \mathrm{L}$ or $2 \mu \mathrm{L}$ volumes. Each microPAD was then assembled as described. $1 \mu \mathrm{L}$ of $0.125 \mathrm{mM}$ BD and 
then $10 \mu \mathrm{L}$ of a $1 \mathrm{M} \mathrm{Na} \mathrm{SO}_{4}$ was pipetted on top of Layer A. The microPAD was allowed to dry for 30 minutes before unassembling the device to scan and analyze in ImageJ.

\subsubsection{Contact angle studies on complex vertical flow microPADs}

A goniometer was set up to measure the contact angles for the experiment described in 2.8.2 with the complex, stacked vertical flow microPADs. $1 \mu \mathrm{L}$ of PNIPAM was pipetted onto Layer 1 and allowed to dry for 10 minutes prior to folding each device. To minimize obstruction from the camera's field of vision, pairs of strong magnets were placed at the side of each deposition zone to maintain even pressure and contact between each layer. Because the nature of the experiment did not require analysis of the colorimetric response, sample runs for this test were not dried and scanned. 8 replicate measurements were taken every 10 seconds for one minute after the deposition of $10 \mu \mathrm{L}$ of $1 \mathrm{M} \mathrm{Na}_{2} \mathrm{SO}_{4}$.

3.5.4. Fabrication of alkene-functionalized chromatography paper.

A full sheet of chromatography paper $(3.4102 \mathrm{~g}, \sim 21.03 \mathrm{mmol}$ anhydroglucose units), Acryloyl chloride $(0.147 \mathrm{~mol})$, and DMF $(150 \mathrm{~mL})$ were stirred in a covered Pyrex rectangular glass baking dish for 7 hours. After washing, the paper was allowed to dry for 2 hours and was characterized via FT-IR spectroscopy. Different microPAD designs were wax printed onto each sheet as necessary and baked at $145^{\circ} \mathrm{C}$ for 15 minutes prior to testing. 


\subsubsection{Condition tolerance of HRP}

\subsubsection{DMPA/DMF tolerance of HRP via solution deposition}

The tolerance of serially diluted HRP solutions was tested on a paper based 96 well plate. 20, 10, 5, 3, 1, 0.5, and $0.1 \mathrm{U} / \mathrm{ml} \mathrm{HRP} \mathrm{dilutions} \mathrm{were} \mathrm{made} \mathrm{in} \mathrm{1xPBS.} \mathrm{For} \mathrm{each}$ well, $2.0 \mu \mathrm{l}$ of HRP dilution, $1.0 \mu \mathrm{l}$ of $0.017 \mathrm{M}$ DMPA in DMF, and $1.0 \mu \mathrm{l}$ of 1 step ABTS was pipetted. For experiments testing the order of addition, the DMPA/DMF solution was pipetted onto the well and allowed to dry for 5 minutes before the addition of HRP and ABTS. The device was allowed to dry for 30 minutes before the device was scanned and analyzed in ImageJ. Each dilution was tested in 10 replicates.

\subsubsection{UV tolerance of HRP via solution deposition}

UV tolerance of HRP was tested with $20,10,5,3,1,0.5$, and $0.1 \mathrm{U} / \mathrm{ml}$ serial dilutions on a paper based 96 well plate. For each well, $2.0 \mu \mathrm{l}$ of an HRP dilution was deposited and exposed to UV irradiation at $365 \mathrm{~nm}$ for $10,30,45$, or 60 seconds. $1.0 \mu \mathrm{l}$ of 1 step ABTS was then pipetted and the device was allowed to dry for 30 minutes before scanning and analyzing the device in ImageJ. Each dilution was tested in 10 replicates.

\subsubsection{DMPA/DMF and UV tolerance of HRP via solution deposition}

The tolerance of serially diluted HRP solutions was tested on a paper based 96 well plate. 20, 10, 5, 3, 1, 0.5, and $0.1 \mathrm{U} / \mathrm{ml} \mathrm{HRP}$ dilutions were made in 1xPBS. For each well, $1.0 \mu \mathrm{l}$ of $0.017 \mathrm{M}$ DMPA in DMF was pipetted and allowed to dry for 5 minutes. Then, 
$2.0 \mu \mathrm{l}$ of an HRP dilution was added and exposed to UV irradiation at $365 \mathrm{~nm}$ for 10 or 30 seconds. Finally, $1.0 \mu \mathrm{l}$ of 1 step ABTS was pipetted. The device was allowed to dry for 30 minutes before the device was scanned and analyzed in ImageJ. Each dilution was tested in 10 replicates.

3.5.6. A novel microPAD for HRP immobilization via thiolene click chemistry

The microPAD design shown in Figure $\mathbf{4 8}$ was wax printed onto an alkene functionalized sheet of chromatography paper. For each device, $1.5 \mu \mathrm{l}$ of $0.017 \mathrm{M}$ DMPA in DMF was pipetted onto the experimental zone and allowed to dry for five minutes. $2 \mu \mathrm{l}$ of $20 \mathrm{U} / \mathrm{mL}$ HRP was pipetted onto the experimental zone and exposed to UV irradiation at $365 \mathrm{~nm}$ for 10 seconds. $32 \mu$ of deionized water was then added to the wash zone to clear out any unbound HRP. Finally, $1 \mu \mathrm{l}$ of 1 step ABTS was pipetted into the experimental, control, and waste zone. The device was allowed to dry for 30 minutes before scanning and analysis in ImageJ software.

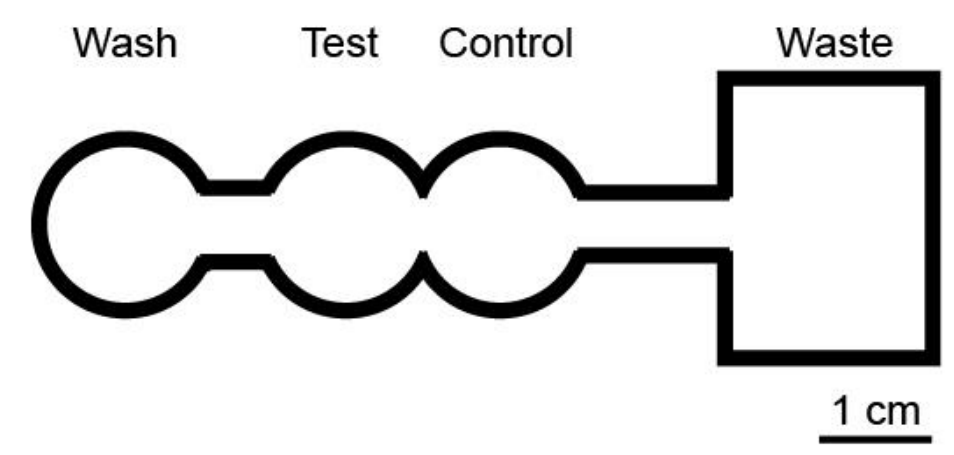

Figure 48. Novel microPAD design for HRP immobilization via thiol-ene click chemistry. 


\section{CHAPTER 4. CONCLUSIONS AND FUTURE WORK}

Reagent pencils have great potential in creating a new platform for low-cost diagnostic assays. In addition to their simple fabrication process and long shelf life, reagent pencils are versatile and can be customized in reagent type, polymer composition, etc. By incorporating enzymes in a solid state, and thereby extending the shelf life of the enzyme itself, the reagent pencil has the capacity to be an easy-access, high throughput diagnostic assay for POC and consumer friendly applications.

Initially, the characterization of the polymer component of reagent pencils was studied. In particular, it was found that lower molecular weight PEG components created a softer reagent pencil and higher molecular weight PEG created a harder, more brittle reagent pencil. These characteristics were quantified with wear resistance testing. Composites of reagent pencils with PEG components of different molecular weights reflected wear that is about the average of the wear for reagent pencils with singular, noncomposite PEG compositions. Further, the effects of PEG chain end functionality on reagent pencil performance was also investigated with PEGME and PEGdiME reagent pencils, respectively.

The type of reagent incorporated into the reagent pencil was also investigated. For this work, a blue dye, glucose, and HRP were incorporated at different $w / w \%$ with varying 
polymer composition. Utilizing the wear test and release profile of each respective reagent type, the reagent release efficiency was determined.

In a related study, the effects of PEG molecular weight and concentration on enzyme stability was investigated. To investigate this effect thoroughly, both HRP and ALP activity in the presence of PEG was tested. For HRP, it was found that PEG at any molecular weight above $400 \mathrm{~g} / \mathrm{mol}$ has the capacity to stabilize and improve the activity of HRP, regardless of the polymer concentration. At molecular weights below $400 \mathrm{~g} / \mathrm{mol}$, PEG failed to improve HRP activity and had no effect, even at higher polymer concentrations. For ALP, there also appears to be a molecular weight dependence on improving the enzyme activity at lower enzyme concentrations. In this case, however, the minimum molecular weight threshold has not been determined and can be studied as part of future work.

In an effort to develop a stimuli responsive component of microPADs, the manipulation PNIPAM and its LCST was investigated in the context of reagent pencils. In particular, PNIPAM was chosen for its potential to manipulate fluid flow through microPADs based on its responsiveness to salt solutions. It was found that a $1 \mathrm{M} \mathrm{Na}_{2} \mathrm{SO}_{4}$ worked best at lowering the LCST of PNIPAM. Two different molecular weights of PNIPAM were investigated for their effects on vertical and lateral flow microPADs. In both cases, a molecular weight and concentration dependence of PNIPAM affected the ability 
of PNIPAM to block fluid flow. Because PNIPAM is soluble in water, this effect becomes limited when $\mathrm{DI} \mathrm{H}_{2} \mathrm{O}$ solutions are washed through the device prior to the delivery of salt solutions. Thus, thiol-ene click chemistry was successfully employed to covalently bind PNIPAM onto alkene modified chromatography paper and retain $\mathrm{DI} \mathrm{H}_{2} \mathrm{O}$ wash tolerance. In this way, PNIPAM can be incorporated into reagent pencils as a novel, salt responsive gate that can aid more complex and sophisticated microPAD design.

In the future, other click chemistry based microPAD assays can also be developed. The condition tolerance of HRP was also investigated in preparation for thiolene click chemistry onto modified chromatography paper. It was found that the activity of HRP at different concentrations diminished after $\geq 10$ seconds of UV irradiation. Future studies to improve HRP tolerance to UV exposure can include studying UV irradiation of HRP in the presence of PEG, as it has already been shown to improve the activity of HRP in this work. If successful, it may be possible to utilize click chemistry for enzymes with free cysteine moieties whose covalent binding will not interfere with the activity of the enzyme's active site. The lateral and vertical wash response profiles for HRP and BD were also investigated in order to determine the necessity of covalent modification onto the paper substrate. It was found that, for both lateral and vertical tests, HRP responded with a high wash tolerance and did not call for covalent modification. The response profile of $\mathrm{BD}$, however, was more representative of a water soluble reagent that has low wash tolerance. In the case of a reagent with a similar response profile, future work can 
investigate the use of click chemistry to bind these reagents to the paper substrate to improve their wash tolerance.

A novel microPAD was designed to demonstrate enzyme grafting onto an alkenemodified chromatography paper substrate, as shown in Figure 48. On this device, HRP can be conjugated onto the test zone in the presence of DMPA. The control zone would exhibit HRP without the presence of the photoinitator. Thus, upon UV irradiation, HRP on the test zone would become conjugated onto the substrate whereas HRP in the control zone would be not be. The conjugated HRP would be expected to retain its functionality even after several $\mathrm{DI} \mathrm{H}_{2} \mathrm{O}$ washes, as demonstrated by the work done with PNIPAM reagent pencils. Immobilizing HRP, or other enzymes, would have great implications and potential in refining immunochemistry based fluorescent labeling, and would eliminate the blocking step commonly seen in other protein immobilization techniques.

Overall, the utility of reagent pencils has been shown to be versatile in the reinforcement of enzyme stability, the introduction of novel stimuli responsive tools for microPADs, and potential alternative reagent immobilization techniques. In the future, reagent pencils and microPADs are seen as a rapid, quantitative diagnostic tool that can be customized at a POC location easily based on the tools developed from this work. 


\section{REFERENCES}

1. York N, Press A. Analytical chemistry (114). 1949;48(85).

2. Mu X, Zhang L, Chang S, Cui W, Zheng Z. Multiplex microfluidic paper-based immunoassay for the diagnosis of hepatitis $\mathrm{C}$ virus infection. Anal Chem. 2014;86(11):5338-5344. doi:10.1021/ac500247f.

3. Park TS, Harshman DK, Fronczek CF, Yoon J. Smartphone Detection of Escherichia Coli From Wastewater Utilizing Paper Microfluidics. 2013;15(October):1347-1349.

4. Ryan P, Zabetakis D, Stenger D, Trammell S. Integrating Paper Chromatography with Electrochemical Detection for the Trace Analysis of TNT in Soil. Sensors. 2015;15(7):17048-17056. doi:10.3390/s150717048.

5. Dungchai W, Chailapakul O, Henry CS. Electrochemical detection for paper-based microfluidics. Anal Chem. 2009;81(14):5821-5826. doi:10.1021/ac9007573.

6. Mentele MM, Cunningham J, Koehler K, Volckens J, Henry CS. Microfluidic paperbased analytical device for particulate metals. Anal Chem. 2012;84(10):4474-4480. doi:10.1021/ac300309c.

7. Chen X, Chen J, Wang F, et al. Determination of glucose and uric acid with bienzyme colorimetry on microfluidic paper-based analysis devices. Biosens Bioelectron. 2012;35(1):363-368. doi:10.1016/j.bios.2012.03.018.

8. Whitesides GM. The origins and the future of microfluidics. Nature. 2006;442(7101):368-373. doi:10.1038/nature05058.

9. Mace CR, Deraney RN. Manufacturing prototypes for paper-based diagnostic devices. Microfluid Nanofluidics. 2014;16(5):801-809. doi:10.1007/s10404-0131314-6.

10. Lisowski P, Zarzycki PK. Microfluidic paper-based analytical devices ( $\mu$ PADs) and micro total analysis systems ( $\mu$ TAS): Development, applications and future trends. Chromatographia. 2013;76(19-20):1201-1214. doi:10.1007/s10337-013-2413-y.

11. Martinez AW, Phillips ST, Whitesides GM, Carrilho E. Diagnostics for the developing world: Microfluidic paper-based analytical devices. Anal Chem. 2010;82(1):3-10.

12. Khan MS, Thouas G, Shen W, Whyte G, Garnier G. Paper diagnostic for instantaneous blood typing. Anal Chem. 2010;82(10):4158-4164. doi:10.1021/ac100341n.

13. Liu H, Crooks RM. Three-dimensional paper microfluidic devices assembled using 
the principles of origami. J Am Chem Soc. 2011;133(44):17564-17566. doi:10.1021/ja2071779.

14. Tao J, Ma W, Liu N, et al. High-Performance Solid-State Supercapacitors Fabricated by Pencil Drawing and Polypyrrole Depositing on Paper Substrate. Nano-Micro Lett. 2015;7(3):276-281. doi:10.1007/s40820-015-0039-3.

15. Gimenez A. ZnO- Paper Based Photoconductive UV Sensor. J Phys .... 2010;2(3):282-287. http://pubs.acs.org/doi/abs/10.1021/jp107812w.

16. Dey R, Kar S, Joshi S, Maiti TK, Chakraborty S. Ultra-low-cost "paper-and-pencil" device for electrically controlled micromixing of analytes. Microfluid Nanofluidics. 2015;19(2):375-383. doi:10.1007/s10404-015-1567-3.

17. Krainer FW, Glieder A. An updated view on horseradish peroxidases: recombinant production and biotechnological applications. Appl Microbiol Biotechnol. 2015;99(4):1611-1625. doi:10.1007/s00253-014-6346-7.

18. O'Brien AM, Ó'Fágáin C, Nielsen PF, Welinder KG. Location of crosslinks in chemically stabilized horseradish peroxidase: Implications for design of crosslinks. Biotechnol Bioeng. 2001;76(4):277-284. doi:10.1002/bit.1194.

19. Juarez-Moreno K, Ayala M, Vazquez-Duhalt R. Antioxidant Capacity of Poly(Ethylene Glycol) (PEG) as Protection Mechanism Against Hydrogen Peroxide Inactivation of Peroxidases. Appl Biochem Biotechnol. 2015;177(6):1364-1373. doi:10.1007/s12010-015-1820-y.

20. Schmaljohann D. Thermo- and $\mathrm{pH}$-responsive polymers in drug delivery. Adv Drug Deliv Rev. 2006;58(15):1655-1670. doi:10.1016/j.addr.2006.09.020.

21. Eeckman F, Moës AJ, Amighi K. Evaluation of a new controlled-drug delivery concept based on the use of thermoresponsive polymers. Int $\mathrm{J}$ Pharm. 2002;241(1):113-125. doi:10.1016/S0378-5173(02)00198-9.

22. Roth PJ, Jochum FD, Theato P. UCST-type behavior of poly[oligo(ethylene glycol) methyl ether methacrylate] (POEGMA) in aliphatic alcohols: solvent, co-solvent, molecular weight, and end group dependences. Soft Matter. 2011;7(6):2484 . doi:10.1039/c0sm01324b.

23. Du H, Wickramasinghe $\mathrm{R}$, Qian X. Effects of salt on the lower critical solution temperature of poly (N-isopropylacrylamide). J Phys Chem B. 2010;114(49):1659416604. doi:10.1021/jp105652c.

24. Rimmer S, Soutar I, Swanson L. Switching the conformational behaviour of poly(Nisopropyl acrylamide). Polym Int. 2009;58(3):273-278. doi:10.1002/pi.2537.

25. Xi W, Scott TF, Kloxin CJ, Bowman CN. Click chemistry in materials science. Adv 
Funct Mater. 2014;24(18):2572-2590. doi:10.1002/adfm.201302847. 